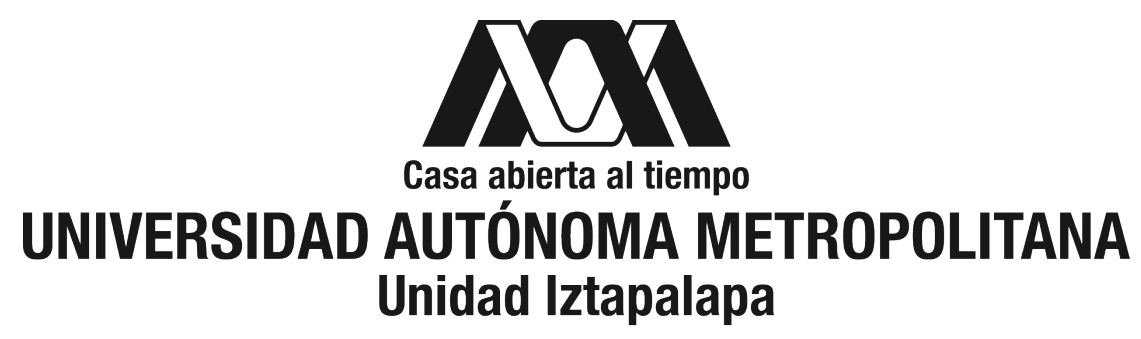

División de Ciencias Básicas e Ingeniería

\title{
Estudio teórico de cupratos de litio como posibles materiales catódicos para baterías
}

Tesis presentada para obtener el grado de:

Doctor en Ciencias (Química)

$$
\text { Prese n } \mathrm{ta} \text { : }
$$

M. Q. B. Luis Ignacio Perea Ramírez

Asesores:

Dr. Marcelo Enrique Galván Espinosa

Dr. Alfredo Augusto Guevara García

Sinodales:

Dr. Ignacio González Martínez

Dr. Joel Ireta Moreno

Dra. Annia Galano Jiménez

Dr. Mario Fidel García Sánchez

Dr. Joaquín Barroso Flores 

Dedicado a mi Padre Jehová, quien me sostiene con la siguiente promesa:

No temas, porque yo estoy contigo;

No te desalientes, porque yo soy tu Dios.

Te fortaleceré,

ciertamente te ayudaré,

Sí, te sostendré con la diestra de Mi Justicia.

Isaías 41:10

Y la mayor bendición que tengo, mi hijo Luis Alexander 



\section{Agradecimientos}

En primer lugar doy gracias a Jehová mi Padre, por su amor, misericordia y cada día de vida que me ha concedido. Siempre ha estado a mi lado y nunca me ha abandonado, por difícil que fuera la situación, siempre me mostró su amor y apoyo. Una de las bendiciones que Dios da al ser humano, es la familia, personas con las que no sólo compartes lazos de sangre, sino un conjunto de experiencias. La familia es el primer acercamiento a la convivencia con los demás. Por ello agradezco a toda mi familia por su constante ánimo y apoyo, en especial a mi hijo, quien es mi mayor bendición, la mayor prueba del amor que Dios me tiene. Gracias a mi Mamá por sus consejos, su tiempo y educarme con el ejemplo.

Agradezco a mis asesores, el Dr. Marcelo y el Dr. Alfredo, quienes me han apoyado enormemente. Con su paciencia y dedicación me dieron el ejemplo de cómo llevar a cabo un proyecto de investigación de este nivel y deseo poder ser a futuro un gran científico como ellos.

También agradezco a cada uno de los integrantes del área de Fisicoquímica Teórica, profesores y estudiantes, quienes siempre brindaron un ambiente solidario para trabajar y por lo seminarios impartidos, mostrando la variedad de temas y perspectivas del área. También agradezco al área de Electroquímica por los seminarios y discusiones generadas.

Finalmente agradezco a CONACYT por la beca de Doctorado No. 398738 y al apoyo 28546 con cargo al proyecto CB 2016/286013, así como al Laboratorio Nacional de Cómputo de Alto Desempeño (LANCAD) y CONACYT por el tiempo de cómputo proporcionado en la supercomputadora Yoltla, en el Laboratorio de Supercómputo y Visualización en Paralelo (LSVP) de la UAM-Iztapalapa. 



\section{Resumen}

En el presente trabajo, la densidad de estados proyectada (DOS-p) y la blandura local, $s(\mathbf{r})$, fueron estudiadas como propiedades relacionadas con el desempeño de $\mathrm{Li}_{2} \mathrm{CuO}_{2}, \mathrm{LiCuO}_{2}$ y $\mathrm{Li}_{2} \mathrm{Cu}_{1-x} \mathrm{M}_{x} \mathrm{O}_{2}$ ( $\mathrm{M}=\mathrm{Co}$, Ni o Ti, $x=0.25$ o 0.5 ) como materiales catódicos. Todos los cálculos fueron realizados en el marco de la teoría de los funcionales de la densidad (DFT por sus siglas en inglés) bajo condiciones periódicas. Mediante el análisis de la DOS-p de cada sistema, los estados electrónicos involucrados en el proceso inicial de carga de la batería (oxidación del cátodo) fueron identificados. Esta información permitió establecer la participación del oxígeno en el proceso redox, que ha sido relacionada con una disminución en la capacidad durante el proceso de carga-descarga. La utilización de la $s(\mathbf{r})$ ha sido propuesta en lugar de la DOS-p para analizar los procesos redox iniciales. Se encontró que es mejor usar la $s(\mathbf{r})$, debido a que muestra los sitios susceptibles a perder electrones, permitiendo hacer comparaciones directas entre sistemas, en una forma más simple que la DOS-p. Los resultados obtenidos predicen que los sistemas $\mathrm{Li}_{2} \mathrm{Cu}_{1-x} \mathrm{Ti}_{x} \mathrm{O}_{2}$ involucran menor participación redox de oxígeno y, por lo tanto, una mejor retención de la capacidad durante el proceso de carga-descarga. Además, se utilizó la $s(\mathbf{r})$ para identificar los átomos más susceptibles a oxidarse, en sulfuros de hierro de importancia hidrometalúrgica para México. Una buena concordancia fue encontrada entre los átomos susceptibles a oxidarse predichos por la $s(\mathbf{r})$ y aquellos determinados en estudios experimentales. Por lo tanto, demostramos que la $s(\mathbf{r})$ puede ser aplicada a diferentes procesos de redox en sólidos. 



\section{Abstract}

In this work, the projected density of states (DOS-p) and the local softness, $s(\mathbf{r})$, were studied as properties related with the performance of $\mathrm{Li}_{2} \mathrm{CuO}_{2}, \mathrm{LiCuO}_{2}$, and $\mathrm{Li}_{2} \mathrm{Cu}_{1-x} \mathrm{M}_{x} \mathrm{O}_{2}$ ( $\mathrm{M}=\mathrm{Co}, \mathrm{Ni}$ or Ti, $x=0.25$ or 0.5 ) as cathode materials. All calculations were done within the framework of the density functional theory (DFT) using periodic boundary conditions. Through the analysis of the DOS-p of each system, the electronic states involved in the initial charge process of the battery (cathode oxidation) were identified. This information allowed stablishing the oxygen participation in the redox process that has been related to a decrease in the capacity during the charge-discharge process. The use of the $s(\mathbf{r})$ has been proposed instead of DOS-p to analyze the initial redox processes. It was found that it is better to use the $s(\mathbf{r})$ because it shows susceptible sites to lose electrons and allow us to do direct comparisons between the systems in a simpler way than the DOS-p. The obtained results predict that $\mathrm{Li}_{2} \mathrm{Cu}_{1-x} \mathrm{Ti}_{x} \mathrm{O}_{2}$ systems involve less oxygen redox participation and therefore a better retention of capacity during the charge-discharge process. In addition, the $s(\mathbf{r})$ was used to identify the most susceptible atoms toward oxidation, in iron sulphides of hydrometallurgical importance for Mexico. A good agreement was found between the predicted susceptible atoms to oxidation by $s(\mathbf{r})$ and those determined in experimental studies. Hence, we have demonstrated that the $s(\mathbf{r})$ could be applied to different redox process in solids. 



\title{
CONTENIDO
}

Agradecimientos

Resumen

Abstract

Glosario

1 Introducción

2 Antecedentes

2.1 Generalidades de las baterías de ion litio . . . . . . . . . . . . . . . . . . 19

2.2 Interés por los cupratos de litio . . . . . . . . . . . . 20

2.2 .1 Reacciones químicas laterales . . . . . . . . . . . . 22

2.2 .2 Transformaciones estructurales . . . . . . . . . . . . 23

2.3 Sistemas derivados de $\mathrm{Li}_{2} \mathrm{CuO}_{2} \ldots \ldots \ldots \ldots \ldots \ldots$

2.4 Participación redox del oxígeno en cátodos basados en óxidos . . . . . . . 25

3 Marco Teórico 28

3.1 Ecuación de Schrödinger . . . . . . . . . . . . . . . . . . 28

3.1 .1 El principio variacional . . . . . . . . . . . . . . . 30

3.1 .2 Interacciones electrón-electrón $\ldots \ldots \ldots \ldots \ldots$

3.2 Teoría de los funcionales de la densidad . . . . . . . . . . . . . 33

3.2.1 Los teoremas de Hohenberg y Kohn . . . . . . . . . . . . . . . . 33

3.2 .2 La ecuación de Kohn-Sham (KS) . . . . . . . . . . . . . . . 34

3.3 Funcionales de intercambio-correlación . . . . . . . . . . . . . . . . 37

3.3.1 Aproximación de la densidad local . . . . . . . . . . . . . 37

3.3.2 Aproximación del gradiente generalizado . . . . . . . . . 38

3.3.3 Funcionales con correcciones tipo Hubbard . . . . . . . . . . . . . 39

3.3.4 Aproximación del funcional de rango separado HSE06 . . . . . . . 41

\section{5}

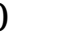


3.4 Estudio de la estructura electrónica de sólidos . . . . . . . . . . . . . . . 42

3.4 .1 Estructura cristalina . . . . . . . . . . . . . . . . . . 42

3.4 .2 Teorema de Bloch . . . . . . . . . . . . . . . 43

3.4.3 Método proyector de ondas aumentadas . . . . . . . . . . . . 44

4 Hipótesis $\quad 50$

5 Objetivos 51

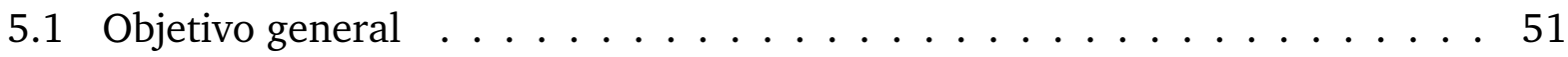

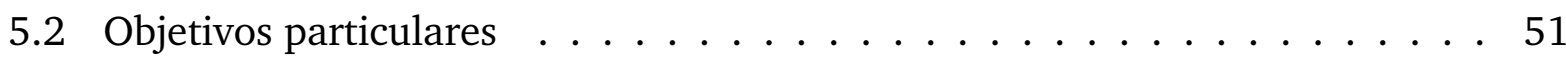

6 Metodología $\mathbf{5 2}$

6.1 Sistemas $\mathrm{Li}_{2} \mathrm{CuO}_{2} \mathrm{y} \mathrm{LiCuO}_{2} \ldots \ldots \ldots \ldots \ldots \ldots \ldots$

6.2 Sistemas del tipo $\mathrm{Li}_{2} \mathrm{Cu}_{0.5} \mathrm{M}_{0.5} \mathrm{O}_{2} \ldots \ldots \ldots \ldots \ldots \ldots \ldots \ldots \ldots \ldots$

6.3 Cálculo de la densidad de estados proyectada y la blandura local . . . . . . 54

6.4 Cálculo del voltaje teórico promedio de inserción de litio . . . . . . . . . . 57

6.5 Sistemas del tipo $\mathrm{Li}_{2} \mathrm{Cu}_{0.75} \mathrm{M}_{0.25} \mathrm{O}_{2} \ldots \ldots \ldots \ldots \ldots$

7 Resultados $\quad 61$

7.1 Parámetros estructurales de $\mathrm{LiCuO}_{2} \mathrm{y} \mathrm{Li}_{2} \mathrm{CuO}_{2} \ldots \ldots \ldots \ldots$

7.2 Datos estructurales de $\mathrm{Li}_{2} \mathrm{Cu}_{0.5} \mathrm{M}_{0.5} \mathrm{O}_{2}$ obtenidos con $\mathrm{PBE}+\mathrm{U} \ldots \ldots 2$

7.2.1 Parámetros estructurales . . . . . . . . . . . . . . 62

7.2.2 Distancias de enlace . . . . . . . . . . . . . . . . . . . 64

7.3 Estado de magnetización obtenido para cada sistema con el funcional HSE06 66

7.4 Análisis de la densidad de estados proyectada . . . . . . . . . . . . 68

7.5 Análisis de la blandura local . . . . . . . . . . . . . . . . . . . 74

$7.5 .1 \mathrm{Li}_{2} \mathrm{CuO}_{2} \mathrm{y} \mathrm{LiCuO}_{2} \ldots \ldots \ldots \ldots \ldots \ldots$

7.5.2 Sistemas del tipo $\mathrm{Li}_{2} \mathrm{Cu}_{0.5} \mathrm{M}_{0.5} \mathrm{O}_{2} \ldots \ldots \ldots \ldots \ldots \ldots$

7.6 Blandura local con otros funcionales de intercambio-correlación . . . . . . . 77

7.6.1 Sistemas $\mathrm{Li}_{2} \mathrm{CuO}_{2} \mathrm{y} \mathrm{LiCuO}_{2} \ldots \ldots \ldots \ldots \ldots \ldots$

7.6.2 Sistemas del tipo $\mathrm{Li}_{2} \mathrm{Cu}_{0.5} \mathrm{M}_{0.5} \mathrm{O}_{2} \ldots \ldots \ldots \ldots \ldots \ldots$

7.7 Voltaje de inserción de litio en $\mathrm{LiCuO}_{2}, \mathrm{Li}_{2} \mathrm{CuO}_{2} \mathrm{y} \mathrm{Li}_{2} \mathrm{Cu}_{0.5} \mathrm{M}_{0.5} \mathrm{O}_{2} \ldots \ldots$. . 82

7.8 Sistemas del tipo $\mathrm{Li}_{2} \mathrm{Cu}_{0.75} \mathrm{M}_{0.25} \mathrm{O}_{2} \ldots \ldots \ldots \ldots \ldots \ldots$

7.8.1 Densidad de estados proyectada de los sistemas $\mathrm{Li}_{2} \mathrm{Cu}_{0.75} \mathrm{M}_{0.25} \mathrm{O}_{2} \quad$. $\quad 82$

7.8.2 Blandura local de los sistemas $\mathrm{Li}_{2} \mathrm{Cu}_{0.75} \mathrm{M}_{0.25} \mathrm{O}_{2} \ldots \ldots \ldots \ldots$

8 Conclusiones y Perspectivas $\quad 87$

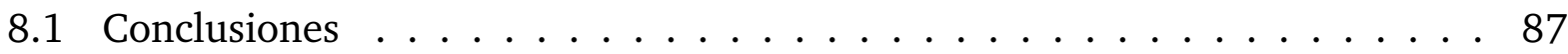

8.2 Perspectivas . . . . . . . . . . . . . . . . . . 89 
9 Apéndices $\quad \mathbf{9 0}$

9.1 Definiciones de los parámetros de desempeño de las baterías de ion litio . 90

9.2 Análisis de la densidad de estados proyectada y la blandura local en minerales 91

9.2 .1 Calcopirita . . . . . . . . . . . . . . . . . 92

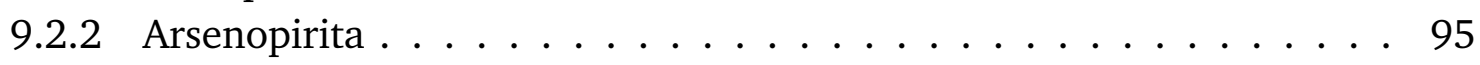

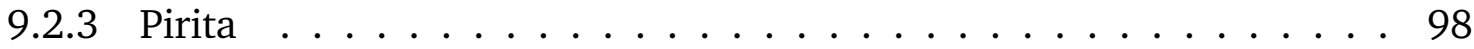

9.2 .4 Marcasita . . . . . . . . . . . . . . . . . . 101

9.3 Artículo . . . . . . . . . . . . . . . . . . . 104

$\begin{array}{ll}\text { Referencias. } & 113\end{array}$ 



\section{GlosarIO}

$\begin{array}{ll}\text { BIL } & \text { Batería de ion litio } \\ \text { CUP } & \text { Celda unitaria primitiva } \\ \text { DOS } & \text { Densidad de estados } \\ \text { DOS-p } & \text { Densidad de estados proyectada } \\ \text { DFT } & \text { Teoría de los funcionales de la densidad } \\ \text { EA } & \text { Esfera de aumento } \\ \text { GGA } & \text { Aproximación del gradiente generalizado } \\ \text { GGA+U } & \text { Aproximación del gradiente generalizado con correcciones tipo Hubbard } \\ \text { HF } & \text { Hartree-Fock } \\ \text { HK } & \text { Hohenberg y Kohn } \\ \text { ICSD } & \text { Inorganic Crystal Structure Database } \\ \text { KS } & \text { Kohn-Sham } \\ \text { LDA } & \text { Aproximación de la densidad local } \\ \text { LSDA } & \text { Aproximación de la densidad local de espín } \\ \text { OPW } & \text { Ondas planas ortogonalizadas } \\ \text { PAW } & \text { Proyector de ondas aumentado } \\ \text { PS } & \text { Pseudopotencial } \\ \text { TCC } & \text { Teoría del campo cristalino } \\ \text { VASP } & \text { Vienna Ab initio Simulation Package } \\ \text { VESTA } & \text { Visualization for Electronic and STructural Analysis } \\ \text { XC } & \text { Intercambio y correlación }\end{array}$





\section{Capítulo}

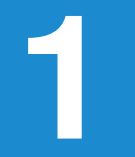

\section{INTRODUCCIÓN}

F la actualidad existe la necesidad de cubrir la demanda de energía eléctrica en forma costo, beneficio e impacto ambiental, ya que son aptas para el transporte y almacenamiento de energía eléctrica a partir de fuentes renovables, como la energía solar o la eólica. Para extender su uso más allá de los dispositivos móviles, es necesario que posean una mayor densidad de energía, reflejado, por ejemplo, en una mayor capacidad de carga-descarga por parte del material catódico de la batería. Por ello, es necesario generar nuevos materiales catódicos para la construcción de baterías recargables de ion litio, que posean una mayor capacidad de carga-descarga.

Este objetivo demanda un trabajo conjunto, teórico-experimental, que permita ahorrar tiempo y recursos para dirigir los estudios hacia los candidatos más prometedores. En este aspecto, los estudios teóricos proporcionan una herramienta que permite, con un cierto criterio (por ejemplo, el voltaje de inserción de litio) hacer esta selección y cuentan con la ventaja, si así se requiere, de poder restringir el estudio al cambio de una sola variable manteniendo las demás constantes.

El material catódico puede estar constituido por óxidos de metales de transición, susceptibles a liberar $\mathrm{O}_{2}$ durante el proceso de carga, lo cual disminuye su rendimiento y representa un riesgo en cuestión de seguridad. De la liberación de $\mathrm{O}_{2}$ se infiere que el proceso redox de este tipo de materiales catódicos, no radica exclusivamente en el metal de transición, sino también en los átomos de oxígeno presentes en la red del material, tal como en el caso del $\mathrm{Li}_{2} \mathrm{CuO}_{2}$.

En el proceso de carga, la participación redox de oxígeno en $\mathrm{Li}_{2} \mathrm{CuO}_{2}$, ha sido relacionada con: 1) el cambio de la densidad electrónica después de la sustracción de carga y 2) la contribución de oxígeno en los estados cercanos al nivel de Fermi. Se ha mostrado que al retirar un electrón de $\mathrm{Li}_{2} \mathrm{CuO}_{2}$ y restar las densidades electrónicas, los sitios afectados corresponden al plano cuadrado de $\mathrm{CuO}_{4}$. Por otro lado, el análisis de la densidad de estados proyectada (projected density of states, DOS-p) de $\mathrm{Li}_{2} \mathrm{CuO}_{2}$ y LiCuO estado desocupado por la deslitiación corresponde en mayor medida a oxígeno, pero no es 
sencillo hacer una comparación relativa directa.

La contribución más importante del presente trabajo fue utilizar la blandura local, $s(\mathbf{r})$, como alternativa a la DOS-p, para el análisis del proceso de oxidación en $\mathrm{Li}_{2} \mathrm{CuO}_{2}$ y sistemas derivados $\mathrm{Li}_{2} \mathrm{Cu}_{1-x} \mathrm{M}_{x} \mathrm{O}_{2}$. Como se mencionará más adelante, fue posible realizar comparaciones directas entre diferentes sistemas, de forma más práctica que la DOS-p. En cada sistema, se analizó la susceptibilidad de los átomos de oxígeno a ser oxidados durante el proceso de carga, es decir, la participación redox de los átomos de oxígeno del sistema, todo esto con el fin de proponer la generación de nuevos ambientes químicos, $\mathrm{M}-\mathrm{O}$ (M $=\mathrm{Co}, \mathrm{Ni}$ o Ti), en la estructura del $\mathrm{Li}_{2} \mathrm{CuO}_{2}$ para mejorar la retención de su capacidad durante los procesos de carga-descarga, donde la regulación de la participación redox de los oxígenos del sistema, resulta de gran importancia para este fin. 


\section{Capítulo \\ 2 \\ ANTECEDENTES}

\subsection{Generalidades de las baterías de ion litio}

Una batería de ion litio (BIL) es un tipo de sistema de almacenamiento de energía [1, 2], construido a través de celdas de ion litio conectadas en paralelo, serie o configuraciones combinadas [3]. Cada una de estas celdas (Figura 2-1) consiste básicamente de un cáto$\mathrm{do}^{\mathrm{a}} \mathrm{y}$ un ánodo ${ }^{\mathrm{b}}$ inmersos en un electrolito. Los electrodos se encuentran separados por una membrana de polímero microporosa, que permite el intercambio de iones litio entre los dos electrodos $[1,3,4]$, pero no de electrones $[3,5]$.

El funcionamiento de la celda de ion litio es descrito a través de un mecanismo conocido como shuttle chair, donde los iones litio son transportados entre el cátodo y el ánodo durante los ciclos de carga y descarga. Durante el proceso de carga, los dos electrodos son conectados a una fuente de alimentación eléctrica externa. Los electrones son forzados a liberarse del cátodo y moverse, a través de un circuito externo, hacia el ánodo [3]. Simultáneamente los iones litio se mueven en la misma dirección dentro del electrolito $[3,5,6]$. Durante el proceso de descarga sucede el proceso inverso, la diferencia de potencial electroquímico de litio entre el ánodo y cátodo, obliga a los iones litio a moverse del ánodo al cátodo a través del electrolito así como los electrones en el mismo sentido a través del circuito externo, para realizar trabajo $[3,4,7]$. De esta manera, la energía externa está electroquímicamente almacenada en la batería en forma de energía química en los materiales anódicos y catódicos aprovechando las diferencias de los potenciales químicos del litio entre los dos electrodos [3,7].

En las condiciones de operación de las baterías, debido a las transferencias de carga y transporte de masa, es posible que sucedan cambios estructurales y transiciones de fase que, en conjunto impactan en los parámetros de desempeño de la BIL [2], tales como:

${ }^{a}$ Electrodo en el que toma lugar la reducción.

bElectrodo en el que toma lugar la oxidación. 


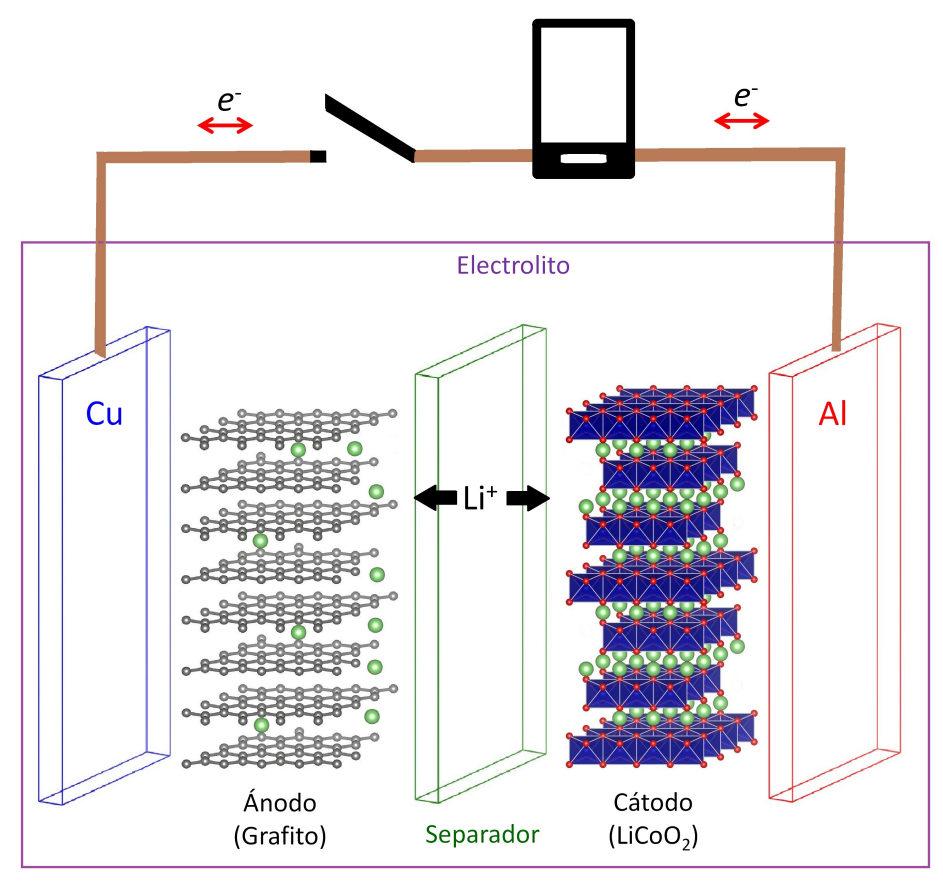

Figura 2-1: Ilustración de los componentes básicos de una celda de ion litio.

1) energía específica, 2) energía volumétrica, 3) capacidad específica, 4) ciclabilidad, 5) seguridad, 6) tolerancia de abuso y 7) velocidad de carga-descarga [3] (cada uno definido en el apéndice 9.1).

\subsection{Interés por los cupratos de litio}

Los materiales utilizados para la construcción de cátodos de una BIL se pueden agrupar en [2]:

1. Óxidos (por ejemplo, $\mathrm{LiCoO}_{2}, \mathrm{LiMn}_{2} \mathrm{O}_{4} \mathrm{y} \mathrm{Li}{ }_{2} \mathrm{CuO}_{2}$ ) y

2. Polioxoaniones (p. ej. $\mathrm{LiFePO}_{4}$ y $\mathrm{LiFeSO}_{4} \mathrm{~F}$ ),

todos estos, a pesar de poseer aplicaciones prácticas, no están exentos de limitaciones (Tabla 2-1). Dentro de las características deseables de un material catódico están: 1) mantener una alta capacidad después de repetidos ciclos de carga y descarga $\left.{ }^{c}, 2\right)$ ser seguro, 3) económico y 4) benigno con el medio ambiente ${ }^{\mathrm{d}}$ [8]. El cobre es un metal de transición abundante, relativamente económico y de menor toxicidad que cobalto y níquel [9].

\footnotetext{
${ }^{\mathrm{c}}$ Alrededor de miles de ciclos

${ }^{\mathrm{d}}$ Es decir, contar con la capacidad de ser reciclados.
} 
Como óxido litiado presenta las cualidades de ser de bajo costo y menos dañino al medio ambiente, en comparación a los sistemas basados en cobalto, por lo que ha sido de interés su estudio como material catódico, tal como es el caso del $\mathrm{Li}_{2} \mathrm{CuO}_{2}$ [8].

Tabla 2-1: Desventajas de algunos materiales catódicos

\begin{tabular}{|c|c|}
\hline Sistema & Desventajas \\
\hline $\mathrm{LiCoO}_{2}$ & $\begin{array}{l}\text { Tiende a colapsar tras una desintercalación de } \mathrm{Li}^{+} \text {mayor al } 50 \\
\text { y puede llevar a la liberación de oxígeno [10]. }\end{array}$ \\
\hline $\mathbf{L i M n}_{2} \mathbf{O}_{4}$ & $\begin{array}{l}\text { Disolución de } \mathrm{Mn}^{2+} \text { en la interfase con el electrolito, ocasionando } \\
\text { una disminución en su capacidad [11]. }\end{array}$ \\
\hline $\mathrm{LiFePO}_{4}$ & $\begin{array}{l}\left.\text { Capacidad"relativamente baja (100-110 } \mathrm{mA} \mathrm{h}^{-1}\right) \text { [12] en com- } \\
\text { paración a } \mathrm{LiCoO}_{2}\left(160 \mathrm{~mA} \mathrm{~h} \mathrm{~g}^{-1}\right) \text { [13]. }\end{array}$ \\
\hline $\mathrm{LiFeSO}_{4} \mathrm{~F}$ & $\begin{array}{l}\left.\text { Capacidad"relativamente baja (140 } \mathrm{mA} \mathrm{h}^{-1}\right) \text { [14] en compara- } \\
\text { ción a } \mathrm{LiCoO}_{2}\left(160 \mathrm{~mA} \mathrm{~h} \mathrm{\textrm {g } ^ { - 1 } )}[13] \text {. }\right.\end{array}$ \\
\hline
\end{tabular}

* En un material para electrodo, es la cantidad de carga eléctrica extraíble por unidad de masa [15].

$\mathrm{Li}_{2} \mathrm{CuO}_{2}$ es un óxido laminar no tóxico y de bajo costo (comparado a sistemas de cobalto) [8]. Cristaliza en una celda unitaria ortorrómbica de grupo espacial Immm [16], cada átomo de cobre está coordinado a cuatro átomos de oxígeno y forman planos cuadrados que comparten borde $[16,17]$, cada átomo de litio está coordinado a cuatro átomos de oxígeno y forman tetraedros que comparten borde [16](Figura 2-2). $\mathrm{Li}_{2} \mathrm{CuO}_{2}$ presenta un ordenamiento antiferromagnético [17].

Este material ha llamado la atención debido a que, teóricamente, puede intercalar dos $\mathrm{Li}^{+}$por unidad fórmula, que supera el límite práctico por unidad fórmula de materiales comerciales, (por ejemplo, $0.5 \mathrm{Li}^{+}$para $\mathrm{LiCoO}_{2}$ y $1 \mathrm{Li}^{+}$para $\mathrm{LiFePO}_{4}$ ) [8]. Este hecho le confiere una capacidad teórica relativamente alta de 245 y $489 \mathrm{~mA} \mathrm{~h} \mathrm{~g}^{-1}$ al intercalar uno y dos $\mathrm{Li}^{+}$, respectivamente [18]. Sin embargo, experimentalmente, ha mostrado poseer una gran disminución de la capacidad después del ciclo inicial de carga-descarga $(\sim 230$ $\mathrm{mA} \mathrm{h} / \mathrm{g}$ en la primera carga y $\sim 100 \mathrm{~mA} \mathrm{~h} / \mathrm{g}$ en la primera descarga) [8], lo que puede deberse a:

1. Reacciones químicas laterales en las cuales es posible que se libere oxígeno $[8,19]$,

2. Transformaciones estructurales que pueden llevar a la generación de otras fases [8, 16] y 


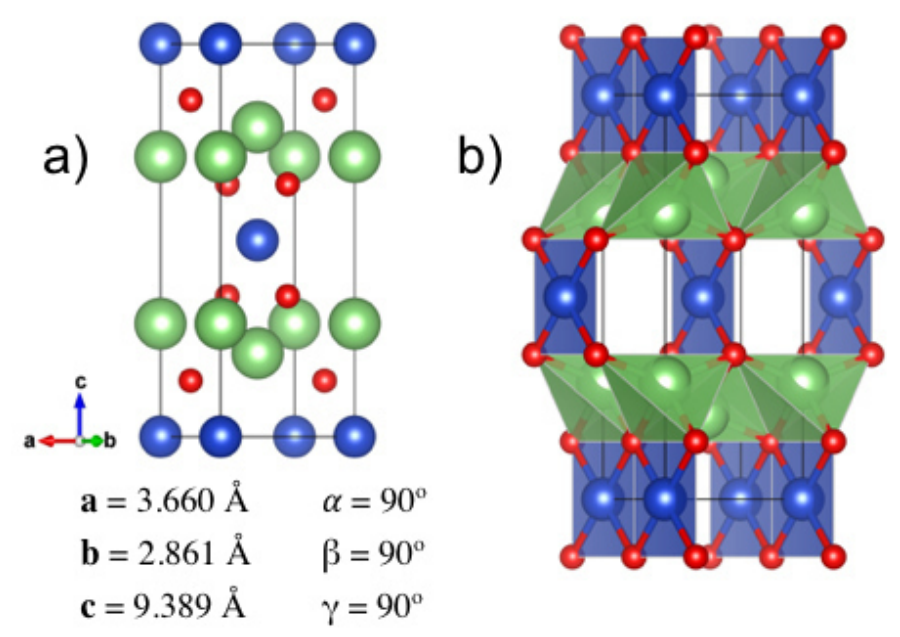

Figura 2-2: a) Celda unitaria de $\mathrm{Li}_{2} \mathrm{CuO}_{2}$ y b) coordinación de cada átomo dentro de la estructura cristalina de $\mathrm{Li}_{2} \mathrm{CuO}_{2}$. Las esferas color verde representan los átomos de litio, azules a los átomos de cobre y rojas a los átomos de oxígeno. Los parámetros de la celda unitaria fueron tomados de la referencia [16]

3. Otras: Método de síntesis, ensamble del cátodo así como la ventana de voltaje de trabajo [18]

La contribución de los dos primeros factores se explica a continuación.

\subsubsection{Reacciones químicas laterales}

El proceso ideal de carga y descarga en $\mathrm{Li}_{2} \mathrm{CuO}_{2}$ puede ser descrito por las ecuaciones (2-1) y (2-3) respectivamente, donde la desinserción e inserción de $\mathrm{Li}^{+}$es uniforme en todo el sistema, y por ello se espera la formación de una sola fase parcialmente deslitiada en la etapa de carga, $\mathrm{Li}_{2-x} \mathrm{CuO}$, la cual, durante la descarga, es litiada para recuperar la fase inicial $\left(\mathrm{Li}_{2} \mathrm{CuO}_{2}\right)$. Sin embargo, una vez que ocurre (2-1) es posible que parte de $\mathrm{Li}_{2-x} \mathrm{CuO}_{2}$ se continúe deslitiando para formar $\mathrm{CuO}$ y liberando $\mathrm{O}_{2}$ por medio de la reacción en (2-2). Esto trae como consecuencia que durante la descarga (litiación ) se genere un sistema multifase de composición $\mathrm{Li}_{2} \mathrm{CuO}_{2}$ vía (2-3) y $\mathrm{Li}_{x} \mathrm{CuO}_{2}$ vía (2-4) [8].

$$
\begin{gathered}
\mathrm{Li}_{2} \mathrm{CuO}_{2} \longrightarrow \mathrm{Li}_{2-x} \mathrm{CuO}_{2}+x \mathrm{Li}^{+}+x e^{-} \\
2 \mathrm{Li}_{2-x-y} \mathrm{CuO}_{2} \longrightarrow 2 \mathrm{CuO}+2 y \mathrm{Li}^{+}+\mathrm{O}_{2}+2 y e^{-}(x+y \approx 1)
\end{gathered}
$$




$$
\begin{gathered}
\mathrm{Li}_{2-x} \mathrm{CuO}_{2}+x \mathrm{Li}^{+}+x e^{-} \longrightarrow \mathrm{Li}_{2} \mathrm{CuO}_{2} \\
2 \mathrm{CuO}+2 x \mathrm{Li}^{+}+\mathrm{O}_{2}+2 x e^{-} \longrightarrow 2 \mathrm{Li}_{x} \mathrm{CuO}_{2} \\
\mathrm{Li}_{x} \mathrm{CuO}_{y} \text { puede ser } \mathrm{LiCuO}_{2} \text { o } \mathrm{Li}_{1.5} \mathrm{CuO}_{2}
\end{gathered}
$$

\subsubsection{Transformaciones estructurales}

Durante el proceso de carga (deslitiación) es posible formar fases como $\mathrm{Li}_{1.5} \mathrm{CuO}_{2}$ [16], $\mathrm{LiCuO}_{2}[8,16]$ y CuO [8] (ver ecuaciones $(2-1)$ a $\left.(2-4)\right)^{\text {e }}$, así como cambiar los poliedros de coordinación Li-O (de tetraédricos a octaédricos, Figura 2-3) [16]. Por otro lado, la formación de $\mathrm{CuO}$, de acuerdo a (2-2) posiblemente es acompañada por la formación de $\mathrm{O}_{2}$ debido a la oxidación de los oxígenos presentes en el material durante este proceso [8]. Cuando el proceso inverso (descarga, litiación) toma lugar, no es posible restaurar todo el material a su estado inicial litiado debido a la liberación de $\mathrm{O}_{2}$ y la formación de las fases compactadas, dificultando la reinserción del ion litio y con ello, una disminución severa en la capacidad durante el primer ciclo de carga-descarga $[8,16]$ y que se repite en los ciclos posteriores [8].

En resumen, $\mathrm{Li}_{2} \mathrm{CuO}_{2}$ muestra una alta capacidad $\left(>200 \mathrm{~mA} \mathrm{~h} \mathrm{~g}{ }^{-1}\right)$ únicamente en la primera carga. Sólo una tercera parte de ésta es retenida durante la primera descarga, debido a la formación de un material multifase acompañado de liberación de $\mathrm{O}_{2}$ y que no es posible revertir completamente durante la descarga.

\subsection{Sistemas derivados de $\mathrm{Li}_{2} \mathrm{CuO}_{2}$}

Con el objetivo de estabilizar al $\mathrm{Li}_{2} \mathrm{CuO}_{2}$ durante el proceso redox para mejorar su desempeño como material catódico, se han realizado sustituciones de átomos de $\mathrm{Cu}$ en la estructura del cuprato por otros metales de transición obteniendo sistemas derivados. Setiawati y colaboradores sintetizaron materiales de fórmula general $\mathrm{Li}_{2} \mathrm{Cu}_{1-x} \mathrm{M}_{x} \mathrm{O}_{2}(\mathrm{M}=\mathrm{Co}, \mathrm{Ni}, \mathrm{Fe}$, Mn y Ti), donde $\mathrm{Li}_{2} \mathrm{Cu}_{0.7} \mathrm{Ni}_{0.3} \mathrm{O}_{2}$ y Li ${ }_{2} \mathrm{Cu}_{0.9} \mathrm{Co}_{0.1} \mathrm{O}_{2}$ mostraron ser los de mejor desempeño; el primero, mostró la mayor capacidad de descarga, mientras que el segundo la mayor capacidad de carga [20].

Por otro lado, Ruther y colaboradores sintetizaron el sistema derivado $\mathrm{Li}_{2} \mathrm{Cu}_{0.5} \mathrm{Ni}_{0.5} \mathrm{O}_{2}$, que mostró una mayor estabilidad que $\mathrm{Li}_{2} \mathrm{CuO}_{2}$ ante ciclos repetidos de carga-descarga, sin

\footnotetext{
${ }^{e} \mathrm{Li}_{1.5} \mathrm{CuO}_{2}$ y LiCuO${ }_{2}$ son más compactas que $\mathrm{Li}_{2} \mathrm{CuO}_{2}$ debido a la presencia de rugosidades (inclinación de
} los planos cuadrados $\mathrm{CuO}_{4}$ ). 


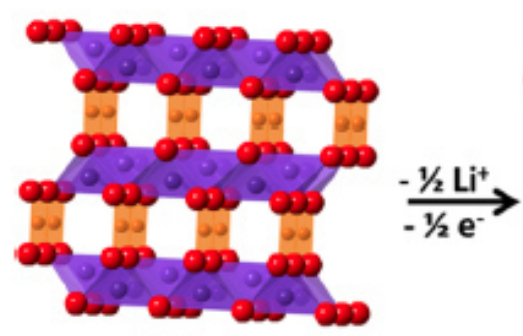

$\mathrm{Li}_{2} \mathrm{CuO}_{2}$ Immm orthorhombic $3.7 \mathrm{~g} / \mathrm{cm}^{3}$

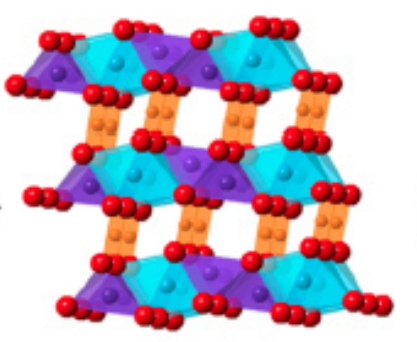

$\mathrm{Li}_{1.5} \mathrm{CuO}_{2}$ C2/m monoclinic

$4.0 \mathrm{~g} / \mathrm{cm}^{3}$

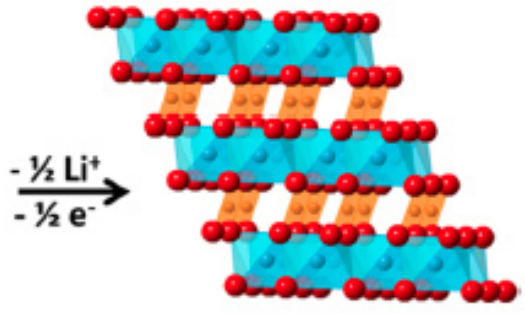

$\mathrm{LiCuO}_{2}$ $\mathrm{C2} / \mathrm{m}$ monoclinic $4.5 \mathrm{~g} / \mathrm{cm}^{3}$

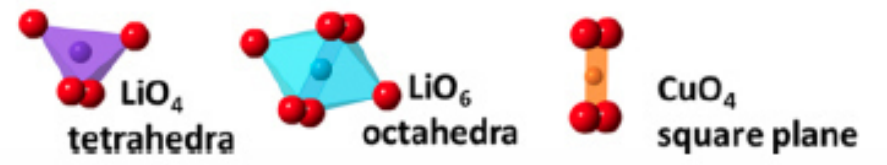

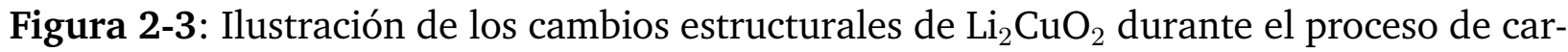
ga (arriba). Abajo se muestran los poliedros de coordinación Li-O formados durante este proceso. Las esferas rojas representan a los átomos de oxígeno, anaranjadas a cobre, azules y moradas a litio. Reimpreso con permiso de Chemistry of Materials, Ruther, R. E.; Zhou, H.; Dhital, C.; Saravanan, K.; Kercher, A. K.; Chen, G.; Huq, A.; Delnick, F. M.; Nanda, J., Synthesis, Structure, and Electrochemical Performance of High Capacity $\mathrm{Li}_{2} \mathrm{Cu}_{0.5} \mathrm{Ni}_{0.5} \mathrm{O}_{2}$ Cathodes, 2015, 27, 6746-6754. Copyright 2015 American Chemical Society.

embargo también presentó la compactación de la estructura laminar al deslitiarse, así como la participación redox del oxígeno a un voltaje mayor a 3.9V [16,21].

También se han sintetizado los sistemas $\mathrm{Li}_{2} \mathrm{Cu}_{0.5} \mathrm{Ni}_{0.4} \mathrm{Al}_{0.1} \mathrm{O}_{2}$ y Li $\mathrm{Cu}_{0.5} \mathrm{Ni}_{0.4} \mathrm{Ga}_{0.1} \mathrm{O}_{2}$, los cuales no mostraron mayor retención de la capacidad que $\mathrm{Li}_{2} \mathrm{CuO}_{2}$. Tal y como se aprecia en la (Tabla 2-2), a pesar de estos intentos no se ha conseguido aumentar el desempeño de $\mathrm{Li}_{2} \mathrm{CuO}_{2}$, lo que significa que es necesario obtener mayor información del proceso redox inherente a $\mathrm{Li}_{2} \mathrm{CuO}_{2}$, así como en materiales derivados. 


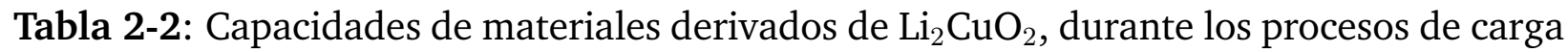
y descarga

\begin{tabular}{|c|c|c|c|c|}
\hline \multirow[t]{3}{*}{ Sistema } & \multicolumn{4}{|c|}{ Capacidad ( $\mathrm{mA} \mathrm{h} \mathrm{g}^{-1}$ ) } \\
\hline & \multicolumn{2}{|c|}{ Primer ciclo } & \multicolumn{2}{|c|}{$n$ ciclos } \\
\hline & Carga & Descarga & Carga & Descarga \\
\hline $\mathbf{L i}_{2} \mathbf{C u}_{0.7} \mathbf{N i}_{0.3} \mathbf{O}_{2}$ [20] & 250 & $\sim 150$ & - & - \\
\hline $\mathbf{L i}_{2} \mathbf{C u}_{0.9} \mathbf{C o}_{0.1} \mathbf{O}_{2}$ [20] & 270 & $\sim 140$ & - & - \\
\hline $\mathbf{L i}_{2} \mathbf{C u}_{0.5} \mathbf{N i}_{0.5} \mathbf{O}_{2}$ & 370 & 300 & $\sim 120^{\mathrm{a}}$ & $\sim 110^{\mathrm{a}}$ \\
\hline $\mathbf{L i}_{2} \mathbf{C u}_{0.5} \mathbf{N i}_{0.4} \mathbf{A l}_{0.1} \mathbf{O}_{2}$ & 188 & 161 & $\sim 100^{\mathrm{b}}$ & $100^{\mathrm{b}}$ \\
\hline $\mathbf{L i}_{2} \mathbf{C u}_{0.5} \mathbf{N i}_{0.4} \mathbf{G a}_{0.1} \mathbf{O}_{2}$ [22] & 234 & 204 & $\sim 120^{\mathrm{b}}$ & $114^{\mathrm{b}}$ \\
\hline
\end{tabular}

\subsection{Participación redox del oxígeno en cátodos basados en óxidos}

La importancia de la participación de los átomos de oxígeno en el proceso redox que sucede en óxidos de metales de transición utilizados como materiales catódicos en BIL, ha sido señalada desde hace años [5] y todavía es un campo activo de investigación [23]. La liberación de oxígeno es un factor que influye en la seguridad y desempeño de los materiales catódicos basados en óxidos metálicos, puede suceder cuando el óxido metálico es severamente deslitiado a partir de una sobrecarga o por sobrecalentamiento [24].

En el contexto de los óxidos laminares, la participación redox del oxígeno puede aumentar o disminuir el rendimiento del material, dependiendo de la naturaleza de éste. Por ejemplo, en los denominados materiales abundantes en litio, la oxidación del oxígeno puede aumentar la capacidad del material mas allá de la estimada, considerando únicamente la participación redox de los átomos de metal de transición [25-27], gracias a cierta reversibilidad de las especies oxigenadas en el proceso óxido/reducción de cada ciclo de carga/descarga, respectivamente [25, 28, 29]. Por otro lado, en los óxidos de metales de transición, la oxidación de los oxígenos ha sido relacionada con la formación de $\mathrm{O}_{2}[8,30]$, la cual ha sido atribuída a la oxidación no reversible de la submalla de aniones oxígeno a través de la formación de aniones peróxido [24]:

$$
\begin{gathered}
2 \mathrm{O}^{2-} \longrightarrow \mathrm{O}_{2}^{2-}+2 e^{-} \\
\mathrm{O}_{2}^{2-} \longrightarrow \mathrm{O}_{2}+2 e^{-}
\end{gathered}
$$

En el proceso de descarga, como se mencionó antes, $\operatorname{los} \mathrm{Li}^{+}$y $e^{-}$son insertados al cátodo. En un estudio teórico de cátodos basados en óxidos laminares tipo $\mathrm{LiMO}_{2}$ (donde $\mathrm{M}=$ 
Ti, V, Mn, Co, Ni, Cu, Zn o Al), se concluyó que la carga electrónica transferida en el proceso de litiación, es mayor, en todos los casos, hacia los átomos de oxígeno que a los metales de transición, y la tendencia se incrementa de $\mathrm{Ti}$ a $\mathrm{Zn}$ y Al, por lo que $\mathrm{LiTiO}_{2}$ presenta una menor disposición de oxígeno durante la carga [5]. Recientemente, Johannes y colaboradores [24] propusieron que mediante el análisis de la contribución de los estados de oxígeno a la densidad de estados (density of states, DOS), es posible conocer la tendencia a la oxidación de los aniones de oxígeno presentes en los materiales catódicos.

Desde el punto de vista experimental, la participación redox de oxígeno y por consiguiente, la liberación de $\mathrm{O}_{2}$ en $\mathrm{Li}_{2} \mathrm{CuO}_{2}$ [8] y $\mathrm{Li}_{2} \mathrm{Cu}_{0.5} \mathrm{Ni}_{0.5} \mathrm{O}_{2}$ [16], ha sido propuesta para explicar los cambios químicos y estructurales observados en los estudios espectroscópicos Raman y de rayos $\mathrm{X}$. Por otro lado, desde el marco de los estudios de estructura electrónica este fenómeno redox ha sido estudiado a través de:

1. la determinación de la carga electrónica sobre el oxígeno [5],

2. el mapeo de diferencias de densidad electrónica y

3. el análisis de la DOS cerca del nivel de Fermi $[8,24]$.

En el proceso de carga, la participación redox de oxígeno en $\mathrm{Li}_{2} \mathrm{CuO}_{2}$, ha sido relacionada con: 1) el cambio de la densidad electrónica después de la sustracción de carga y 2) la contribución de oxígeno en los estados cercanos al nivel de Fermi. Se ha mostrado que al retirar un electrón de $\mathrm{Li}_{2} \mathrm{CuO}_{2}$ y restar las densidades electrónicas, los sitios afectados corresponden al plano cuadrado de $\mathrm{CuO}_{4}$ (Figura 2-4) [8,24].

Por otro lado, el análisis de la densidad de estados proyectada (projected density of states, DOS-p) de $\mathrm{Li}_{2} \mathrm{CuO}_{2} \mathrm{y} \mathrm{LiCuO}_{2}$, sugiere que el estado desocupado por la deslitiación corresponde en mayor medida a oxígeno (Figura 2-5) [8,24], pero no es sencillo hacer una

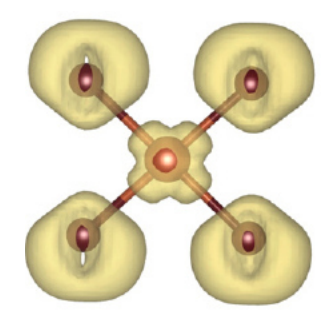

Figura 2-4: Diferencia de densidad electrónica después de la sustracción de un electrón en $\mathrm{Li}_{2} \mathrm{CuO}_{2}$. El átomo del centro corresponde a cobre y los demás son átomos de oxígeno. Reimpreso de Solid State Ionics, 286, Johannes, M. D.; SwiderLyons, K.; Love, C. T., Oxygen character in the density of states as an indicator of the stability of Li-ion battery cathode materials, 83-89, Copyright (2016), con permiso de Elsevier. 
comparación relativa directa.

La contribución más importante del presente trabajo fue utilizar la blandura local, $s(\mathbf{r})$ como alternativa a la $D O S$ - $p$, para el análisis del proceso de oxidación en $\mathrm{Li}_{2} \mathrm{CuO}_{2}$ y sistemas derivados $\mathrm{Li}_{2} \mathrm{Cu}_{1-x} \mathrm{M}_{x} \mathrm{O}_{2}$ y como se mencionará más adelante, fue posible realizar comparaciones directas entre diferentes sistemas, de forma más práctica que la DOS- $p$, todo esto en el marco de la teoría de los funcionales de la densidad (density functional theory, DFT).

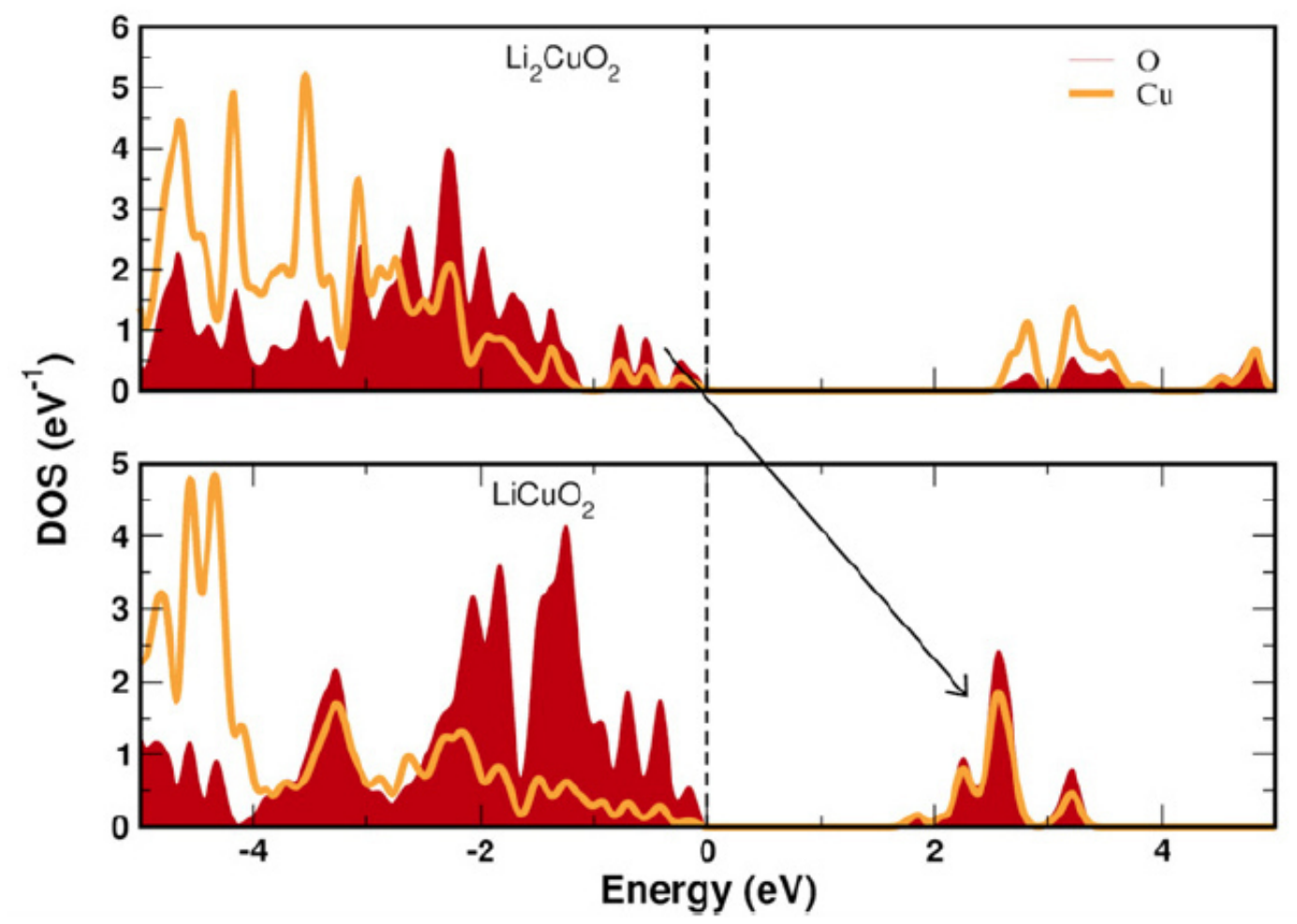

Figura 2-5: $D O S-p$ para $\mathrm{Cu}$ y $\mathrm{O}$ en $\mathrm{Li}_{2} \mathrm{CuO}_{2}$. Arriba se muestra el sistema antes de la carga (completamente litiado) y abajo en un estado parcialmente deslitiado. La flecha indica el estado que fue desocupado por la sustracción de un electrón en una primera etapa de deslitiación. Reimpreso de Solid State Ionics, 286, Johannes, M. D.; Swider-Lyons, K.; Love, C. T., Oxygen character in the density of states as an indicator of the stability of Li-ion battery cathode materials, 83-89, Copyright (2016), con permiso de Elsevier. 


\section{Capítulo}

\section{Marco Teórico}

El presente trabajo se desarrolló en el marco de la teoría de los funcionales de la densidad bajo condiciones periódicas, la cual se fundamenta en la ecuación de Schrödinger.

\subsection{Ecuación de Schrödinger}

La ecuación mecano-cuántica de Schrödinger [31] permite describir en su totalidad a cualquier sistema compuesto de núcleo(s) y electron(es) (tal como átomos, moléculas y cristales) [32]. Si la dependencia respecto al tiempo es omitida, la expresión es [32,33]

$$
\hat{H} \Psi=E \Psi
$$

en la cual, $\Psi=\Psi\left(\mathbf{x}_{1}, \mathbf{x}_{2}, \mathbf{x}_{3}, \ldots, \mathbf{x}_{N}, \mathbf{R}_{1}, \mathbf{R}_{2}, \mathbf{R}_{3}, \ldots, \mathbf{R}_{M}\right)$ es la función de onda del sistema bajo estudio y tiene dependencia de las coordenadas electrónicas $\left\{\mathbf{x}_{i}\right\}$ (tanto espaciales $\left\{\mathbf{r}_{i}\right\}$ como de espín $\left\{\mathbf{s}_{i}\right\}$ ) y nucleares espaciales $\left\{\mathbf{R}_{A}\right\}$ [34]. $E$ representa la energía del sistema, el valor propio (eigenvalue) que se obtiene cuando es aplicado el operador Hamiltoniano $\hat{H}$ sobre la función de onda $\Psi$ [32]. $\hat{H}$ es la suma de los operadores de energía cinética $\hat{T}$ y potencial $\hat{V}$

$$
\hat{H}=\hat{T}+\hat{V}
$$

el cual, para un sistema de $M$ núcleos, $N$ electrones y sin correcciones relativistas puede escribirse como [32]

$$
\hat{H}=-\overbrace{\sum_{i=1}^{N} \frac{\hbar^{2}}{2 m} \nabla_{i}^{2}-\sum_{A=1}^{M} \frac{\hbar^{2}}{2 M_{A}} \nabla_{A}^{2}}^{-\frac{1}{4 \pi \epsilon_{0}}\left(\sum_{i=1}^{N} \sum_{A=1}^{M} \frac{Z_{A} e^{2}}{r_{i A}}-\sum_{i=1}^{N} \sum_{j>i}^{N} \frac{e^{2}}{r_{i j}}-\sum_{A=1}^{M} \sum_{B>A}^{M} \frac{Z_{A} Z_{B} e^{2}}{R_{A B}}\right)}
$$


A partir de esta expresión pueden identificarse cinco términos. Los primeros dos surgen del hecho que tanto los núcleos como los electrones están en movimiento, es decir, describen las energías cinéticas de los $N$ electrones con masas $m$ y los $M$ núcleos con masas $M_{A}$, dónde $\nabla^{2}$ es el operador diferencial [32]

$$
\nabla^{2}=\nabla \cdot \nabla=\frac{\partial^{2}}{\partial x^{2}}+\frac{\partial^{2}}{\partial y^{2}}+\frac{\partial^{2}}{\partial z^{2}} \quad .
$$

Los últimos tres términos de la ecuación 3-3 describen la energía potencial debida a

1. La atracción de Coulomb entre los electrones (de carga $-e$ ) y los núcleos (de carga $Z_{A} e$ ) a una distancia $r_{i A}$ [32], donde el término:

$$
-\sum_{A=1}^{M} \frac{Z_{A} e^{2}}{r_{i A}}=v\left(\mathbf{r}_{i}\right)
$$

es conocido como el potencial externo $v\left(\mathbf{r}_{i}\right)$ experimentado por el $i$-ésimo electrón.

\section{Repulsión de Coulomb entre los electrones}

3. Repulsión de Coulomb entre los núcleos

Además de considerar la independencia del tiempo en la expresión explícita de $\hat{H}$ de la ecuación 3-3, puede introducirse la aproximación de Born-Oppenheimer, mediante la cual se obtiene un operador $\hat{H}_{\text {elec }}$ cuya función propia (eigenfunction), es una función de onda electrónica, que depende explícitamente de las coordenadas electrónicas y parámetricamente de las coordenadas nucleares. Dicha aproximación se justifica al considerar que las velocidades de los núcleos, debido a su mayor masa que la de los electrones, son mucho menores a la de los electrones, lo que permite calcular la contribución electrónica a las energías cinética y potencial a posiciones fijas en los núcleos. Como consecuencia, el segundo término de la ecuación 3-3 se hace cero; mientras que el quinto puede ser considerado una constante [32], de esta manera la ecuación 3-3 se reduce a la siguiente expresión en unidades atómicas [34] $\left(\hbar=m=e=4 \pi \epsilon_{0}=1[35,36]\right)$

$$
\hat{H}_{\text {elec }}=-\sum_{i=1}^{N} \frac{1}{2} \nabla_{i}^{2}-\sum_{i=1}^{N} \sum_{A=1}^{M} \frac{Z_{A}}{r_{i A}}+\sum_{i=1}^{N} \sum_{j>i}^{N} \frac{1}{r_{i j}}
$$

la cual queda representada como [32-34]

$$
\hat{H}_{\text {elec }}=\hat{T}_{\text {elec }}+\hat{V}_{n e}+\hat{V}_{e e} .
$$

La solución a la ecuación de Schrödinger 3-7 es la función de onda electrónica $\Psi_{\text {elec }}$ cuyo valor propio es la energía electrónica $E_{\text {elec }}[34]$ 


$$
\hat{H}_{\text {elec }} \Psi_{\text {elec }}=E_{\text {elec }} \Psi_{\text {elec }}
$$

a la cual es necesario adicionarle la energía de repulsión de Coulomb entre los núcleos para obtener la energía total del sistema bajo la aproximación de Born-Oppenheimer [33,34]

$$
E_{\text {total }}=E_{\text {elec }}+E_{\text {nuc }}
$$

donde $E_{n u c}$ está dado por [34]:

$$
\hat{V}_{n n}=\sum_{A=1}^{M} \sum_{B>A}^{M} \frac{Z_{A} Z_{B}}{R_{A B}} .
$$

\subsubsection{El principio variacional}

Resolver la ecuación de Schrödinger 3-7, requiere en primer lugar establecer específicamente el operador $\hat{H}_{\text {elec }}$ del sistema bajo estudio. De acuerdo a la ecuación 3-6, puede notarse que tanto el número de electrones $N$ del sistema como el potencial externo descrito en el operador $\hat{V}_{n e}$, son los que marcan la diferencia de un sistema a otro. Las expresiones explícitas de los operadores $\hat{T}_{\text {elec }}$ y $\hat{V}_{e e}$, son conocidas para cualquier sistema bajo estudio [34].

Una vez conocido el operador explícito $\hat{H}_{\text {elec }}$ del sistema, el siguiente paso es conocer el conjunto de funciones propias $\left\{\Psi_{i}\right\}$ de este $\hat{H}_{\text {elec }}$, de las cuales, nos interesa casi siempre aquella que describe al estado basal del sistema, es decir, $\Psi_{0}$, la cual en la mayoría de los casos, no se conoce de forma exacta. La mejor aproximación que se puede obtener a ésta, a través del principio variacional [34].

De acuerdo a la Mecánica Cuántica, el valor esperado de un observable en particular, queda expresado a través del operador apropiado $\hat{O}$ aplicado a una función de prueba, $\Psi_{\text {trial }}$, la cual es considerada en general compleja [33,34]

$$
\langle\hat{\mathrm{O}}\rangle=\int \cdots \int \Psi_{\text {trial }}^{*} \hat{\mathrm{O}} \Psi_{\text {trial }} \mathrm{d} \mathbf{x}_{1} \mathrm{~d} \mathbf{x}_{2} \ldots \mathrm{d} \mathbf{x}_{N} \equiv\left\langle\Psi_{\text {trial }}|\hat{\mathrm{O}}| \Psi_{\text {trial }}\right\rangle
$$

En la ecuación 3-11, se ha introducido la notación braket de Dirac. El asterisco en $\Psi_{\text {trial }}^{*}$ hace referencia al complejo conjugado de $\Psi_{\text {trial }}$, la cual se considera que está normalizada, por lo tanto [34]

$$
\int \cdots \int\left|\Psi_{\text {trial }}\left(\mathbf{x}_{1}, \mathbf{x}_{2}, \ldots, \mathbf{x}_{N}\right)\right|^{2} \mathrm{~d} \mathbf{x}_{1} \mathrm{~d} \mathbf{x}_{2} \ldots \mathrm{d} \mathbf{x}_{N}=1 .
$$

El principio variacional establece que la energía calculada mediante la ecuación 3-11, como el valor esperado del operador $\hat{H}_{\text {elec }}$ (denotado de ahora en adelante simplemente como 
$\hat{H}$ ) aplicado a cualquier $\Psi_{\text {trial }}$ propuesta, será una cota superior a la energía exacta del estado basal del sistema [33,34]

$$
\left\langle\Psi_{\text {trial }}|\hat{\mathrm{H}}| \Psi_{\text {trial }}\right\rangle=\mathrm{E}_{\text {trial }} \geq \mathrm{E}_{0}=\left\langle\Psi_{0}|\hat{\mathrm{H}}| \Psi_{0}\right\rangle
$$

donde la igualdad se mantiene únicamente en el caso de que $\Psi_{\text {trial }}$ sea idéntica a $\Psi_{0}$. Dada la ecuación 3-13, es necesario minimizar el funcional $E[\Psi]$ para determinar la energía del estado basal del sistema [34]

$$
\delta E[\Psi]=0
$$

sujeto a la condición 3-12, para lo cual se puede utilizar el método de los multiplicadores indeterminados de Lagrange y la ecuación 3-14 queda expresada como [35]

$$
\delta[\langle\Psi|\hat{\mathrm{H}}| \Psi\rangle-E(\langle\Psi \mid \Psi\rangle-1)]=0 .
$$

\subsubsection{Interacciones electrón-electrón}

El operador $\hat{H}_{\text {elec }}$ de la expresión 3-7, describe interacciones núcleo-electrón y electrónelectrón. El problema más difícil en cualquier cálculo de estructura electrónica radica en considerar los efectos de la interacción electrón-electrón [37].

La función de onda de un sistema de varios electrones debe ser antisimétrica [37], esto es, si dos electrones intercambian posiciones, la función de onda debe cambiar de signo, además, debe considerarse que cada electrón es afectado por el movimiento de los otros electrones en el sistema; esto es, el movimiento es correlacionado [38].

Un método de estructura electrónica. que cumple con el requisito de antisimetría en la función de onda es la aproximación Hartree-Fock (HF), la cual aproxima la función de onda de la ecuación de Schrödinger como un determinante de Slater [34]:

$$
\Psi_{0} \approx \Phi_{S D}=\frac{1}{\sqrt{N !}}\left|\begin{array}{cccc}
\chi_{1}\left(\mathbf{x}_{1}\right) & \chi_{2}\left(\mathbf{x}_{1}\right) & \ldots & \chi_{N}\left(\mathbf{x}_{1}\right) \\
\chi_{1}\left(\mathbf{x}_{2}\right) & \chi_{2}\left(\mathbf{x}_{2}\right) & \ldots & \chi_{N}\left(\mathbf{x}_{2}\right) \\
\vdots & \vdots & \vdots & \vdots \\
\chi_{1}\left(\mathbf{x}_{N}\right) & \chi_{2}\left(\mathbf{x}_{N}\right) & \ldots & \chi_{N}\left(\mathbf{x}_{N}\right)
\end{array}\right|
$$

donde cada $\chi_{i}\left(\mathbf{x}_{i}\right)$ representa un espín-orbital compuesto por un orbital espacial $\psi_{i}(\mathbf{r})$ y una función de espín, $\alpha(\mathrm{s})$ o $\beta$ (s)

$$
\chi_{i}(\mathbf{x})=\psi_{i}(\mathbf{r}) \sigma(\mathbf{s}), \quad \sigma=\alpha, \beta .
$$

Cabe mencionar que las funciones de espín son ortonormales, es decir, $\langle\alpha \mid \alpha\rangle=\langle\beta \mid \beta\rangle=1$ mientras que $\langle\alpha \mid \beta\rangle=\langle\beta \mid \alpha\rangle=0$. Además, generalmente también los espín-orbitales son 
seleccionados para que sean ortonormales:

$$
\int \chi_{i}^{*}(\mathbf{x}) \chi_{j}(\mathbf{x}) d \mathbf{x}=\left\langle\chi_{i} \mid \chi_{j}\right\rangle=\delta_{i j} \quad \delta_{i j}=\left\{\begin{array}{ll}
1 & \text { si } i=j \\
0 & \text { si } i \neq j
\end{array} .\right.
$$

Al utilizar el principio variacional, la energía es minimizada respecto a la selección de los espín-orbitales, bajo la restricción de la ecuación 3-18 siendo $\epsilon_{i}$ los multiplicadores de Lagrange en las ecuaciones resultantes, es decir, las ecuaciones de HF [34,39]:

$$
\hat{f} \chi_{i}=\epsilon_{i} \chi_{i}, \quad i=1,2, \ldots, N
$$

donde los multiplicadores indeterminados de Lagrange, $\epsilon_{i}$, son los valores propios (energías orbitales) del operador de HF monoelectrónico, $f$, el cual es definido como

$$
\hat{f}=-\frac{1}{2} \nabla_{i}^{2}-\sum_{A}^{M} \frac{Z_{A}}{r_{i A}}+V_{H F}(i)
$$

en el cual el primer término representa el operador de energía cinética del $i$-ésimo electrón, el segundo término describe el operador de energía potencial entre los núcleos y el $i$ ésimo electrón. Por ultimo, $V_{H F}$, es el potencial de $H F$, el cual es el potencial promedio experimentado por el $i$-ésimo electrón debido a los $N-1$ electrones restantes y consta de dos operadores

$$
V_{H F}\left(\mathbf{x}_{1}\right)=\sum_{j}^{N}\left(\hat{\mathbf{J}}_{j}\left(\mathbf{x}_{1}\right)-\hat{\mathbf{K}}_{j}\left(\mathbf{x}_{1}\right)\right)
$$

en la cual $\hat{\mathbf{J}}_{j}$ es el operador de Coulomb expresado cuando opera sobre un espín-orbital

$$
\hat{\mathbf{J}}_{j}\left(\mathbf{x}_{1}\right) \chi_{i}\left(\mathbf{x}_{1}\right)=\int\left|\chi_{j}\left(\mathbf{x}_{2}\right)\right|^{2} \frac{1}{r_{12}} d \mathbf{x}_{2} \chi_{i}\left(\mathbf{x}_{1}\right)
$$

y representa la repulsión que un electrón en la posición $\mathrm{x}_{1}$ experimenta, debido a la distribución de carga de otro electrón en el espín-orbital $\chi_{j}$. Este operador se dice que es local, porque su aplicación sobre un espín-orbital $\chi_{i}\left(\mathbf{x}_{1}\right)$ depende únicamente del valor de $\chi_{i}$ en la posición $\mathrm{x}_{1}$.

El segundo operador en la ecuación 3-21 es el operador de intercambio $\hat{\mathbf{K}}$, el cual no tiene interpretación clásica y únicamente puede ser definido a través de su efecto cuando opera sobre un espín-orbital

$$
\hat{\mathbf{K}}_{j}\left(\mathbf{x}_{1}\right) \chi_{i}\left(\mathbf{x}_{1}\right)=\int \chi_{j}^{*}\left(\mathbf{x}_{2}\right) \frac{1}{r_{12}} \chi_{i}\left(\mathbf{x}_{2}\right) d \mathbf{x}_{2} \chi_{j}\left(\mathbf{x}_{1}\right)
$$


el cual lleva al intercambio de las variables en los dos espín-orbitales. A diferencia del operador de Coulomb, el operador de Intercambio es un operador no local, ya que su operación sobre $\chi_{i}\left(\mathbf{x}_{1}\right)$ depende del valor de $\chi_{i}$ sobre todos los puntos en el espacio [34].

La diferencia entre la energía exacta no relativista y la energía calculada en la aproximación Hartree-Fock es llamada la Energía de Correlación, la cual es difícil de calcular [37], por lo que existen diferentes aproximaciones a este término.

\subsection{Teoría de los funcionales de la densidad}

La DFT es un método de estructura electrónica que se basa en expresar la energía como un funcional de la densidad electrónica. Esta teoría descansa en los teoremas de Hohenberg y Kohn (1964) [40]. Gracias al trabajo de Kohn y Sham [41], la DFT hoy en día es uno de los métodos de estructura electrónica más populares.

\subsubsection{Los teoremas de Hohenberg y Kohn}

El primer teorema de Hohenberg y Kohn (HK) puede titularse como prueba de existencia [34] y establece lo siguiente [40]:

El potencial externo, $v(\mathbf{r})$, es (hasta una constante) un funcional único de la densidad electrónica $\rho(\mathbf{r})$ y como $v(\mathbf{r})$ establece a $\hat{H}$, entonces, la energía del estado basal del sistema $E_{0}$, es un funcional único de la densidad electrónica del estado basal, $\rho_{0}(\mathbf{r})$.

Este teorema quiere decir que si conocemos la densidad electrónica del estado basal $\rho_{0}(\mathbf{r})$, entonces conocemos el el potencia externo $v(\mathbf{r})$ asociado a ella y de esta forma se puede establecer el operador hamiltoniano $\hat{H}$ que nos permitirá conocer la función de onda del estado basal y con ello la energía así como todas las otras propiedades del sistema [34]:

$$
\rho_{0}(\mathbf{r}) \Rightarrow\left\{N, Z_{A}, \mathbf{R}_{A}\right\} \Rightarrow \hat{H} \Rightarrow \Psi_{0} \Rightarrow E_{0} \text { (y todas las otras propiedades) } .
$$

Esto permite escribir a la energía $E_{v}{ }^{a}$, como un funcional de la densidad electrónica, cuyos componentes son la energía cinética $T[\rho(\mathbf{r})]$, energía potencial electrón-electrón $V_{e e}[\rho(\mathbf{r})]$ y la energía potencial núcleo-electrón $V_{n e}[\rho(\mathbf{r})][33]$ :

$$
\begin{aligned}
E_{v}[\rho(\mathbf{r})] & =T[\rho(\mathbf{r})]+V_{e e}[\rho(\mathbf{r})]+V_{n e}[\rho(\mathbf{r})] \\
& =F_{H K}[\rho(\mathbf{r})]+\int \rho(\mathbf{r}) v(\mathbf{r}) d \mathbf{r}
\end{aligned}
$$

${ }^{a}$ el subíndice es para hacer explícita la dependencia en el potencial externo $v$. 
donde $F_{H K}[\rho(\mathbf{r})]$ es el funcional de Hohenberg y Kohn [33]:

$$
F_{H K}[\rho(\mathbf{r})]=T[\rho(\mathbf{r})]+V_{e e}[\rho(\mathbf{r})]
$$

el cual es universalmente válido para cualquier sistema, ya que no depende del potencial externo [34]. La demostración de la correpondencia uno a uno entre la densidad electrónica $\rho(\mathbf{r})$ y el potencia externo $v(\mathbf{r})$, consiste en reductio ad absurdum (reducción al absurdo) y puede encontrarse en las referencias [33-35,40].

Por otro lado, el segundo teorema de HK establece el principio variacional a $E_{v}[\rho(\mathbf{r})]$ [33]: Para una densidad de prueba $\tilde{\rho}(\mathbf{r})$, tal que

$$
\tilde{\rho}(\mathbf{r}) \geq 0
$$

$y$

$$
\int \tilde{\rho}(\mathbf{r}) d \mathbf{r}=N
$$

la energía $E_{v}[\tilde{\rho}(\mathbf{r})]$, será una cota superior a la energía verdadera del estado basal del sistema:

$$
E_{v}\left[\rho_{0}(\mathbf{r})\right] \leq E_{v}[\tilde{\rho}(\mathbf{r})] .
$$

Análogo a la sección 3.1.1, es necesario minimizar $E_{v}[\tilde{\rho}(\mathbf{r})]$ sujeto a la condición 3-28 lo cual nos lleva a la expresión

$$
\delta\left\{E_{v}[\tilde{\rho}(\mathbf{r})]-\mu\left(\int \tilde{\rho}(\mathbf{r}) d \mathbf{r}-N\right)\right\}=0
$$

donde $\mu$ es el multiplicador indeterminado de Lagrange y representa al potencial químico de los electrones [33].

\subsubsection{La ecuación de Kohn-Sham (KS)}

Un operador hamiltoniano para un sistema de partículas independientes bajo la influencia de un potencial externo es [33]:

$$
\hat{H}_{S}=\sum_{I}^{N}\left(-\frac{1}{2} \nabla_{i}^{2}\right)+\sum_{i}^{N} v_{S}\left(\mathbf{r}_{\mathbf{i}}\right) .
$$

Para el cual la función de onda exacta es un determinante de Slater [33, 34]:

$$
\Theta_{S}=\frac{1}{\sqrt{N !}}\left|\begin{array}{cccc}
\varphi_{1}\left(\mathbf{x}_{1}\right) & \varphi_{2}\left(\mathbf{x}_{1}\right) & \ldots & \varphi_{N}\left(\mathbf{x}_{1}\right) \\
\varphi_{1}\left(\mathbf{x}_{2}\right) & \varphi_{2}\left(\mathbf{x}_{2}\right) & \ldots & \varphi_{N}\left(\mathbf{x}_{2}\right) \\
\vdots & \vdots & \vdots & \vdots \\
\varphi_{1}\left(\mathbf{x}_{N}\right) & \varphi_{2}\left(\mathbf{x}_{N}\right) & \ldots & \varphi_{N}\left(\mathbf{x}_{N}\right)
\end{array}\right|
$$




$$
\left\langle\varphi_{i} \mid \varphi_{j}\right\rangle=\delta_{i j}
$$

donde $\varphi_{i}$ son espín orbitales ${ }^{\mathrm{b}}$ ortonormales (ecuación 3-33) y la energía cinética puede expresarse exactamente como [34]:

$$
T_{S}=-\frac{1}{2} \sum_{i}^{N}\left\langle\varphi_{i}\left|\nabla^{2}\right| \varphi_{i}\right\rangle
$$

En la sección anterior, a través de los teoremas de Hohenberg y Kohn (HK), la energía total del sistema se expresó en términos de la energía potencial núcleo-electrón y del funcional de HK, ambos como funcionales de la densidad electrónica (ecuación 3-26). En 1965, W. Kohn y L. J. Sham [41] propusieron que la densidad exacta del estado basal puede expresarse como:

$$
\rho_{0}(\mathbf{r})=\sum_{i}^{N} \sum_{s}\left|\varphi_{i}\right|^{2}
$$

la cual corresponde a la expresión de la densidad electrónica de un sistema de partícula independiente bajo un potencial externo local, cuyas representaciones de la función de onda y energía cinética exactas, corresponden a las ecuaciones (3-32 y 3-34), donde los orbitales $\varphi_{i}$ son conocidos como los orbitales de Kohn-Sham obtenidos al resolver las ecuaciones de KS con el potencial de intercambio y correlación (XC) exacto. Esta condición implica que, es posible expresar la densidad electrónica del estado basal de un sistema interactuante a partir de la expresión para la densidad electrónica de un sistema no interactuante $[34,35]$. Con esto en mente, KS introdujeron la siguiente separación del funcional $F_{H K}[\rho(\mathbf{r})][33,34]:$

$$
F_{H K}[\rho(\mathbf{r})]=T_{S}[\rho(\mathbf{r})]+J[\rho(\mathbf{r})]+E_{x c}[\rho(\mathbf{r})]
$$

donde $T_{S}[\rho(\mathbf{r})]$ representa la energía cinética del sistema de partículas independientes ${ }^{\mathrm{c}}$.

$J[\rho(\mathbf{r})]$ representa la repulsión electrón-electrón clásica y $E_{x c}[\rho(\mathbf{r})]$ es la energía de intercambio y correlación (XC) [33-35]:

$$
E_{x c}[\rho(\mathbf{r})] \equiv\left(T[\rho(\mathbf{r})]-T_{S}[\rho(\mathbf{r})]\right)+\left(V_{e e}[\rho(\mathbf{r})]-J[\rho(\mathbf{r})]\right) \quad .
$$

Todo esto lleva a expresar la ecuación 3-25 como [35]:

${ }^{\mathrm{b}}$ donde la variable $\mathbf{x}_{i}$ representa tanto al conjunto de coordenadas espaciales $\left\{\mathbf{r}_{i}\right\}$ como de espín $\left\{\mathbf{s}_{i}\right\}$

${ }^{\mathrm{c}}$ Cabe mencionar que la energía cinética $T_{S}[\rho(\mathbf{r})]$ no es igual a la energía cinética exacta ya que carece de la descripción del efecto que siente cada electrón por el movimiento de los demás, es decir, la correlación electrónica; aunque las funciones que se usan para la construcción de este término son las que producen la densidad exacta del estado basal con la expresión de partícula independiente. 


$$
\begin{aligned}
E_{K S}[\rho(\mathbf{r})] & =T_{S}[\rho(\mathbf{r})]+J[\rho(\mathbf{r})]+E_{x c}[\rho(\mathbf{r})]+E_{n e}[\rho(\mathbf{r})] \\
& =-\frac{1}{2} \sum_{i}^{N}\left\langle\varphi_{i}\left|\nabla^{2}\right| \varphi_{i}\right\rangle+\frac{1}{2} \iint \frac{\rho(\mathbf{r}) \rho\left(\mathbf{r}^{\prime}\right)}{\left|\mathbf{r}-\mathbf{r}^{\prime}\right|} d \mathbf{r} d \mathbf{r}^{\prime}+E_{x c}[\rho(\mathbf{r})]+\int v_{n e}(\mathbf{r}) \rho(\mathbf{r}) d \mathbf{r}
\end{aligned}
$$

de la cual, el término $E_{x c}[\rho(\mathbf{r})]$ es el único para el cual no hay una expresión conocida, por lo que existen diversas aproximaciones a este funcional (siguiente sección).

Una vez obtenida la expresión de la energía de KS, el siguiente paso es aplicar el principio variacional y minimizar la energía respecto a los orbitales de KS bajo la restricción de ortonormalidad de los mismos, esto es $[33,34]$ :

$$
\delta \Omega\left[\left\{\varphi_{i}\right\}\right]=\delta\left[E_{K S}[\rho(\mathbf{r})]-\sum_{i}^{N} \sum_{j}^{N} \epsilon_{i j}\left(\left\langle\varphi_{i} \mid \varphi_{j}\right\rangle-\delta_{i j}\right)\right]=0
$$

obteniendo al final las ecuaciones de Kohn-Sham, las cuales parecen ecuaciones de partícula independiente pero debido al término de XC que contiene los efectos de varios cuerpos, en realidad no lo son, siendo esta la gran ventaja de las ecuaciones de KS:

$$
\begin{aligned}
\hat{f}^{K S} \varphi_{i} & =\epsilon_{i} \varphi_{i} \\
{\left[-\frac{1}{2} \nabla^{2}+V_{e f e c}(\mathbf{r})\right] \varphi_{i} } & =\epsilon_{i} \varphi_{i}
\end{aligned}
$$

donde $V_{\text {efec }}(\mathbf{r})$ es conocido como el potencial de KS, el cual está constituido por tres potenciales [33]:

$$
\begin{aligned}
V_{\text {efec }}(\mathbf{r}) & =V_{\text {Hartree }}(\mathbf{r})+V_{x c}(\mathbf{r})+V_{\text {ext }}(\mathbf{r}) \\
& =\int \frac{\rho\left(\mathbf{r}^{\prime}\right)}{\left|\mathbf{r}-\mathbf{r}^{\prime}\right|} d \mathbf{r}^{\prime}+\frac{\delta E_{x c}[\rho(\mathbf{r})]}{\delta \rho(\mathbf{r})}+v_{n e}(\mathbf{r})
\end{aligned}
$$

$V_{\text {Hartree }}(\mathbf{r})$ es conocido como el potencial de Hartree y representa la repulsión clásica electrón-electrón; $V_{x c}(\mathbf{r})$ es el potencial de XC y $V_{e x t}(\mathbf{r})$ es el potencial externo que describe la interacción núcleo-electrón [35]. Dado que la expresión del término $E_{x c}[\rho(\mathbf{r})]$ no es conocida [34,35], el potencial $V_{x c}(\mathbf{r})$ es expresado únicamente como su derivada funcional respecto a la densidad electrónica. 


\subsection{Funcionales de intercambio-correlación}

Hasta la fecha no es conocida la forma exacta para la energía de XC $E_{x c}[\rho(\mathbf{r})]$ [34] (el tercer término del lado derecho de la ecuación 3-38), por lo que se cuenta con diferentes aproximaciones a este término. Generalmente la $E_{x c}[\rho(\mathbf{r})]$ es escrita como una suma de la energía de intercambio y la energía de correlación, cada una expresada en términos de una energía por partícula de la siguiente forma general [42]:

$$
E_{x c}[\rho(\mathbf{r})]=E_{x}[\rho(\mathbf{r})]+E_{c}[\rho(\mathbf{r})]=\int \rho(\mathbf{r}) \epsilon_{x}[\rho(\mathbf{r})] d \mathbf{r}+\int \rho(\mathbf{r}) \epsilon_{c}[\rho(\mathbf{r})] d \mathbf{r}
$$

donde $\epsilon_{x}[\rho(\mathbf{r})]$ y $\epsilon_{c}[\rho(\mathbf{r})]$ representan las energías por partícula de XC, respectivamente. La $E_{x}[\rho(\mathbf{r})]$, está dada como una suma de contribuciones de las densidades de espín $\alpha \mathbf{y} \beta$, debido a que el intercambio es un efecto presente sólo entre electrones del mismo espín; lo cual no es el caso en la $E_{c}[\rho(\mathbf{r})]$ que considera contribuciones del mismo espín así como de espín opuesto. La densidad total es la suma de las contribuciones $\alpha$ y $\beta$

$$
\rho(\mathbf{r})=\rho_{\alpha}(\mathbf{r})+\rho_{\beta}(\mathbf{r}) \quad .
$$

Sin embargo, los funcionales a menudo son expresados como función de la polarización de espín $\zeta^{\mathrm{d}} \mathrm{y}$ el radio del volumen efectivo que contiene a un electrón, $r_{s}$ [42]

$$
\begin{aligned}
& \zeta=\frac{\rho_{\alpha}(\mathbf{r})-\rho_{\beta}(\mathbf{r})}{\rho_{\alpha}(\mathbf{r})+\rho_{\beta}(\mathbf{r})} \\
& \frac{4}{3} \pi r_{s}^{3}=\rho(\mathbf{r})^{-1} .
\end{aligned}
$$

Las siguientes expresiones de la energía de XC contienen contribuciones de ambas densidades $\left(\rho_{\alpha}(\mathbf{r})\right.$ y $\left.\rho_{\beta}(\mathbf{r})\right)$.

Una forma de agrupar y clasificar a los funcionales de XC ha sido la escalera de Jacob propuesta por J. P. Perdew [36]. A continuación se describen brevemente algunos peldaños de esta escalera, relevantes para este trabajo.

\subsubsection{Aproximación de la densidad local}

La aproximación de la densidad local (local density approximation, $L D A$ ) es la formulación más básica para describir la $E_{x c}[\rho(\mathbf{r})]$. En ella se asume que la densidad es una función que varía lentamente en el espacio y se utiliza la forma proveniente de un gas de electrones uniforme [42]. La energía de XC es representada como [32]:

$$
E_{x c}^{L D A}[\rho(\mathbf{r})]=\int d \mathbf{r} \rho(\mathbf{r}) \epsilon_{x c}^{u n i f}[\rho(\mathbf{r})]
$$

${ }^{\mathrm{d}}$ La diferencia normalizada entre $\rho_{\alpha} \mathrm{y} \rho_{\beta}$ 
en la cual, $\epsilon_{x c}^{u n i f}[\rho(\mathbf{r})]$ es la energía de XC por partícula de un gas de electrones uniforme y es un funcional de la densidad electrónica $\rho(\mathbf{r})$.

La correspondiente versión LDA dependiente de espín es llamada aproximación de la densidad local de espín (local spin density approximation, $L S D A)[32,42]$,donde la $E_{x c}[\rho(\mathbf{r})]$ es un funcional de las densidades electrónicas de espín $\rho_{\alpha}(\mathbf{r})$ y $\rho_{\beta}(\mathbf{r})$ [36]. Para sistemas de capa cerrada ${ }^{\mathrm{e}}$, LSDA es igual a LDA [42].

\subsubsection{Aproximación del gradiente generalizado}

Una mejora a LSDA es considerar al gas electrónico como no uniforme, e incorporar esto en las energías de XC mediante su dependencia en la densidad electrónica $\rho(\mathbf{r})$ así como en su gradiente $\nabla \rho(\mathbf{r})$. Esto es conocido como la aproximación del gradiente generalizado (generalized gradient approximation, GGA) [36,42], cuya energía de XC puede ser expresada como $[36,44]$ :

$$
E_{x c}^{G G A}[\rho(\mathbf{r})]=\int d \mathbf{r} \rho(\mathbf{r}) \epsilon_{x c}^{G G A}[\rho(\mathbf{r}), \nabla \rho(\mathbf{r})]
$$

donde $\epsilon_{x c}^{G G A}[\rho(\mathbf{r}), \nabla \rho(\mathbf{r})]$ es la energía de XC con dependencia de la densidad electrónica y su gradiente. Una forma de incluir el $\nabla \rho(\mathbf{r})$ en la energía de XC es como una corrección a las energías de XC LDA [32]. Un ejemplo de esto es el funcional GGA de XC PBE [45, 46] cuya expresión de la energía de intecambio por partícula $\epsilon_{x}^{G G A}[\rho(\mathbf{r}), \nabla \rho(\mathbf{r})]$ es la siguiente $[44,45]$ :

$$
\epsilon_{x}^{P B E}[\rho(\mathbf{r}), \nabla \rho(\mathbf{r})]=\epsilon_{x}^{L D A}[\rho(\mathbf{r})] F^{P B E}(s),
$$

donde $F$ es conocido como el factor de exacerbamiento de PBE y está dado por [45]:

$$
\begin{aligned}
F^{P B E}(s) & =1+\kappa-\frac{\kappa}{\left(1+\frac{\mu}{\kappa} s^{2}\right)}, \\
\kappa & =0.804 \quad \mathrm{y} \\
\mu & =0.21951 .
\end{aligned}
$$

$F$ depende del gradiente adimensional de la densidad $s[45]$

$$
s=\frac{|\nabla \rho(\mathbf{r})|}{2 k_{F} \rho(\mathbf{r})}=\frac{|\nabla \rho(\mathbf{r})|}{2 \rho(\mathbf{r})^{\frac{4}{3}}} .
$$

Por otro lado, la energía de correlación por partícula puede ser representada como $[44,45]$ :

$$
\epsilon_{c}^{P B E}[\rho(\mathbf{r}), \nabla \rho(\mathbf{r})]=\epsilon_{c}^{L D A}\left[r_{s}, \zeta\right]+H\left[r_{s}, \zeta, t\right]
$$

\footnotetext{
${ }^{\mathrm{e}}$ sistemas en los cuales, cada uno de sus niveles son ocupados por dos electrones de espín opuesto [43].
} 
donde la función $H$ es una función que depende de constantes físicas, como la $r_{s}, \zeta$ y la $t[45]$ :

$$
\begin{gathered}
t=\frac{|\nabla \rho(\mathbf{r})|}{2 \phi(\zeta) k_{s} \rho(\mathbf{r})} \\
\phi(\zeta)=\frac{1}{2}\left[(1+\zeta)^{\frac{2}{3}}+(1-\zeta)^{\frac{2}{3}}\right] \\
k_{s}=\left(\frac{4 k_{F}}{\pi}\right)^{\frac{1}{2}} \\
k_{F}=\left[3 \pi^{2} \rho(\mathbf{r})\right]^{\frac{1}{3}}
\end{gathered}
$$

donde $\phi(\zeta)$ es el factor de escalamiento de espín, dado en función de la polarización de espín $\zeta$ (ecuación 3-44). $k_{s}$ es el vector de onda proyectado de Thomas-Fermi y $k_{F}$ representa al vector de onda local de Fermi [44].

\subsubsection{Funcionales con correcciones tipo Hubbard}

Los métodos basados en los funcionales convencionales LDA o GGA presentan problemas al describir sistemas que contiene metales de transición, tal como en el caso de óxidos de metales de transición, en los cuales la tendencia de estos funcionales es predecir estados basales metálicos, aunque experimentalmente se sabe que son de tipo aislante [47].

Lo anterior es asociado con una descripción inadecuada de la fuerte repulsión de Coulomb entre electrones $3 d$ de los iones metálicos [48], que sobre-deslocaliza los electrones de valencia y sobre-estabiliza estados basales metálicos. La sobre-deslocalización de los electrones puede deberse a que el funcional de XC falla en cancelar completamente la autointeracción electrónicaf contenida en el término de interacción electrón-electrón, por lo que al no ser cancelada completamente, la fracción restante induce una sobre-deslocalización de la función de onda [47].

Una opción para mejorar la descripción de la estructura electrónica de este tipo de sistemas, ha sido la adición de una corrección tipo Hubbard [49-54] a los estados electrónicos fuertemente correlacionados [55-59], tales como $d$ o $f$, mientras que el resto de los electrones de valencia son tratados a nivel del funcional estándar (LDA o GGA) dando origen a la aproximación $\mathrm{LDA}+\mathrm{U}$ (si la corrección es hecha a un funcional XC tipo LDA) o GGA+U(si la corrección es hecha a un funcional XC tipo GGA). La energía total es representada como [47]:

$$
E_{L D A+U}[\rho(\mathbf{r})]=E_{L D A}[\rho(\mathbf{r})]+E_{H u b}\left[\left\{\boldsymbol{n}_{m m^{\prime}}^{l \sigma}\right\}\right]-E_{d c}\left[\left\{\boldsymbol{n}^{l \sigma}\right\}\right]
$$

${ }^{\mathrm{f}}$ La auto-interacción electrónica puede ser interpretada como una repulsión de un electrón consigo mismo [47]. 
en la cual

$$
\boldsymbol{n}_{m m^{\prime}}^{l \sigma}=\sum_{k v} f_{k v}^{\sigma}\left\langle\psi_{k v}^{\sigma} \mid \phi_{m^{\prime}}^{l}\right\rangle\left\langle\phi_{m}^{l} \mid \psi_{k v}^{\sigma}\right\rangle
$$

La ecuación 3-57 representa la proyección de los orbitales de KS $\psi_{k v}^{\sigma}$ en un conjunto de bases localizadas $\phi_{m}^{l \mathrm{~h}}$ con sus respectivos coeficientes de ocupación $f_{k v}^{\sigma}$ i .

De la expresión 3-56 puede interpretarse que a la energía total obtenida utilizando un funcional XC tipo LDA $E_{L D A}[\rho(\mathbf{r})]$, se le adiciona la contribución de la interacción electrónelectrón de los estados $d$ o $f$ bajo el modelo de Hubbard $E_{H u b}\left[\left\{\boldsymbol{n}_{m m^{\prime}}^{l \sigma}\right\}\right]$, el cual es una función del conjunto de los números de ocupación (ecuación 3-57). Posteriormente se le resta la contribución de la interacción electrón-electrón modelada por $E_{H u b}$ que de alguna manera ya está considera dentro de $E_{L D A}$, todo esto a través del término ${ }^{\mathrm{k}} E_{d c}\left[\left\{\boldsymbol{n}^{l \sigma}\right\}\right]$, el cual es un funcional del promedio de los números de ocupación por sitiol. Cabe mencionar que no hay expresión única para $E_{d c}\left[\left\{\boldsymbol{n}^{l \sigma}\right\}\right]$, por lo que existen diferentes definiciones para este término. En el presente trabajo se ocupó la definición de $E_{d c}\left[\left\{\boldsymbol{n}^{l \sigma}\right\}\right]$ de acuerdo a Dudarev y colaboradores [60] y se utilizó el funcional PBE, por lo que la energía total queda expresada como:

$$
E_{P B E+U}[\rho(\mathbf{r})]=E_{P B E}[\rho(\mathbf{r})]+E_{U}\left[\left\{\boldsymbol{n}_{m m^{\prime}}^{l \sigma}\right\}\right]
$$

donde el término $E_{U}\left[\left\{\boldsymbol{n}_{m m^{\prime}}^{l \sigma}\right\}\right]$ queda expresado como [47]:

$$
E_{U}\left[\left\{\boldsymbol{n}_{m m^{\prime}}^{l \sigma}\right\}\right]=\sum_{l, \sigma} \frac{U^{l}}{2} \operatorname{Tr}\left[\left\{\boldsymbol{n}^{l \sigma}\left(1-n^{l \sigma}\right)\right\}\right]
$$

La ecuación 3-59 contiene tanto la corrección de Hubbard (el primer término del lado derecho de la ecuación) como el término de conteo doble (el segundo término del lado derecho de la ecuación). Cabe mencionar que la matriz $\boldsymbol{n}^{l \sigma}$ es una suma de matrices

$$
\boldsymbol{n}^{l \sigma}=\sum_{m} \boldsymbol{n}_{m}^{l \sigma}
$$

La forma de la ecuación 3-59 trae dos ventajas:

1. La forma del término de conteo doble queda expresada en términos de un sólo parámetro de interacción $U^{l}$ que, en la jerga se conoce como $U_{\text {efectivo }}$, etiquetado por átomo y puede ser visto como la resta de las interacciones efectivas de Coulomb e intercambio

\footnotetext{
getiquetados por punto $k$, banda $v$ y espín $\sigma$.

hetiquetada por un índice de sitio $l$ y uno de estado $m$.

${ }^{i}$ también etiquetados por punto $k$, banda $v$ y espín $\sigma$.

${ }^{\mathrm{j}}$ Estas son matrices etiquetadas por un índice de sitio $l$, espín $\sigma$ y estado $m$.

${ }^{k}$ Es conocido como término de conteo doble.

${ }^{1}$ Estas son matrices etiquetadas por un índice de sitio $l$ y uno espín $\sigma$.
} 
( $U$ y $J$ respectivamente) donde ésta última se considerada cero bajo el esquema de Duddarev [47].

2. La matriz de ocupaciones $\boldsymbol{n}^{l \sigma}$ queda invariante ante una transformación unitaria de la base seleccionada $\phi_{m}^{l}$, que define la proyección en las regiones atómicas y por consecuencia las ocupaciones atómicas. Esto genera que los cálculos no sean afectados por un cambio de la base de proyección.

\subsubsection{Aproximación del funcional de rango separado HSE06}

Describir la estructura electrónica de sistemas con electrones en estados $d$ o $f$ puede implicar una tarea no sencilla para los funcionales LDA y GGA convencionales de la DFT, esto debido a que la auto-interacción afecta más a este tipo de estados. Una alternativa a esta situación ha sido agregar una corrección (mediante el modelo de Hubbard) solamente a este tipo de estados electrónicos, tal y como se mencionó en el apartado anterior. Sin embargo, existe otra opción que consiste en utilizar funcionales híbridos, es decir, funcionales XC que incluyen una cierta cantidad de intercambio exacto [61]. Esta opción es más adecuada, en el sentido de que todos los estados electrónicos son evaluados bajo el mismo nivel de teoría [24]. Sin embargo, el precio a pagar es que son computacionalmente demandantes en sólidos y sistemas moleculares grandes [61].

Con esta situación en contexto, Heyd y colaboradores [61], propusieron en 2003 un funcional que incluyera el componente de intercambio exacto pero sólo de corto alcance, dando origen al funcional de rango separado hoy conocido como HSE06. La idea básica fue:

Paso 1 Tomar al funcional híbrido PBE0 [62]

$$
E_{x c}^{P B E 0}=a E_{x}^{H F}+(1-a) E_{x}^{P B E}+E_{c}^{P B E}
$$

donde $a=\frac{1}{4}$ es el coeficiente de mezclado y $E_{x}^{P B E 0}$ es

$$
E_{x}^{P B E 0}=a E_{x}^{H F}+(1-a) E_{x}^{P B E}
$$

Paso 2 Representar al operador de Coulomb en componentes de corto (SR) y largo alcance $(L R)$

$$
\frac{1}{\mathbf{r}}=\underbrace{\frac{\operatorname{erfc}(\omega \mathbf{r})}{\mathbf{r}}}_{S R}+\underbrace{\frac{\operatorname{erf}(\omega \mathbf{r})}{\mathbf{r}}}_{L R} .
$$


Paso 3 Escribir $E_{x}^{P B E 0}$ en componentes de $S R$ y $L R$ dependientes de $\omega$ :

$$
\begin{aligned}
E_{x}^{P B E 0} & =a E_{x}^{H F, S R}(\omega)+a E_{x}^{H F, L R}(\omega)+(1-a) E_{x}^{P B E, S R}(\omega) \\
& +(1-a) E_{x}^{P B E, L R}(\omega) \\
& =a E_{x}^{H F, S R}(\omega)+a E_{x}^{H F, L R}(\omega)+(1-a) E_{x}^{P B E, S R}(\omega) \\
& +E_{x}^{P B E, L R}(\omega)-a E_{x}^{P B E, L R}(\omega) .
\end{aligned}
$$

Paso 4 Por último, se considera que la contribución de los términos en rojo de la ecuación 3-64 son pequeños y tienden a cancelarse uno con otro, lo cual lleva a la expresión del funcional HSE06:

$$
E_{x c}^{H S E 06}=a E_{x}^{H F, S R}(\omega)+(1-a) E_{x}^{P B E, S R}(\omega)+E_{x}^{P B E, L R}(\omega)+E_{c}^{P B E}
$$

donde $\omega$ es un parámetro ajustable que controla la extensión de las interacciones de corto alcance.

\subsection{Estudio de la estructura electrónica de sólidos}

\subsubsection{Estructura cristalina}

En espacio real, un cristal es descrito en términos de un conjunto de vectores de red $\mathbf{R}$ dentro de una celda unitaria primitiva (CUP), los cuales conectan a todos los puntos equivalentes en el espacio, y definen la Red de Bravais [38]

$$
\mathbf{R}=n_{1} \mathbf{a}_{1}+n_{2} \mathbf{a}_{2}+n_{3} \mathbf{a}_{3}
$$

donde $n_{1}, n_{2}$ y $n_{3}$ son enteros arbitrarios, mientras que $\mathbf{a}_{1}, \mathbf{a}_{2}$ y $\mathbf{a}_{3}$ son los vectores de red primitivos, los cuales definen a la CUP como el volumen encerrado por estos tres:

$$
\Omega_{C U P}=\left|\mathbf{a}_{1} \cdot\left(\mathbf{a}_{2} \times \mathbf{a}_{3}\right)\right| .
$$

La utilidad de esta definición radica en que solo es necesario conocer las funciones en espacio real para toda $\mathbf{r}$ dentro de la CUP, ya que fuera de ésta sus valores son conocidos por una traslación R. Sin embargo, la descripción de los electrones de un cristal se basa en el espacio recíproco (al ocupar el Teorema de Bloch, siguiente sección), por lo que es necesario definir sus correspondientes vectores de red primitivos recíprocos: $\mathbf{b}_{1}, \mathbf{b}_{2} \mathbf{y}_{\mathbf{b}_{3}}$ que permiten construir una red análoga a la de Bravais pero en espacio recíproco, mediante el conjunto de vectores:

$$
\mathbf{G}=m_{1} \mathbf{b}_{1}+m_{2} \mathbf{b}_{2}+m_{3} \mathbf{b}_{3}
$$


donde el producto punto de cualquier vector $\mathbf{R}$ con cualquier vector $\mathbf{G}$ es

$$
\mathbf{R} \cdot \mathbf{G}=2 \pi l, l=n_{1} m_{1}+n_{2} m_{2}+n_{3} m_{3}
$$

donde $l$ siempre es un entero y cualquier función que posea la periodicidad de la red de Bravais puede ser escrita como

$$
f(\mathbf{r})=\sum_{\mathbf{G}} e^{i \mathbf{G} \cdot \mathbf{r}} f(\mathbf{G})
$$

con $f(\mathbf{G})$ siendo los componentes de la Trasformada de Fourier (TF). Debido a la periodicidad de la red, cualquier función necesita ser estudiada únicamente para $\mathbf{r}$ dentro de la CUP [38].

\subsubsection{Teorema de Bloch}

Para generar una función de onda que sea solución a la ecuación de Schrödinger de un sistema cristalino, se aprovecha la simetría traslacional del cristal a través del Teorema de Felix Bloch [63]. Cuando el hamiltoniano de una partícula presenta un potencial que posee la periodicidad translacional de la red de Bravais se tiene que

$$
V(\mathbf{r}+\mathbf{R})=V(\mathbf{r})
$$

por lo que las funciones de onda de una partícula tienen la misma simetría [38] y cada una puede ser escrita como un producto de un factor de fase (en azul) y una función $u_{\mathbf{k}}(\mathbf{r})[37,38]$

$$
\psi_{\mathbf{k}}(\mathbf{r})=e^{i \mathbf{k} \cdot \mathbf{r}} u_{\mathbf{k}}(\mathbf{r})
$$

donde la función $u_{\mathbf{k}}(\mathbf{r})$ tiene la periodicidad translacional completa de la red de Bravais [38]

$$
u_{\mathbf{k}}(\mathbf{r}+\mathbf{R})=u_{\mathbf{k}}(\mathbf{r})
$$

y puede ser expresada en un conjunto discreto de ondas planas ${ }^{\mathrm{m}}$ cuyos vectores de onda $\mathrm{G}$ son vectores de la red recíproca del cristal ${ }^{\mathrm{n}}$ :

$$
u_{\mathbf{k}}(\mathbf{r})=\sum_{\mathbf{G}} \alpha_{\mathbf{k}}(\mathbf{G}) e^{i \mathbf{G} \cdot \mathbf{r}} .
$$

Por lo que cada función de onda electrónica (ecuación 3-72) queda expresada al final como la siguiente suma de ondas planas [37,38]:

\footnotetext{
mla ecuación 3-74 es la expresión 3-70 en la cual la $f(\mathbf{G})$ es etiquetada por $\mathbf{k}$.

${ }^{n}$ descritos por la ecuación 3-68
} 


$$
\psi_{\mathbf{k}}(\mathbf{r})=\sum_{\mathbf{G}} \alpha_{\mathbf{k}}(\mathbf{G}) e^{i(\mathbf{k}+\mathbf{G}) \cdot \mathbf{r}}
$$

donde las funciones de onda $\psi_{\mathbf{k}}(\mathbf{r})$ son llamadas estados de Bloch $\mathbf{y} \mathbf{k}$ es además de una etiqueta para las funciones de onda, un vector definido en términos de los vectores de red recíproca y constantes complejas $k_{j}[38]$ :

$$
\mathbf{k}=k_{1} \mathbf{b}_{1}+k_{2} \mathbf{b}_{2}+k_{3} \mathbf{b}_{3}
$$

La gran ventaja de la ecuación 3-75 es que basta determinar aquellas funciones de onda $\psi_{\mathbf{k}}(\mathbf{r})$ dentro de la CUP del cristal, ya que por la traslación $\mathbf{R}$ se conectan los puntos equivalentes en toda la red de Bravais. Sin embargo, el precio a pagar es que el vector k puede tomar un número de valores $N=N_{1} N_{2} N_{3}$, siendo $N_{i}(j=1,2,3)$ el número de celdas unitarias en cada una de las direcciones dadas por los vectores $\mathbf{a}_{j}(j=1,2,3)$, por lo que bajo la concepción de que un sistema cristalino es una repetición infinita de la celda unitaria en las tres direcciones, entonces los valores de $\mathbf{k}$ se vuelven continuos. Por lo que es necesario definir una región análoga a la CUP pero en espacio recíproco, denominada zona de Brillouin [38].

\subsubsection{Método proyector de ondas aumentadas}

Tal y como se describió en la sección anterior, el Teorema de Bloch establece que cada una de las funciones de onda electrónicas puede ser representada mediante el desarrollo en un conjunto discreto de ondas planas [37]. Sin embargo, las funciones de onda en DFT presentan un comportamiento oscilatorio rápido en regiones cercanas al núcleo [37,64], por lo que requieren de un conjunto muy grande de ondas planas para representar a cada orbital en el desarrollo [37,65] y el cálculo se vuelve computacionalmente demandante [37]. Una estrategia para reducir este problema consiste en centrar la atención en la descripción de los electrones de valencia, tal como en el caso de la aproximación de estados electrónicos internos congelados, en la cual se asume que [64]:

1. los estados electrónicos internos se encuentran localizados dentro de regiones denominadas esferas de aumento $\mathrm{y}$

2. los estados electrónicos internos de átomos aislados no cambian por la formación de moléculas o sólidos.

Por lo que los estados electrónicos internos de KS son idénticos a estados electrónicos internos atómicos y únicamente los estados de valencia son tratados en los desarrollos [64]. Una forma de construir cada una de estas funciones de onda monoelectrónicas es utilizando el método del proyector de ondas aumentadas (projector augmented waves, $P A W$ ), propuesto por Blöchl en 1994 [66], cuyas cualidades son descritas en comparación con otros métodos en la siguiente tabla. 
Tabla 3-1: Similitudes del método PAW con OPW y PS

\section{OPW (Introducido por Herring [67])}

Aproximar la función propia de un cristal, $\left|\psi_{n}\right\rangle$ (estados de valencia y core), por medio de

una combinación lineal de ondas planas más una combinación lineal de funciones centradas sobre cada átomo [67].

$\downarrow$

Mediante un radio de corte $r_{c}$, se divide cada orbital KS, $\left|\psi_{n}\right\rangle$, en una parte suave, $\left|\tilde{\psi}_{n}\right\rangle$, y una oscilante localizada ${ }^{\mathrm{b}}[35]$.

$\downarrow$

Al final, $\left|\psi_{n}\right\rangle$ es obtenida mediante una transformación lineal a $\left|\tilde{\psi}_{n}\right\rangle[35]:\left|\psi_{n}\right\rangle=\hat{\mathcal{T}}\left|\tilde{\psi}_{n}\right\rangle$

\section{PAW (Propuesto por Blöchl [66])}

Únicamente los estados de valencia son tratados en el proceso auto-consistente (aproximación frozen-core) [64]

$\downarrow$

Representa cada $\left|\psi_{n}\right\rangle$ mediante una función auxiliar suave $\left|\tilde{\psi}_{n}\right\rangle$ y con ella se realiza el proceso variacional [64,66] $\downarrow$

Tiene la ventaja de que si es necesario, se puede recuperar $\left|\psi_{n}\right\rangle \quad:\left|\psi_{n}\right\rangle=\hat{\mathcal{T}}\left|\tilde{\psi}_{n}\right\rangle$

\section{PS (Trabajos de Phillips-Kleinman [68] y Antončík [69, 70])}

Calcular propiedades de los electrones de valencia en moléculas o sólidos,

ya que los estados de core permanecen casi sin cambios [35].

$\downarrow$

Representa cada $\left|\psi_{n}\right\rangle$ mediante una función auxiliar suave $\left|\tilde{\psi}_{n}\right\rangle$ tal que [35] $\epsilon_{n}\left|\tilde{\psi}_{n}\right\rangle=\epsilon_{n}\left|\psi_{n}\right\rangle$

$\downarrow$

$\left|\psi_{n}\right\rangle$ y $\left|\tilde{\psi}_{n}\right\rangle$ son idénticas a partir de un radio de corte $r_{c}$ en adelante [37].

$\downarrow$

Reemplaza el fuerte potencial de Coulomb del núcleo así como los efectos de los electrones de core, por un potencial iónico efectivo actuando sobre los electrones de valencia [35].

\footnotetext{
${ }^{a}$ La parte suave está deslocalizada en toda la celda unitaria
}

b Son funciones centradas en los núcleos, las cuales son contenidas en la forma explícita de un operador de transformación lineal $\hat{\mathcal{T}}$ 
El método PAW utiliza la aproximación de estados electrónicos internos congelados y la función de onda monoelectrónica de KS del $n$-ésimo estado, $\left|\psi_{n}\right\rangle$ [71] es obtenida mediante un operador de transformación lineal $\hat{\mathcal{T}}$ aplicado a una función de onda auxiliar suave $^{\tilde{\mathrm{n}}}\left|\tilde{\psi}_{n}\right\rangle[64-66,71]$ :

$$
\left|\psi_{n}\right\rangle=\hat{\mathcal{T}}\left|\tilde{\psi}_{n}\right\rangle
$$

donde $n$ es una etiqueta para el estado cuántico, el índice de banda, el punto $\mathbf{k}$ y el espín. De esta manera las ecuaciones de KS son expresadas como [64,65]:

$$
\hat{\mathcal{T}}^{\dagger} \hat{H} \hat{\mathcal{T}}\left|\tilde{\psi}_{n}\right\rangle=\epsilon_{n} \hat{\mathcal{T}}^{\dagger} \hat{\mathcal{T}}\left|\tilde{\psi}_{n}\right\rangle
$$

Cabe mencionar que las funciones $\left|\psi_{n}\right\rangle$ son suaves a partir de una cierta distancia al core, esto permite definir que el operador $\hat{\mathcal{T}}$ modifique a la función $\left|\tilde{\psi}_{n}\right\rangle$ solamente en las regiones cercanas a cada núcleo [64]:

$$
\hat{\mathcal{T}}=1+\sum_{a} \hat{\mathcal{T}}^{a}
$$

donde $a$ es un índice de sitio atómico y cada $\hat{\mathcal{T}}^{a}$ representa una transformación centrada en cada átomo, dentro de cada esfera de aumento (EA), definida como $\left|\mathbf{r}-\mathbf{R}^{a}\right|<r_{c}^{a}$, en la cual $r_{c}^{a}$ es un radio de corte seleccionado tal que no exista traslape entre las esferas de aumento [64]. En otras palabras, el operador $\hat{\mathcal{T}}$ tiene la tarea de modificar la $\left|\tilde{\psi}_{n}\right\rangle$ dentro de cada EA $\left(\left|\mathbf{r}-\mathbf{R}^{a}\right|<r_{c}^{a}\right)^{\circ}$, para que la función de onda resultante posea la estructura nodal correcta dentro de cada una de ellas [65]. Lo anterior se realiza mediante el desarrollo de $\left|\psi_{n}\right\rangle$ en el conjunto de funciones de base $\left\{\phi_{i}^{a}\right\}$ denominadas ondas parciales, todo esto únicamente dentro de cada EA. Análogamente se desarrolla $\left|\tilde{\psi}_{n}\right\rangle$ en el conjunto de funciones de base $\left\{\tilde{\phi}_{i}^{a}\right\}$ denominadas ondas parciales auxiliares y se requiere que $[64,65]$ :

$$
\left|\phi_{i}^{a}\right\rangle=\left(1+\hat{\mathcal{T}}^{a}\right)\left|\tilde{\phi}_{i}^{a}\right\rangle \quad \Leftrightarrow \quad \hat{\mathcal{T}}^{a}\left|\tilde{\phi}_{i}^{a}\right\rangle=\left|\phi_{i}^{a}\right\rangle-\left|\tilde{\phi}_{i}^{a}\right\rangle
$$

para toda $i^{\mathrm{p}}$, a. Esto define completamente a $\hat{\mathcal{T}}^{a}$, dados los conjuntos de base $\left\{\phi_{i}^{a}\right\} \mathrm{y}$ $\left\{\tilde{\phi}_{i}^{a}\right\}$ [65]. Para que $\hat{\mathcal{T}}^{a}$ no opere fuera de la EA, se debe cumplir que $\phi_{i}^{a}(\mathbf{r})^{\mathrm{q}}$ y su contraparte suave $\tilde{\phi}_{i}^{a}(\mathbf{r})$ sean idénticas fuera de la EA [64,65]:

$$
\forall a, \quad \phi_{i}^{a}(\mathbf{r})=\tilde{\phi}_{i}^{a}(\mathbf{r}), \quad \text { para } r>r_{c}^{a} .
$$

\footnotetext{
ñla cual debe ser idéntica a $\left|\psi_{n}\right\rangle$ a partir de un cierto radio de corte $r_{c}^{a}$ [64]

${ }^{\circ} \mathbf{R}^{a}$ es la posición del núcleo del sitio $a$ [65]

$\mathrm{p}_{i} \in a$. El índice $i$ hace referencia al índice $a$, momento angular $(\ell, m)$ y un índice adicional que permite diferenciar entre ondas parciales (auxiliares o no auxiliares) con los mismos números cuánticos de momento angular en el mismo sitio [65]

${ }^{\mathrm{q}} \phi_{i}^{a}(\mathbf{r})=\left\langle\mathbf{r} \mid \phi_{i}^{a}\right\rangle$
} 
Si las $\left|\tilde{\phi}_{i}^{a}\right\rangle$ constituyen un conjunto completo dentro de la EA en el sitio $a$, el desarrollo de $\left|\tilde{\Psi}_{n}\right\rangle$ en términos de esta base es ${ }^{\mathrm{r}}[64]$ :

$$
\left|\tilde{\psi}_{n}\right\rangle=\sum_{i \in a} P_{n i}^{a}\left|\tilde{\phi}_{i}^{a}\right\rangle, \quad \text { para }\left|\mathbf{r}-\mathbf{R}^{a}\right|<r_{c}^{a}
$$

donde $P_{n i}^{a}$ son los coeficientes del desarrollo que más adelante se especificarán. De acuerdo a la ecuación 3-80, $\left|\phi_{i}^{a}\right\rangle=\hat{\mathcal{T}}\left|\tilde{\phi}_{i}^{a}\right\rangle$ y desarrollo de $\left|\psi_{n}\right\rangle$ es [64]:

$$
\left|\psi_{n}\right\rangle=\hat{\mathcal{T}}\left|\tilde{\psi}_{n}\right\rangle=\sum_{i \in a} P_{n i}^{a}\left|\phi_{i}^{a}\right\rangle, \quad \text { para }\left|\mathbf{r}-\mathbf{R}^{a}\right|<r_{c}^{a}
$$

la cual posee los mismos coeficientes del desarrollo $P_{n i}^{a}$ de $\left|\tilde{\psi}_{n}\right\rangle$ [64].

Ya que se requiere que la transformación $\hat{\mathcal{T}}$ sea lineal, entonces los coeficientes $P_{n i}^{a}$ deben ser proporcionados por una proyección en cada EA [35, 64]:

$$
P_{n i}^{a}=\left\langle\tilde{p}_{i}^{a} \mid \tilde{\psi}_{n}\right\rangle=\int d \mathbf{r} \tilde{p}_{i}^{a *}\left(\mathbf{r}-\mathbf{R}^{a}\right) \tilde{\psi}_{n}(\mathbf{r})
$$

Como no hay traslape entre las EA, el desarrollo de un centro de $\left|\tilde{\psi}_{n}\right\rangle$ dentro de la EA en el sitio $a$,

$$
\left|\tilde{\psi}_{n}^{a}\right\rangle=\sum_{i \in a}\left|\tilde{\phi}_{i}^{a}\right\rangle\left\langle\tilde{p}_{i}^{a} \mid \tilde{\psi}_{n}\right\rangle
$$

debe reducirse a $\left|\tilde{\psi}_{n}\right\rangle$, esto es [64]:

$$
\left|\tilde{\psi}_{n}^{a}\right\rangle=\sum_{i \in a}\left|\tilde{\phi}_{i}^{a}\right\rangle\left\langle\tilde{p}_{i}^{a} \mid \tilde{\psi}_{n}\right\rangle=\left|\tilde{\psi}_{n}\right\rangle \Rightarrow \sum_{i \in a}\left|\tilde{\phi}_{i}^{a}\right\rangle\left\langle\tilde{p}_{i}^{a}\right|=1
$$

Al insertar la condición en rojo de la ecuación 3-86 entre el bra y ket rojos de la ecuación 3-85 se obtiene la condición de biortonormalidad

$$
\left\langle\tilde{p}_{i}^{a} \mid \tilde{\phi}_{i^{\prime}}^{a}\right\rangle=\delta_{i, i^{\prime}} \quad, \quad \text { para }\left|\mathbf{r}-\mathbf{R}^{a}\right|<r_{c}^{a} \quad .
$$

Esto significa que las funciones de proyección deben ser ortonormales a las ondas parciales suaves dentro de la EA [35, 64-66, 71].

Ahora multiplicamos $\hat{\mathcal{T}}^{a}$ por la izquierda en la relación completeness (término de la izquierda de la tercera igualdad de la ecuación 3-86) para obtener la cara de $\hat{\mathcal{T}}^{a}[64]^{\mathrm{s}}$ :

$$
\hat{\mathcal{T}}^{a}=\sum_{i \in a} \hat{\mathcal{T}}^{a}\left|\tilde{\phi}_{i}^{a}\right\rangle\left\langle\tilde { p } _ { i } ^ { a } \left|=\sum_{i \in a}\left(\left|\phi_{i}^{a}\right\rangle-\left|\tilde{\phi}_{i}^{a}\right\rangle\right)\left\langle\tilde{p}_{i}^{a}\right|\right.\right.
$$

${ }^{\mathrm{r}}$ Con $i \in a$ se indica que las ondas parciales pertenecen al sitio $a$ [65].

${ }^{\mathrm{s}}$ Cabe mencionar que las ecuaciones 3-80 a 3-88 son expresiones en cada EA del sistema bajo estudio. 
Con esta última expresión podemos escribir el operador $\hat{\mathcal{T}}$ de la ecuación 3-79 [35, 64-66, 71]. :

$$
\hat{\mathcal{T}}=1+\sum_{a} \sum_{i \in a}\left(\left|\phi_{i}^{a}\right\rangle-\left|\tilde{\phi}_{i}^{a}\right\rangle\right)\left\langle\tilde{p}_{i}^{a}\right|
$$

Finalmente podemos obtener todas las $\left|\psi_{n}\right\rangle$ de valencia a partir de la transformación lineal $\hat{\mathcal{T}}$ [64]:

$$
\begin{aligned}
\left|\psi_{n}\right\rangle & =\hat{\mathcal{T}}\left|\tilde{\psi}_{n}\right\rangle \\
& =\left|\tilde{\psi}_{n}\right\rangle+\sum_{a} \sum_{i \in a}\left(\left|\phi_{i}^{a}\right\rangle-\left|\tilde{\phi}_{i}^{a}\right\rangle\right)\left\langle\tilde{p}_{i}^{a} \mid \tilde{\psi}_{n}\right\rangle \\
& =\left|\tilde{\psi}_{n}\right\rangle-\sum_{a} \sum_{i \in a}\left|\tilde{\phi}_{i}^{a}\right\rangle\left\langle\tilde{p}_{i}^{a} \mid \tilde{\psi}_{n}\right\rangle+\sum_{a} \sum_{i \in a}\left|\phi_{i}^{a}\right\rangle\left\langle\tilde{p}_{i}^{a} \mid \tilde{\psi}_{n}\right\rangle \\
& =\left|\tilde{\psi}_{n}\right\rangle-\sum_{a} \sum_{i \in a}\left|\tilde{\phi}_{i}^{a}\right\rangle P_{n i}^{a}+\sum_{a} \sum_{i \in a}\left|\phi_{i}^{a}\right\rangle P_{n i}^{a}
\end{aligned}
$$

Como $\left|\phi_{i}^{a}\right\rangle$ y $\left|\tilde{\phi}_{i}^{a}\right\rangle$ son iguales fuera de EA, la ecuación 3-90 consiste en reemplazar el comportamiento suave de $\left|\tilde{\phi}_{i}^{a}\right\rangle$ dentro de cada EA (mediante los conjuntos de base $\left|\tilde{\phi}_{i}^{a}\right\rangle$ ) por un comportamiento oscilatorio (mediante los conjuntos de base $\left|\phi_{i}^{a}\right\rangle$ ) para obtener $\left|\phi_{i}^{a}\right\rangle$.

En resumen, el método PAW brinda la siguiente solución al problema de desarrollar cada orbital de KS con un conjunto muy grande de ondas planas, mediante estas ideas:

1) Utilizar la aproximación de estados electrónicos internos congelados para involucrar en el proceso auto-consistente únicamente a los orbitales de KS de valencia. Esto significa que los estados electrónicos internos permanecen sin cambios durante todo el proceso de cálculo.

2) Representar cada orbital de KS $\left|\psi_{n}\right\rangle$ con una función de onda auxiliar suave $\left|\tilde{\psi}_{n}\right\rangle$, la cual, debido a su comportamiento suave, requiere un menor número de funciones de ondas planas para su desarrollo (tal como en el caso de PS).

3) La $\left|\tilde{\psi}_{n}\right\rangle$ obtenida al final del proceso variacional puede ser utilizada para recuperar la $\left|\psi_{n}\right\rangle$ mediante una transformación lineal (tal como en el caso de OPW).

El primer punto trae como consecuencia que la demanda computacional disminuye al incluir menor número de $\left|\psi_{n}\right\rangle$ en el proceso auto-consistente; mientras que el segundo punto también disminuye el costo computacional pero esto es debido a que la función a desarrollar en ondas planas, debido a su comportamiento suave, requiere un menor número de ondas planas para su desarrollo. El tercer punto describe la gran ventaja de 
recuperar $\left|\psi_{n}\right\rangle$ y con ello la información de interés contenida en ella, sin la necesidad de involucrarla en el proceso auto-consistente. 


\section{Capítulo

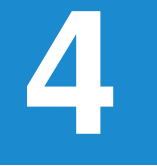 \\ HIPÓTESIS}

La sustitución de algunos átomos de $\mathrm{Cu}$ por $\mathrm{M}=\mathrm{Ni}$, Co o $\mathrm{Ti}$, en la red cristalina del $\mathrm{Li}_{2} \mathrm{CuO}_{2}$, modificará la participación redox de oxígeno en cada caso y esto puede ser determinado a través de la blandura local y con ello es posible proponer sustituciones que mejoren el desempeño de $\mathrm{Li}_{2} \mathrm{CuO}_{2}$ como material catódico. 


\section{Capítulo}

5 Objetivos

\subsection{Objetivo general}

Determinar la participación redox de oxígeno en $\mathrm{Li}_{2} \mathrm{CuO}_{2}$ y sistemas tipo $\mathrm{Li}_{2} \mathrm{Cu}_{1-x} \mathrm{M}_{x} \mathrm{O}_{2}$ a través de la blandura local y la densidad de estados proyectada, para establecer el efecto sobre dichas propiedades, que tiene la sustitución de algunos átomos de $\mathrm{Cu}$ por $\mathrm{Co}, \mathrm{Ni}$ o $\mathrm{Ti}$, en la estructura cristalina de $\mathrm{Li}_{2} \mathrm{CuO}_{2}$.

\subsection{Objetivos particulares}

1. Obtener las estructuras relajadas de los sistemas $\mathrm{Li}_{2} \mathrm{CuO}_{2}$ y $\mathrm{LiCuO}_{2}$ con la aproximación $\mathrm{PBE}+\mathrm{U}[60]$.

2. Determinar el efecto de sustitución de átomos de $\mathrm{Cu}$ por $\mathrm{M}(\mathrm{M}=\mathrm{Co}, \mathrm{Ni}$ o $\mathrm{Ti})$ en la estructura de $\mathrm{Li}_{2} \mathrm{CuO}_{2}$, mediante las estructuras relajadas de los sistemas $\mathrm{Li}_{2} \mathrm{Cu}_{1-x} \mathrm{M}_{x} \mathrm{O}_{2}$ con la aproximación $\mathrm{PBE}+\mathrm{U}$.

3. Estimar el voltaje promedio de inserción de litio.

4. Conocer la participación redox de oxígeno en $\mathrm{Li}_{2} \mathrm{CuO}_{2}, \mathrm{LiCuO}_{2}$ y Li $\mathrm{Cu}_{1-x} \mathrm{M}_{x} \mathrm{O}_{2}$, a partir de la DOS-p.

5. Proponer la gráfica de la blandura local para establecer la participación redox de oxígeno en cada sistema. 


\section{Capítulo}

\section{Metodología}

Todos los cálculos fueron realizados en el marco de la DFT [40], mediante la resolución de las ecuaciones de Kohn-Sham [41] bajo condiciones periódicas en el programa VASP (Vienna Ab Initio Simulation Package) [72-75] versión 5.4.1 y utilizando el método PAW para tratar las interacciones electrones internos-electrones de valencia [66, 71]. El programa utilizado para visualización de los resultados estructurales y la blandura local $(s(\mathbf{r}))$ fue VESTA (Visualization for Electronic and STructural Analysis) [76] versión 3.3.2. Cabe mencionar que las aproximaciones al funcional de intercambio-correlación utilizadas en este trabajo, son especificadas en cada sección.

\subsection{Sistemas $\mathrm{Li}_{2} \mathrm{CuO}_{2}$ y $\mathrm{LiCuO}_{2}$}

La parte inicial de este trabajo consistió del estudio de los sistemas $\mathrm{Li}_{2} \mathrm{CuO}_{2} \mathrm{y} \mathrm{LiCuO}_{2}$, por lo que se utilizó la base de datos ICSD (Inorganic Crystal Structure Database, https://icsd.fizkarlsruhe.de/index.xhtml), de la cual se obtuvieron las celdas unitarias experimentales de $\mathrm{Li}_{2} \mathrm{CuO}_{2}$ [77] y $\mathrm{LiCuO}_{2}$ [78].

Previo a la relajación estructural de los sistemas, se realizaron pruebas de convergencia a geometría fija para determinar el número de puntos $k$ y la energía de corte para el desarrollo en ondas planas, buscando una convergencia en la energía menor a $1 \mathrm{meV}$. Todo esto se realizó utilizando el funcional PBE [45, 46, 71] como aproximación al funcional de intercambio-correlación. El muestreo de puntos $k$ y la energía de corte con los que se alcanzó la convergencia deseada se muestran en la Tabla 6-1. 
Tabla 6-1: Malla de puntos $k$ y energía de corte obtenidas de las pruebas de convergencia en la energía

\begin{tabular}{ccc}
\hline Sistema & Malla de puntos $k$ & Energía de corte (eV) \\
$\mathrm{LiCuO}_{2}$ & $6 \times 12 \times 6$ & 900 \\
$\mathrm{Li}_{2} \mathrm{CuO}_{2}$ & $12 \times 12 \times 4$ & 900 \\
\hline \hline
\end{tabular}

Una vez establecidos el muestreo de puntos $k$ así como la energía de corte en cada caso, se llevó a cabo la relajación de ambos sistemas, con la restricción de mantener la simetría de la celda unitaria y utilizando el funcional PBE. El criterio de paro en la optimización de la posición de los iones se fijó hasta que las fuerzas sobre estos fueran $<0.01 \mathrm{eV} / \AA$.

Las estructuras relajadas de $\mathrm{Li}_{2} \mathrm{CuO}_{2}$ y $\mathrm{LiCuO}_{2}$ con el funcional PBE fueron utilizadas como entrada para relajar con la aproximación $\mathrm{PBE}+\mathrm{U}$ en el esquema de Dudarev et al. [60], utilizando un valor de $U_{\text {efectivo }}=6 \mathrm{eV}$ para los átomos de cobre [79]. Previo a la relajación de estos sistemas, se realizaron pruebas de magnetización ${ }^{a}$ a geometría fija, que ayudaron a proponer el estado de magnetización atómica inicial de menor energía, para los átomos de $\mathrm{Cu}$ de cada sistema. Posteriormente, se llevó a cabo la relajación de cada sistema con un criterio de paro en las fuerzas $<0.01 \mathrm{eV} / \AA$, así como la malla de puntos $k$ y la energía de corte de la Tabla 6-1. Después de obtener las estructuras relajadas, nuevamente se realizaron pruebas de magnetización, de las cuales se seleccionó el estado con la magnetización de menor energía de cada sistema, a los cuales posteriormente se les determinó la densidad de estados proyectada, $D O S-p$, blandura local, $s(\mathbf{r})$, así como el voltaje teórico de inserción de litio.

\subsection{Sistemas del tipo $\mathrm{Li}_{2} \mathrm{Cu}_{0.5} \mathrm{M}_{\mathrm{C}} \mathrm{O}$}

De la Sección 2.3, podemos notar que la sustitución de átomos de $\mathrm{Cu}$ por Co o $\mathrm{Ni}$ en la estructura cristalina de $\mathrm{Li}_{2} \mathrm{CuO}_{2}$, generó sistemas que mostraron valores mayores de capacidad (cantidad de carga almacenada en la celda, expresada en amperio/hora) respecto de $\mathrm{Li}_{2} \mathrm{CuO}_{2}$. Por otro lado, en el contexto de materiales catódicos laminares abundantes en litio, el efecto de incluir Ti en la estructura del material, teóricamente, disminuye la pérdida de oxígeno ${ }^{b}$ en comparación con otros metales de transición tales como Co y Mn [80]. Estos fueron los motivos para estudiar el efecto de la sustitución de átomos de Cu por átomos de $\mathrm{Co}, \mathrm{Ni}$ o Ti, en la estructura electrónica de $\mathrm{Li}_{2} \mathrm{CuO}_{2}$, a partir de su celda unitaria relajada con $\mathrm{PBE}+\mathrm{U}$, la cual se utilizó como plantilla para construir los sistemas $\mathrm{Li}_{2} \mathrm{Cu}_{0.5} \mathrm{M}_{0.5} \mathrm{O}_{2}$

\footnotetext{
${ }^{a}$ En estas pruebas se propuso un momento magnético inicial en cada átomo de cada sistema, cada valor de momento magnético fue propuesto en base a la teoría del campo cristalino.

${ }^{\text {b} E l ~ s i s t e m a ~ q u e ~ c o n t e n i ́ a ~ t i t a n i o ~ e n ~ s u ~ e s t r u c t u r a ~ c r i s t a l i n a, ~ p r e s e n t o ́ ~ l a ~ e n e r g i ́ a ~ m a ́ s ~ a l t a ~ p a r a ~ f o r m a r ~ v a c a n-~}$ cias de oxígeno, lo cual implica menor participación redox de oxígeno, efecto que es no deseado en estos sistemas.
} 
$(\mathrm{M}=\mathrm{Co}$,Ni o Ti), sustituyendo un átomo de Cu por M (Figura 6-1). Los sistemas fueron tratados con la aproximación PBE+U bajo el mismo esquema que $\mathrm{Li}_{2} \mathrm{CuO}_{2}$ en puntos $k$, energía de corte y criterio de paro en las fuerzas (Sección 6.1), utilizando los valores de $U_{\text {efectivo }}$ de la Tabla 6-2 y también realizando pruebas de magnetización antes y después de la relajación estructural de cada sistema.

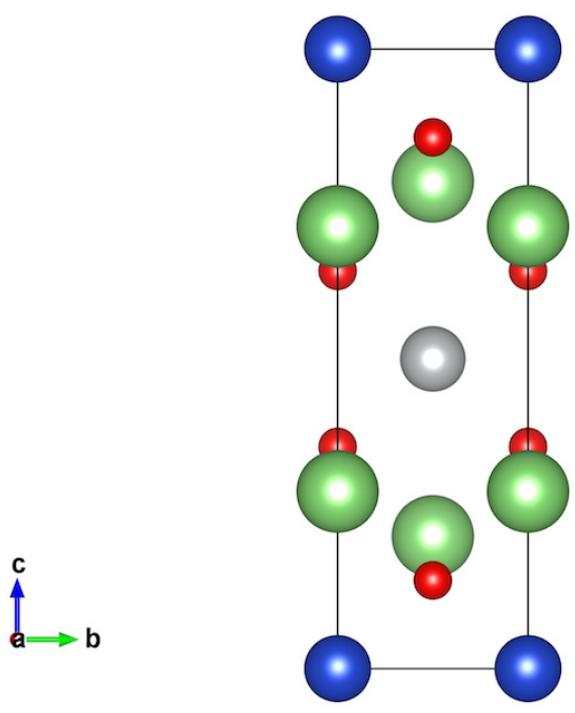

Figura 6-1: Celda unitaria de los sistemas $\mathrm{Li}_{2} \mathrm{Cu}_{0.5} \mathrm{M}_{0.5} \mathrm{O}_{2}$. Las esferas color verde representan los átomos de litio, las azules, átomos de cobre, las rojas oxígenos y la gris representa al átomo $\mathrm{M}=\mathrm{Co}$, Ni o Ti.

Tabla 6-2: Valores de $U_{\text {efectivo }}$ para los metales de transición utilizados en este trabajo

\begin{tabular}{ccc}
\hline \hline Metal de transición & $U_{\text {efectivo }}(\mathrm{eV})$ & Referencia \\
$\mathbf{C u}$ & 6.00 & {$[79]$} \\
$\mathbf{C o}$ & 5.10 & {$[81]$} \\
$\mathbf{N i}$ & 5.96 & {$[82]$} \\
$\mathbf{T i}$ & 4.20 & {$[83]$} \\
\hline \hline
\end{tabular}

\subsection{Cálcullo de la densidad de estados proyectada y la blandura local}

A las celdas unitarias de $\mathrm{Li}_{2} \mathrm{CuO}_{2}, \mathrm{LiCuO}_{2}$ y $\mathrm{Li}_{2} \mathrm{Cu}_{0.5} \mathrm{M}_{0.5} \mathrm{O}_{2}$ relajadas con $\mathrm{PBE}+\mathrm{U}$, se les determinó la DOS-p por tipo de átomo y por ambiente químico, mediante un cálculo a geometría fija para cada sistema. Con la finalidad de hacer una comparación del desempeño de la aproximación $\mathrm{PBE}+\mathrm{U}$ en la descripción de la densidad de estados y la blandura local, lo anterior fue realizado con dos aproximaciones al funcional de intercambiocorrelación: PBE+U y HSE06 [61]. Se utilizó la misma malla de puntos $k$ y energía de corte 
que $\mathrm{Li}_{2} \mathrm{CuO}_{2}$, así como el $U_{\text {efectivo }}$ de la Tabla 6-2 (en el caso de PBE+U) para cada sistema.

La blandura local, $s(\mathbf{r})$, es un coeficiente de respuesta definido dentro del formalismo gran canónico de la DFT, introducido por Yang y Parr [84] y generalizado por Cohen et al. [85]. Este concepto ha sido útil para racionalizar la reactividad química relacionada en procesos de transferencia electrónica en moléculas [86], sólidos y superficies [87-90]. Su definición es

$$
s(\mathbf{r}) \equiv\left(\frac{\partial \rho(\mathbf{r}, \mu)}{\partial \mu}\right)_{v(\mathbf{r})}
$$

donde $\rho(\mathbf{r})$ es la densidad electrónica, $\mu$ es el potencial químico y $v(\mathbf{r})$ representa el potencial externo. En un sólido, la densidad electrónica es descrita en términos de la densidad de estados local, $g(\mathbf{r}, \epsilon, \mu)$

$$
\rho(\mathbf{r}, \mu)=\int^{\mu} g(\mathbf{r}, \varepsilon, \mu) d \varepsilon
$$

En esta ecuación, siguiendo a Cohen et al. [85], se enfatiza la dependencia de la densidad de estados local con respecto a el potencial químico, por lo que un cambio finito en la densidad electrónica puede ser expresado en términos de un cambio en el potencial químico como

$$
\rho\left(\mathbf{r}, \mu_{0}+\Delta \mu\right)=\int^{\mu_{0}+\Delta \mu} g\left(\mathbf{r}, \varepsilon, \mu_{0}+\Delta \mu\right) d \varepsilon
$$

En esta expresión $\mu_{0}$ es un potencial químico de referencia. Desarrollando $g(\mathbf{r}, \varepsilon, \mu)$ con una serie de Taylor hasta primer orden en $\mu$, alrededor del potencial químico de referencia, se puede obtener una expresión a primer orden, para la diferencia de densidades electrónicas en la forma:

$$
\rho\left(\mathbf{r}, \mu_{0}+\Delta \mu\right)-\rho\left(\mathbf{r}, \mu_{0}\right) \approx\left[\frac{1}{\Delta \mu} \int_{\mu_{0}}^{\mu_{0}+\Delta \mu} g\left(\mathbf{r}, \varepsilon, \mu_{0}\right) d \varepsilon+\int^{\mu_{0}+\Delta \mu}\left(\frac{\partial g(\mathbf{r}, \varepsilon, \mu)}{\partial \mu}\right)_{\mu=\mu_{0}} d \varepsilon\right] \Delta \mu
$$


Donde la cantidad dentro de los corchetes cuadrados corresponde a la primera derivada de la densidad electrónica con respecto al potencial químico, en una aproximación de diferencias finitas

$$
s(\mathbf{r}) \approx \frac{1}{\Delta \mu} \int_{\mu_{0}}^{\mu_{0}+\Delta \mu} g\left(\mathbf{r}, \varepsilon, \mu_{0}\right) d \varepsilon+\int^{\mu_{0}+\Delta \mu}\left(\frac{\partial g(\mathbf{r}, \varepsilon, \mu)}{\partial \mu}\right)_{v(\mathbf{r}), \mu=\mu_{0}} d \varepsilon
$$

El signo de $\Delta \mu$ depende de si el sistema acepta o dona electrones ${ }^{\mathrm{c}}$. El segundo término en la ecuación de arriba representa la relajación de la densidad de estados local cuando el potencial químico es modificado. Si esta relajación se desprecia, obtenemos una expresión para la blandura local dentro de la aproximación de banda rígida [90]:

$$
s(\mathbf{r}) \approx \frac{1}{\Delta \mu} \int_{\mu_{0}}^{\mu_{0}+\Delta \mu} g\left(\mathbf{r}, \varepsilon, \mu_{0}\right) d \varepsilon
$$

Se han realizado esfuerzos para ir más allá de esta aproximación [85,89,91], sin embargo, en este trabajo se aplicó la ecuación 6-6 para estimar la $s(\mathbf{r})$, donde $\Delta \mu$ se fijó mediante la condición:

$$
\int_{\Omega} \int_{\varepsilon_{f}}^{\varepsilon_{f}+\Delta \mu} g\left(\mathbf{r}, \varepsilon, \mu_{0}\right) d \varepsilon d \mathbf{r}=1.0
$$

y debido a que se considera la oxidación del sólido, el signo de $\Delta \mu$ es negativo, porque los valores de energía que se consideran son aquellos correspondientes a estados ocupados. La integral sobre $\mathbf{r}$ es evaluada en el volumen de la celda unitaria, $\Omega$. Esta condición corresponde, dentro del esquema de diferencias finitas, a la idea de considerar la transferencia de un electrón. Una condición similar ha sido aplicada en otros contextos [23].

La $s(\mathbf{r})$ es una función de tres variables, por lo tanto una manera de visualizarla es graficar una superficie, en la cual la función $s(\mathbf{r})$ tiene un valor constante. Primero se determinó

\footnotetext{
${ }^{\mathrm{c}}$ En caso de que el sistema acepte electrones el signo de $\Delta \mu$ será positivo, porque la integral se realizará considerando estados más altos en energía que el nivel de Fermi. Por otro lado, en caso de que el sistema pierda electrones, el signo de $\Delta \mu$ será negativo, porque la integral se realizará considerando estados de menor energía que el nivel de Fermi.
} 
para cada sistema el valor de $\Delta \mu$ que establece el intervalo de energía respecto al nivel de Fermi, en el cual se cumple la condición de la ecuación 6-7, lo que representa la pérdida de un electrón en un proceso de transferencia de carga. Mediante esta información, fue posible graficar la $s(\mathbf{r})$ e identificar los sitios susceptibles a ser oxidados bajo este primer proceso de carga. Lo anterior fue realizado con cinco aproximaciones al funcional de intercambio-correlación: PBE [45, 46,71], PBEsol [92], SCAN [93], PBE+U (bajo el esquema de Dudarev y colaboradores [60]) y HSE06 [61]. Se utilizó la misma malla de puntos $k$, energía de corte y $U_{\text {efectivo }}$ para cada sistema, que para la obtención de la DOS-p. La obtención de la $s(\mathbf{r})$ con estas cinco aproximaciones, fue con el objetivo de conocer la sensibilidad de este índice de reactividad ante diferentes aproximaciones al funcional de intercambio-correlación.

\subsection{Cálculo del voltaje teórico promedio de inserción de litio}

El voltaje de circuito abierto obtenido entre los dos electrodos de la celda electroquímica de ion litio (ánodo y cátodo), depende de la diferencia de potencial químico de Li entre los mismos [5]:

$$
V(x)=-\frac{\mu_{L i}^{c a ́ t o d o}(x)-\mu_{L i}^{a ́ n o d o}}{z F}
$$

donde $F$ es la constante de Faraday y $z$ es el número de cargas transportadas por el litio en el electrolito (es adimensional) . Al asumir que el ánodo está compuesto únicamente por litio metálico, entonces el voltaje de la celda dependerá sólo del potencial químico del cátodo $^{\mathrm{d}}$, por lo que a medida de que se intercale más litio dentro del cátodo, su potencial químico de Li incrementa y el voltaje de la celda disminuye. Al graficar el voltaje de la celda en función del contenido de litio en el cátodo, se obtiene una curva de intercalación, la cual ha sido obtenida a partir de medidas experimentales en materiales catódicos basados en óxidos de metales de transición $\left(\mathrm{Li}_{x} \mathrm{MO}_{2}\right.$, donde $x$ es la composición de litio en el cátodo $\mathrm{y}$ $\mathrm{M}=$ Metal de transición). Sin embargo, desde el punto de vista de los cálculos de estructura electrónica es demandante obtener una curva de intercalación debido a: 1) representar la curva de intercalación en un rango continuo de composición de litio, $x$, requiere evaluar un número infinito de composiciones de litio en el cátodo ${ }^{\mathrm{e}}, 2$ ) la construcción de la celda unitaria del cátodo a cierta composición de litio, podría demandar crecer la celda unitaria original a una dimensión intratable ${ }^{\mathrm{f}}$ para los métodos de estructura electrónica y 3) la existencia de múltiples configuraciones para representar una misma composición de litio en el material catódico, lo que dificulta el proceso de obtención de la curva de intercalación.

\footnotetext{
${ }^{\mathrm{d}}$ Se suprime la dependencia en la concentración de Li en el ánodo porque el potencial químico es constante en una sustancia pura tal como en este caso.

${ }^{e} \mathrm{O}$ en su defecto construir la curva con valores discretos de composición de litio, lo cual puede ser acompañado por la razón 3) de este párrafo.

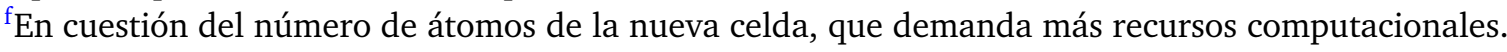


Estas dificultades pueden ser evitadas si se calcula el voltaje de intercalación de litio en una forma promedio, tal y como lo propuso Aydinol y colaboradores [5].

En un ánodo de litio metálico $\left(\mathrm{Li}^{0}\right)$, el potencial químico de Li es constante e igual a la energía de Gibbs de Li, por lo que el trabajo eléctrico, $W_{e}$, obtenido por la descarga entre $\mathrm{Li}_{x_{1}} \mathrm{MO}_{2}$ y Li $x_{x_{2}} \mathrm{MO}_{2}\left(x_{2}>x_{1}\right)$ es la integral del voltaje por la carga total $q_{t o t}{ }^{g}$ transferida desde la composición inicial de litio $x_{1}$ a la composición final de litio $x_{2}$ [5]:

$$
W_{e}=\int_{0}^{q_{t o t}} V(x) d q=-\int_{0}^{q_{t o t}} \frac{\mu_{L i}^{I C}(x)-\mu_{L i}^{0}}{e} d q
$$

donde $\mu_{L i}^{I C}(x)$ es el potencial químico de Li (por átomo) en el cátodo (compuesto de intercalación, IC), $\mu_{L i}^{0}$ es el potencial químico en litio metálico y $e$ es la carga elemental (expresada en coulombs, C). Al considerar que toda la carga desplazada es debido a Li, $d q=e d x$, entonces la ecuación 6-9 se transforma en [5]:

$$
\begin{aligned}
W_{e} & =-\int_{x_{1}}^{x_{2}}\left[\mu_{L i}^{I C}(x)-\mu_{L i}^{0}\right] d x_{L i} \\
& =-\left[G_{L i_{x_{2}} M O_{2}}-G_{L i_{x_{1}} M O_{2}}-\left(x_{2}-x_{1}\right) G_{L i}\right] \\
& \equiv-\Delta G_{r}
\end{aligned}
$$

Este $\Delta G_{r}$ corresponde a la siguiente reacción general de inserción de litio:

$$
L i_{x_{1}} M O_{2(s)}+\left(x_{2}-x_{1}\right) L i_{(s)}^{0} \longrightarrow L i_{x_{2}} M O_{2(s)}
$$

donde $M$ representa un átomo de metal de transición. Por lo que el voltaje promedio ${ }^{\mathrm{h}}$ es [5]:

$$
\bar{V}=-\frac{\Delta G_{r}}{q_{t o t}}=-\frac{\Delta G_{r}}{\left(x_{2}-x_{1}\right) e}=-\frac{\Delta G_{r} N_{A}}{\left(x_{2}-x_{1}\right) N_{A} e}=-\frac{\Delta G_{r}^{M}}{\left(x_{2}-x_{1}\right) F}
$$

Donde $\bar{V}$ representa el voltaje teórico promedio de inserción de litio, $N_{A}$ es el número de Avogadro, $\Delta G_{r}^{M}$ corresponde al cambio en la energía de Gibbs molar de la reacción, $q_{t o t}$ es la carga total transferida y $F$ es la constante de Faraday (96485.3383 C/mol) [3]. El $\Delta G_{r}$ es definido como

$$
\Delta G_{r} \equiv \Delta E_{r}+P \Delta V_{r}-T \Delta S_{r}
$$

En esta expresión $\Delta E_{r}$ es el cambio en la energía total de reacción, calculada en el marco de la DFT, $P$ es la presión, $\Delta V_{r}$ y $\Delta S_{r}$ son los cambios en el volumen y entropía de reacción, respectivamente. Considerando que:

${ }^{\mathrm{g}} q_{t o t}=e\left(x_{2}-x_{1}\right)$

hSe llama voltaje promedio porque los límites de integración son considerados a ser valores enteros, por lo que la diferencia $\left(x_{2}-x_{1}\right)$ resulta ser un número entero. 
$\star \Delta E_{r}$ es del orden de $3-4 \mathrm{eV}$ por molécula $[5,6,94]$,

$\star P \Delta V_{r}$ es del orden de $10^{-5} \mathrm{eV}[5,6,94,95] \mathrm{y}$

$\star T \Delta S_{r}$ del orden de la energía térmica $k_{B} T$ [95].

Podemos hacer la siguiente aproximación a $T=0 \mathrm{~K}$

$$
\Delta G_{r} \approx \Delta E_{r}
$$

es decir, el $\Delta E_{r}$ está asociado a la diferencia entre las energías totales de reactivos y productos.

La reacción 6-11 escrita para $\mathrm{Li}_{2} \mathrm{CuO}_{2}$ y Li $\mathrm{Cu}_{0.5} \mathrm{M}_{0.5} \mathrm{O}_{2}$ es:

$$
\mathrm{LiCu}_{0.5} \mathrm{M}_{0.5} \mathrm{O}_{2(s)}+1 \mathrm{Li} i_{(s)}^{0} \longrightarrow \mathrm{Li}_{2} \mathrm{Cu} u_{0.5} \mathrm{M}_{0.5} \mathrm{O}_{2(s)}
$$

en la cual $M$ representa $\mathrm{Cu}$, Co, $\mathrm{Ni}$ o Ti, en el caso de $\mathrm{Li}_{2} \mathrm{CuO}_{2}, \mathrm{Li}_{2} \mathrm{Cu}_{0.5} \mathrm{Co}_{0.5} \mathrm{O}_{2}, \mathrm{Li}_{2} \mathrm{Cu}_{0.5} \mathrm{Ni}_{0.5} \mathrm{O}_{2}$ y $\mathrm{Li}_{2} \mathrm{Cu}_{0.5} \mathrm{Ti}_{0.5} \mathrm{O}_{2}$ respectivamente. La reacción correspondiente al sistema $\mathrm{LiCuO}_{2}$, de forma análoga, queda como:

$$
\mathrm{CuO}_{2(s)}+1 \mathrm{Li}_{(s)}^{0} \longrightarrow \mathrm{LiCuO}_{2(s)}
$$

Estas reacciones fueron consideradas en el cálculo del $\Delta E_{r}$.

\subsection{Sistemas del tipo $\mathrm{Li}_{2} \mathrm{Cu}_{0.75} \mathrm{M}_{0.25} \mathrm{O}_{2}$}

Existe la posibilidad de que experimentalmente, la sustitución de algunos átomos de $\mathrm{Cu}$ en $\mathrm{Li}_{2} \mathrm{CuO}_{2}$ con otro metal de transición $\mathrm{M}$ (por ejemplo, $\mathrm{M}=\mathrm{Co}$, Ni o Ti), genere un material donde algunas láminas de metal de transición contengan a ambos metales de transición ${ }^{\mathrm{i}}$, por lo que resulta de interés conocer el comportamiento de la DOS-p así como el de la $s(\mathbf{r})$ en este tipo de sistemas. Por este motivo, se construyeron los sistemas de fórmula $\mathrm{Li}_{2} \mathrm{Cu}_{0.75} \mathrm{M}_{0.25} \mathrm{O}_{2}$ ( $\mathrm{M}=\mathrm{Co}$, Ni o Ti), (Figura 6-2) $)^{\mathrm{j}}$, donde una de las láminas de metal de transición está formada por $\mathrm{Cu}$ y M. La posición de los iones de estos sistemas fue relajada con $\mathrm{PBE}+\mathrm{U}$, bajo las mismas condiciones utilizadas para los sistemas $\mathrm{Li}_{2} \mathrm{Cu}_{0.5} \mathrm{M}_{0.5} \mathrm{O}_{2}$.

${ }^{\mathrm{i}}$ Como en el caso de $\mathrm{Li}_{2} \mathrm{Cu}_{0.5} \mathrm{Ni}_{0.5} \mathrm{O}_{2} \quad[16]$

${ }^{j} \mathrm{~A}$ partir de la celda unitaria de $\mathrm{Li}_{2} \mathrm{CuO}_{2}$ obtenida de la relajación con $\mathrm{PBE}+\mathrm{U}$, la cual se replicó en dirección b. 


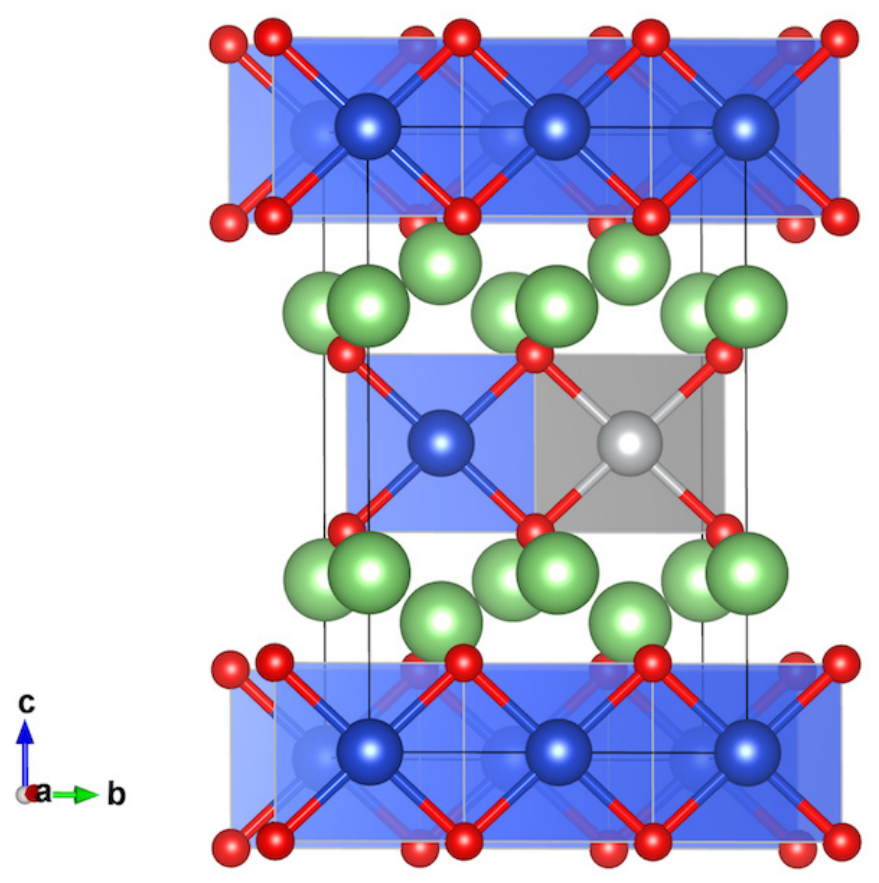

Figura 6-2: Celda unitaria del sistema $\mathrm{Li}_{2} \mathrm{Cu}_{0.75} \mathrm{M}_{0.25} \mathrm{O}_{2}$. Las esferas color verde representan los átomos de litio, las azules, átomos de cobre, las rojas oxígenos y la gris representa al átomo $\mathrm{M}=\mathrm{Co}, \mathrm{Ni}$ o $\mathrm{Ti}$.

Al igual que para los sistemas $\mathrm{Li}_{2} \mathrm{Cu}_{0.5} \mathrm{M}_{0.5} \mathrm{O}_{2}$, a los sistemas $\mathrm{Li}_{2} \mathrm{Cu}_{0.75} \mathrm{M}_{0.25} \mathrm{O}_{2}(\mathrm{M}=\mathrm{Co}, \mathrm{Ni}$ o Ti), se les determinó su DOS-p y $s(\mathbf{r})$ utilizando el funcional HSE06, con la misma malla de puntos $k$ y energía de corte que los cálculos con $\mathrm{PBE}+\mathrm{U}$. 


\section{Capítulo}

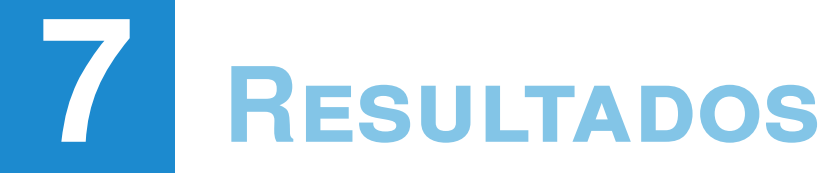

\subsection{Parámetros estructurales de $\mathrm{LiCuO}_{2}$ y $\mathrm{Li}_{2} \mathrm{CuO}_{2}$}

En la Tabla 7-1 se muestran los parámetros estructurales obtenidos de la relajación estructural de los sistemas $\mathrm{Li}_{2} \mathrm{CuO}_{2}$ y $\mathrm{LiCuO}_{2}$ utilizando el funcional PBE. Tal y como se aprecia en la tabla, el funcional PBE sobrestima, en ambos casos, los parámetros de celda. Con respecto a los ángulos, debido a que se utilizó la restricción de mantener la simetría de la celda unitaria, el único ángulo con la posibilidad de cambiar fue el ángulo $\beta$ del sistema $\mathrm{LiCuO}_{2}$, el cual se sobrestimó en $\sim 2.2 \%$. El impacto de esta sobrestimación de parámetros de red en el volumen es de $\sim 2.8 \%$ en el caso de $\mathrm{Li}_{2} \mathrm{CuO}_{2}$, mientras que para $\mathrm{LiCuO}_{2}$ es de $\sim 4.6 \%$, cabe mencionar que en este último, el porcentaje de sobrestimación fue mayor debido al cambio en el ángulo $\beta$. Al comparar estos resultados obtenidos mediante PBE, se puede notar que utilizando la aproximación $\mathrm{PBE}+\mathrm{U}$ se obtiene una menor sobrestima-

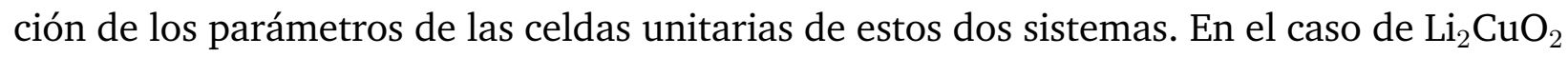
tanto PBE como PBE+U sobrestiman el parámetro a, mientras que en $\mathrm{LiCuO}_{2}$, c fue el más sobrestimado por PBE y $\mathrm{PBE}+\mathrm{U}$. 
Tabla 7-1: Parámetros estructurales de $\mathrm{Li}_{2} \mathrm{CuO}_{2}$ y $\mathrm{LiCuO}_{2}$ obtenidos con PBE y $\mathrm{PBE}+\mathrm{U}$

\begin{tabular}{ccccc}
\hline \multicolumn{5}{c}{$\mathrm{Li}_{2} \mathrm{CuO}_{2}$} \\
\hline \hline PBE & $a(\AA)$ & $b(\AA)$ & $c(\AA)$ & Volumen $(\AA)^{3}$ \\
PBE+U & 3.722 & 2.910 & 9.331 & 101.06 \\
Experimental $^{a}$ & 3.701 & 2.875 & 9.396 & 99.98 \\
Experimental $^{b}$ & $(3.660)$ & $(2.861)$ & $(9.389)$ & $(98.33)$ \\
\hline \hline & $(2.866)$ & $(9.397)$ & $(98.60)$ \\
\hline PBE & 5.889 & 2.739 & 5.846 & 78.77 \\
PBE+U & 5.836 & 2.713 & 5.737 & 76.89 \\
Experimental $^{c}$ & $(5.733)$ & $(2.718)$ & $(5.622)$ & $(75.33)$ \\
\hline \hline
\end{tabular}

a Parámetros experimentales de Ref [16]

b Parámetros experimentales de Ref [77]

c Parámetros experimentales de Ref [78]

\subsection{Datos estructurales de $\mathrm{Li}_{2} \mathrm{Cu}_{0.5} \mathrm{M}_{0.5} \mathrm{O}_{2}$ obtenidos con PBE+U}

\subsubsection{Parámetros estructurales}

Los resultados obtenidos con $\mathrm{PBE}+\mathrm{U}$ en los parámetros de celda para $\mathrm{Li}_{2} \mathrm{Cu}_{0.5} \mathrm{Co}_{0.5} \mathrm{O}_{2}$, $\mathrm{Li}_{2} \mathrm{Cu}_{0.5} \mathrm{Ni}_{0.5} \mathrm{O}_{2}$ y Li $\mathrm{Cu}_{0.5} \mathrm{Ti}_{0.5} \mathrm{O}_{2}$ son mostrados en la Tabla 7-2. A partir de esto se observa que el efecto de incluir un segundo metal de transición en la estructura cristalina de $\mathrm{Li}_{2} \mathrm{CuO}_{2}$ es diferente en cada parámetro.

En el caso del parámetro a, el efecto de Co y $\mathrm{Ni}$ es disminuirlo en $\sim 0.2 \%$ y $\sim 0.1 \%$, respectivamente, mientras que el efecto de Ti es aumentarlo en $\sim 0.8 \%$. En el caso de $\mathbf{b}$, el efecto Co, Ni y Ti es aumentarlo en $\sim 1 \%, \sim 0.5 \%$ y $\sim 3.6 \%$, respectivamente. Finalmente, para c, Co y Ti tienden a aumentarlo respecto a $\mathrm{Li}_{2} \mathrm{CuO}_{2}$, en $\sim 1 \%$ y $\sim 1.7 \%$, respectivamente; mientras que el efecto de $\mathrm{Ni}$ es disminuir este parámetro en $\sim 0.02 \%$. El impacto de esto en el volumen, en los tres casos, es aumentarlo en $\sim 1.9 \%, \sim 0.4 \%$ y $\sim 6.2 \%$ para el caso de $\mathrm{Li}_{2} \mathrm{Cu}_{0.5} \mathrm{Co}_{0.5} \mathrm{O}_{2}, \mathrm{Li}_{2} \mathrm{Cu}_{0.5} \mathrm{Ni}_{0.5} \mathrm{O}_{2}$ y Li $\mathrm{Cu}_{0.5} \mathrm{Ti}_{0.5} \mathrm{O}_{2}$, respectivamente. 
Tabla 7-2: Parámetros estructurales calculados con PBE+U de $\mathrm{Li}_{2} \mathrm{Cu}_{0.5} \mathrm{Co}_{0.5} \mathrm{O}_{2}$, $\mathrm{Li}_{2} \mathrm{Cu}_{0.5} \mathrm{Ni}_{0.5} \mathrm{O}_{2}$ y $\mathrm{Li}_{2} \mathrm{Cu}_{0.5} \mathrm{Ti}_{0.5} \mathrm{O}_{2}$, comparados con $\mathrm{Li}_{2} \mathrm{CuO}_{2}$. Los parámetros experimentales disponibles son mostrados entre paréntesis.

\begin{tabular}{|c|c|c|c|c|}
\hline Sistema & $a(\AA)$ & $b(\AA)$ & $c(\AA)$ & Volumen $\left(\AA^{3}\right)$ \\
\hline $\mathrm{Li}_{2} \mathrm{CuO}_{2}$ & 3.701 & 2.875 & 9.396 & 99.977 \\
\hline $\mathbf{L i}_{2} \mathbf{C u}_{0.5} \mathbf{C o}_{0.5} \mathbf{O}_{2}$ & 3.694 & 2.906 & 9.490 & 101.873 \\
\hline $\mathbf{L i}_{2} \mathbf{C u}_{0.5} \mathbf{N i}_{0.5} \mathbf{O}_{2}$ & 3.696 & 2.890 & 9.394 & 100.341 \\
\hline $\mathbf{L i}_{2} \mathbf{C u}_{0.5} \mathbf{N i}_{0.5} \mathbf{O}_{2}{ }^{\mathbf{a}}$ & (3.692) & (2.827) & (9.238) & (96.42) \\
\hline $\mathbf{L i}_{2} \mathbf{C u}_{0.5} \mathbf{T i}_{0.5} \mathbf{O}_{2}$ & 3.731 & 2.979 & 9.555 & 106.200 \\
\hline
\end{tabular}

a Parámetros experimentales de Ref [16]

Resulta importante mencionar que, para la solución sólida $\mathrm{Li}_{2} \mathrm{Cu}_{0.5} \mathrm{Ni}_{0.5} \mathrm{O}_{2}$, existen datos estructurales reportados y a partir de éstos se puede concluir que con la estructura cristalina que se propuso en este trabajo para este sistema, $\mathrm{PBE}+\mathrm{U}$ sobrestima los parámetros estructurales a, b y c en $\sim 0.1 \%, \sim 2.2 \%$ y $\sim 1.7 \%$ respectivamente, teniendo un impacto en un aumento del volumen en $\sim 4.1 \%$ respecto al dato experimental. Por otro lado, al comparar los datos experimentales de a, b y c entre $\mathrm{Li}_{2} \mathrm{CuO}_{2}$ y $\mathrm{Li}_{2} \mathrm{Cu}_{0.5} \mathrm{Ni}_{0.5} \mathrm{O}_{2}$, notamos que el efecto de insertar $\mathrm{Ni}$ en la estructura cristalina $\mathrm{de}_{\mathrm{Li}_{2}} \mathrm{CuO}_{2}$, es aumentar a y disminuir $\mathbf{b}$ y $\mathbf{c}$. Esta tendencia no es obtenida con los datos de este trabajo ya que a y $\mathbf{c}$ tienden a disminuir y $\mathbf{b}$ aumenta, por efecto de los átomos de Ni.

Sin embargo, es necesario aclarar que hasta el momento no existen datos experimentales suficientes para la construcción de la estructura cristalina de este sistema, por lo que la estructura propuesta en este trabajo para la solución sólida $\mathrm{Li}_{2} \mathrm{Cu}_{0.5} \mathrm{Ni}_{0.5} \mathrm{O}_{2}$, es la más simple $^{\mathrm{a}}$, (alternando láminas de $\mathrm{Cu}-\mathrm{O}$ y Ni-O sobre c), por lo que existen otras posibilidades de representar la misma relación $\mathrm{Cu}$ :Ni que pueden impactar tanto en aspectos estructurales como en la estructura electrónica.

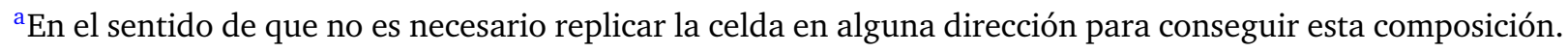




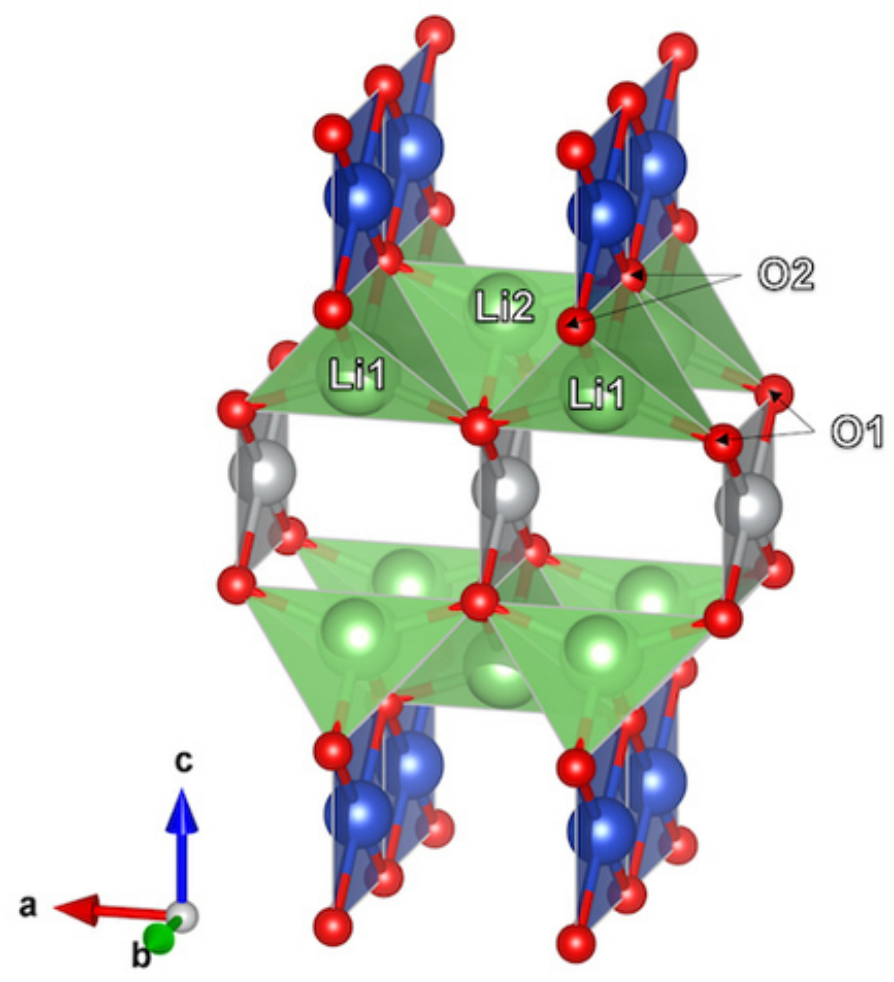

Figura 7-1: Estructura general para los sistemas $\mathrm{Li}_{2} \mathrm{Cu}_{0.5} \mathrm{M}_{0.5} \mathrm{O}_{2}$. Las esferas azules representan átomos de $\mathrm{Cu}$, verdes a $\mathrm{Li}$, rojas a $\mathrm{O}$ y gris para representar a los átomos $\mathrm{M}=\mathrm{Cu}$, Co, $\mathrm{Ni}$ o Ti. Los diferentes tipos de átomos de $\mathrm{O}$ y Li son etiquetados con 1 y 2 .

Para el análisis de los resultados de esta subsección es necesario ver la Figura 7-1. En ella se aprecia la presencia de dos tipos de átomos de O (O1 y O2) y de Li (Li1 y Li2). Los O1 corresponden a átomos de $\mathrm{O}$ que conforman la coordinación plana cuadrada de $\mathrm{MO}_{4}(\mathrm{M}$ $=\mathrm{Co}$, Ni o Ti, de color gris en la Figura 7-1); mientras que los $\mathrm{O} 2$ conforman los planos cuadrados de $\mathrm{CuO}_{4}$ (de color azul en la Figura 7-1). Por otra parte, los Li1 están unidos a dos $\mathrm{O} 2$ del mismo plano cuadrado $\mathrm{CuO}_{4}$ y dos $\mathrm{O} 1$ de planos cuadrados $\mathrm{MO}_{4}$ distintos; mientras que los Li2 están unidos a dos $\mathrm{O} 2$ de diferentes planos cuadrados $\mathrm{CuO}_{4}$ y dos $\mathrm{O} 1$ del mismo plano cuadrado $\mathrm{MO}_{4}$.

Las distancias de enlace Cu-O, M-O y Li-O son mostradas en la Tabla 7-3. En ella se puede apreciar que el mayor cambio respecto a $\mathrm{Li}_{2} \mathrm{CuO}_{2}$, ocurre en la solución sólida $\mathrm{Li}_{2} \mathrm{Cu}_{0.5} \mathrm{Ti}_{0.5} \mathrm{O}_{2}$, en el cual, con la excepción de las distancias Li1-O2 y Li2-O2, todas las demás distancias son mayores respecto a las de $\mathrm{Li}_{2} \mathrm{CuO}_{2}$. Resulta interesante señalar que 
las distancias Li1-O2 y Li2-O2 son más pequeñas, por lo que se puede inferir que el efecto de incluir Ti en la estructura cristalina de $\mathrm{Li}_{2} \mathrm{CuO}_{2}$, es que las láminas de $\mathrm{TiO}_{4}$ comprimen a las láminas de $\mathrm{CuO}_{4}$ ocasionando que los átomos de litio (Li1 y Li2) se aproximen más a los átomos O2. Por otro lado, el sistema con el menor cambio en las distancias de enlace respecto a $\mathrm{Li}_{2} \mathrm{CuO}_{2}$, fue $\mathrm{Li}_{2} \mathrm{Cu}_{0.5} \mathrm{Ni}_{0.5} \mathrm{O}_{2}$, en el cual, cuatro distancias se mantuvieron sin cambios (Ni-O1, Cu-O2, Li1-O2, Li2O1) y las demás fueron ligeramente mayores. Por último, el efecto de incluir Co no modifica las distancias Cu-O2 y Li1-O2 y las demás son más grandes en $0.01 \AA$.

Tabla 7-3: Distancias de enlace obtenidas con $\mathrm{PBE}+\mathrm{U}$ para las soluciones sólidas $\mathrm{Li}_{2} \mathrm{Cu}_{0.5} \mathrm{Ni}_{0.5} \mathrm{O}_{2}, \mathrm{Li}_{2} \mathrm{Cu}_{0.5} \mathrm{Co}_{0.5} \mathrm{O}_{2}$ y Li $\mathrm{Cu}_{0.5} \mathrm{Ti}_{0.5} \mathrm{O}_{2}$, comparadas con $\mathrm{Li}_{2} \mathrm{CuO}_{2}$. Los datos experimentales disponibles son mostrados entre paréntesis.

\begin{tabular}{ccccc}
\hline \hline Distancia, $d(\AA)$ & \multicolumn{4}{c}{ Sistemas } \\
\hline M-O1 & $\mathbf{L i}_{2} \mathbf{C u O}_{2}$ & $\mathbf{L i}_{2} \mathbf{C u}_{0.5} \mathbf{C o}_{0.5} \mathbf{O}_{2}$ & $\mathbf{L i}_{2} \mathbf{C u}_{0.5} \mathbf{N i}_{0.5} \mathbf{O}_{2}$ & $\mathbf{L i}_{2} \mathbf{C u}_{0.5} \mathbf{T i}_{0.5} \mathbf{O}_{2}$ \\
\hline & 1.97 & 2.0 & 1.97 & 2.01 \\
Cu-O2 & $(1.96)^{\mathrm{a}}$ & - & $(1.92)^{\mathrm{b}}$ & - \\
& 1.97 & 1.97 & 1.97 & 2.05 \\
Li1-O1 & $(1.96)^{\mathrm{a}}$ & - & $(1.92)^{\mathrm{b}}$ & - \\
& 1.96 & 1.97 & 1.97 & 2.03 \\
Li1-O2 & $(1.95)^{\mathrm{a}}$ & - & $(1.95)^{\mathrm{b}}$ & - \\
& 1.98 & 1.98 & 1.98 & 1.94 \\
Li2-O1 & $(1.98)^{\mathrm{a}}$ & - & $(1.98)^{\mathrm{b}}$ & - \\
& 1.98 & 1.99 & 1.98 & 2.18 \\
Li2-O2 & $(1.98)^{\mathrm{a}}$ & - & $(1.98)^{\mathrm{b}}$ & - \\
& 1.96 & 1.97 & 1.97 & 1.91 \\
& $(1.95)^{\mathrm{a}}$ & - & $(1.95)^{\mathrm{b}}$ & - \\
\hline \hline
\end{tabular}

a Parámetros experimentales de Ref [77]

b Parámetros experimentales de Ref [16] 


\subsection{Estado de magnetización obtenido para cada sistema con el fun- cional HSE06}

De acuerdo a la fórmula unidad de $\mathrm{Li}_{2} \mathrm{CuO}_{2}$ y $\mathrm{LiCuO}_{2}$, considerando que los oxígenos de las redes cristalinas están como $\mathrm{O}^{2-}$ y los litios como $\mathrm{Li}^{1+}$ implica que los átomos de cobre se encuentran en estado de oxidación ${ }^{2+b}$ (en el caso de $\mathrm{Li}_{2} \mathrm{CuO}_{2}$ ), mientras que en el caso de $\mathrm{LiCuO}_{2}$ se encuentran como $\mathrm{Cu}^{3+}$. En la Tabla 7-4 se muestran las poblaciones electrónicas en los orbitales $d^{\mathrm{c}}$ y las magnetizaciones atómicas ${ }^{\mathrm{d}}$ de cada uno de los metales de transición presentes en los sistemas estudiados. Con esta información es posible hacer una propuesta del estado de oxidación de cada uno de ellos, así como el acoplamiento entre ellos de sus estados de espín.

Tabla 7-4: Poblaciones electrónicas en los orbitales $d$, magnetización total y atómica para cada metal de transición, obtenidas con HSE06

\begin{tabular}{clccccc}
\hline Sistema & \multicolumn{2}{l}{ Población electrónica en orbitales } & \multicolumn{3}{c}{ Magnetización } \\
& $\mathbf{C u}$ & $\mathbf{M}(\mathbf{M}=\mathbf{C o}, \mathbf{N i} \mathbf{o} \mathbf{~ T i})$ & $\mathbf{C u}$ & $\mathbf{M}$ & Total \\
\hline $\mathbf{L i C u O} \mathbf{O}_{2}$ & 9.0 & - & 0 & - & 0 \\
$\mathbf{L i}_{2} \mathbf{C u O}_{2}$ & 9.1 & - & \pm 0.66 & - & 0 \\
$\mathbf{L i}_{2} \mathbf{C u}_{0.5} \mathbf{C o}_{0.5} \mathbf{O}_{2}$ & 9.1 & 7.0 & -0.66 & 2.70 & 2.0 \\
$\mathbf{L i}_{2} \mathbf{C u}_{0.5} \mathbf{N i}_{0.5} \mathbf{O}_{2}$ & 9.1 & 8.1 & -0.66 & 1.69 & 1.0 \\
$\mathbf{L i}_{2} \mathbf{C u}_{0.5} \mathbf{T i}_{0.5} \mathbf{O}_{2}$ & 9.2 & 1.7 & -0.49 & -0.42 & -1.0 \\
\hline \hline
\end{tabular}

Dentro de la Teoría del Campo Cristalino (TCC) [99], una coordinación plana cuadrada desdobla a los orbitales $d$ de acuerdo a la Figura 7-2 [100]. En todos los sistemas, las poblaciones electrónicas en los orbitales $d$ de los átomos de $\mathrm{Cu}$, corresponden con una configuración electrónica tipo $3 d^{9}$ y es posible proponer que el estado de oxidación de $\mathrm{Cu}$ en todos los sistemas, es $2+$. Sin embargo, también es necesario considerar la magnetización sobre cada átomo, en donde con excepción de $\mathrm{LiCuO}_{2}{ }^{\mathrm{e}}$, todos los sistemas muestran una magnetización correspondiente a una configuración electrónica tipo $3 d^{9}$ y por lo tanto un estado de oxidación $2+$.

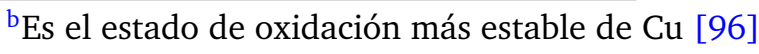

'Son proyecciones de orbitales $d$ dentro de cada EA.

${ }^{\mathrm{d}}$ Obtenidas a partir de un análisis de población de Bader $[97,98]$

eEn el caso de $\mathrm{LiCuO}_{2}$, la magnetización sobre $\mathrm{Cu}$ de 0 es correspondiente a una configuración electrónica tipo $3 d^{8}$ y por ello un estado de oxidación $3+$
} 


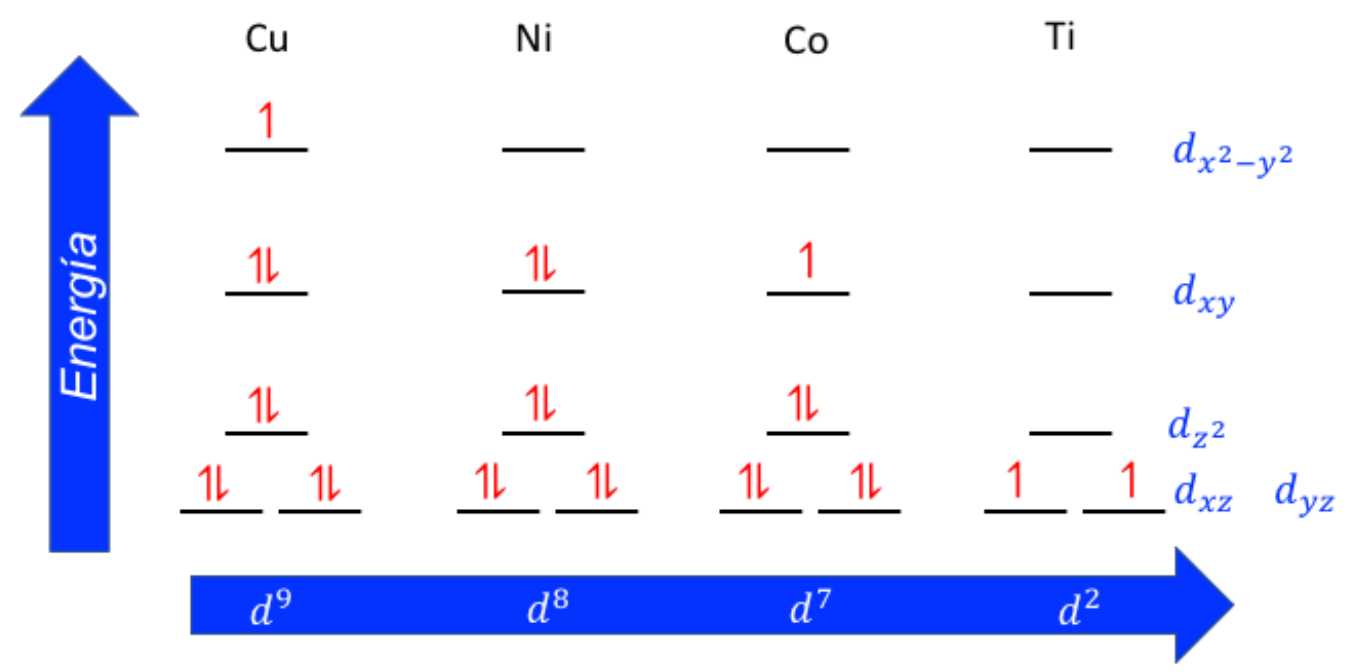

Figura 7-2: Llenado de orbitales $d$ para configuraciones electrónicas en bajo espín $\mathrm{d}^{9}, \mathrm{~d}^{8}$, $\mathrm{d}^{7} \mathrm{y} \mathrm{d}^{2}$ que corresponderían a los átomos de $\mathrm{Cu}, \mathrm{Ni}$, Co y Ti, respectivamente, en estado de oxidación $2+$.

Las poblaciones electrónicas en los orbitales $d$ de los átomos de Ni, Co y Ti, corresponden con las configuraciones electrónicas tipo $3 d^{8}, 3 d^{7}$ y $3 d^{2}$, respectivamente; las cuales, permiten proponer que los estados de oxidación para los tres átomos es $2+^{\mathrm{f}}$. Si se asume que estas configuraciones se ordenan en un esquema de bajo espín ${ }^{g}$, el diagrama queda como el mostrado en la Figura 7-2.

En el caso de $\mathrm{Li}_{2} \mathrm{CuO}_{2}$, el valor de magnetización de cada uno de los dos átomos de $\mathrm{Cu}$ de la celda unitaria, indican que se orientan de forma tal que la magnetización total es cero (Tabla 7-4) y presenta un ordenamiento antiferromagnético, tal y como se ha propuesto experimentalmente [102].

Respecto a los sistemas $\mathrm{Li}_{2} \mathrm{Cu}_{0.5} \mathrm{M}_{0.5} \mathrm{O}_{2}$, las magnetizaciones obtenidas no son congruentes con el desdoblamiento de los orbitales $d$ esperado por TCC. En el caso de $\mathrm{Li}_{2} \mathrm{Cu}_{0.5} \mathrm{Ni}_{0.5} \mathrm{O}_{2}$, la magnetización obtenida sobre $\mathrm{Ni}$ corresponde a tener dos electrones desapareados, esta configuración electrónica se acopla con la del átomo de $\mathrm{Cu}$ en forma antiferromagnética. Una observación similar puede hacerse en el sistema $\mathrm{Li}_{2} \mathrm{Cu}_{0.5} \mathrm{Co}_{0.5} \mathrm{O}_{2}$, en el cual la magnetización atómica sobre Co, de $\sim 3$, también presenta un acoplamiento antiferromagnético entre el electrón desapareado de $\mathrm{Cu}$ y los tres de Co. Sin embargo, en el caso

\footnotetext{
${ }^{\mathrm{f}}$ Este estado de oxidación es el más estable para Ni y Co, mientras que en el caso de Ti el más estable es $4+[96]$

${ }^{g}$ Es decir, existe el menor número de electrones desapareados en la configuración electrónica [101].
} 
$\mathrm{Li}_{2} \mathrm{Cu}_{0.5} \mathrm{Ti}_{0.5} \mathrm{O}_{2}$, las magnetizaciones atómicas del $\mathrm{Ti}$ y $\mathrm{Cu}$, muestran un acoplamiento ferromagnético.

\subsection{Análisis de la densidad de estados proyectada}

En la Figura 7-3 se muestran las DOS- $p$ de $\mathrm{Li}_{2} \mathrm{CuO}_{2}$ (Figura 7-3-a) y los sistemas $\mathrm{Li}_{2} \mathrm{Cu}_{0.5} \mathrm{M}_{0.5} \mathrm{O}_{2}$ (Figura 7-3-b a -d). En cada una de estas gráficas se resalta una región (región de interés) delimitada por el nivel de Fermi (línea punteada de color negro) y un valor de energía tal que la integral de la DOS cumple la condición de la ecuación 6-7(línea punteada de color violeta).

Tal y como se aprecia en el panel a) de la (Figura 7-3), los estados comprendidos dentro de la región de interés, corresponden en su mayoría a $\mathrm{O}$ con un menor contenido de los pertenecientes a $\mathrm{Cu}$ tal como se ha reportado previamente [24]. Esta proporción se invierte al observar la región de estados desocupados de 2 a $2.5 \mathrm{eV}$ mas allá del nivel de Fermi (hacia la derecha). Además, se observa un gap de $\sim 1.8 \mathrm{eV}$.

Cuando la estructura cristalina de $\mathrm{Li}_{2} \mathrm{CuO}_{2}$ es sustituida en $50 \%$ de los átomos de $\mathrm{Cu}$ por Co $\left(\mathrm{Li}_{2} \mathrm{Cu}_{0.5} \mathrm{Co}_{0.5} \mathrm{O}_{2}\right.$, panel b) de la Figura 7-3), la contribución de los estados de $\mathrm{O}$ disminuye pero no dejan de ser mayoritarios en la región de pérdida de un electrón. En segundo lugar aparecen los estados correspondientes a Co y en tercero los de $\mathrm{Cu}$. Al revisar la región 1.5 a $3 \mathrm{eV}$ de la banda de conducción, al igual que $\mathrm{Li}_{2} \mathrm{CuO}_{2}$, los primeros estados que pudieran ser ocupados, corresponden en su mayoría a $\mathrm{Cu}$ y en segundo lugar a $\mathrm{O}$; a valores más altos de energía, los estados de Co son los dominantes; además el gap es de $\sim 1.5 \mathrm{eV}$. El efecto de sustitución de $\mathrm{Ni}\left(\mathrm{Li}_{2} \mathrm{Cu}_{0.5} \mathrm{Ni}_{0.5} \mathrm{O}_{2}\right.$, panel c) de la Figura 7-3)es muy parecido al del Co, tanto en la región de retiro de un electrón, como en la región de estados desocupados en un intervalo de 1.5 a $3 \mathrm{eV}$ por arriba del nivel de Fermi y el gap es de $\sim 1.7 \mathrm{eV}$.

Sin embargo, cuando los átomos de Ti sustituyen a $\mathrm{Cu}$, el comportamiento de la DOS-p es diferente $\left(\mathrm{Li}_{2} \mathrm{Cu}_{0.5} \mathrm{Ti}_{0.5} \mathrm{O}_{2}\right.$, panel d) de la Figura 7-3). El sistema no muestra gap, y el mayor contribuyente en la región de retiro de un electrón corresponde a los estados del $\mathrm{Cu}$, seguidos por los correspondientes a Ti y finalmente por aquellos de $\mathrm{O}$. La región de estados desocupados, hasta $2.5 \mathrm{eV}$ más allá del nivel de Fermi, corresponde en su mayoría a estados propios del $\mathrm{Ti}$, seguidos por los de $\mathrm{O}$ y en tercer lugar los de $\mathrm{Cu}$. Podemos resumir que en orden decreciente, el mayor efecto en la disminución de los estados de oxígeno en la región de interés fue la sustitución con $\mathrm{Ti}>\mathrm{Co}>\mathrm{Ni}$, por otro lado en ese orden aumenta el gap, $\mathrm{Ti}<\mathrm{Co}<\mathrm{Ni}$. 

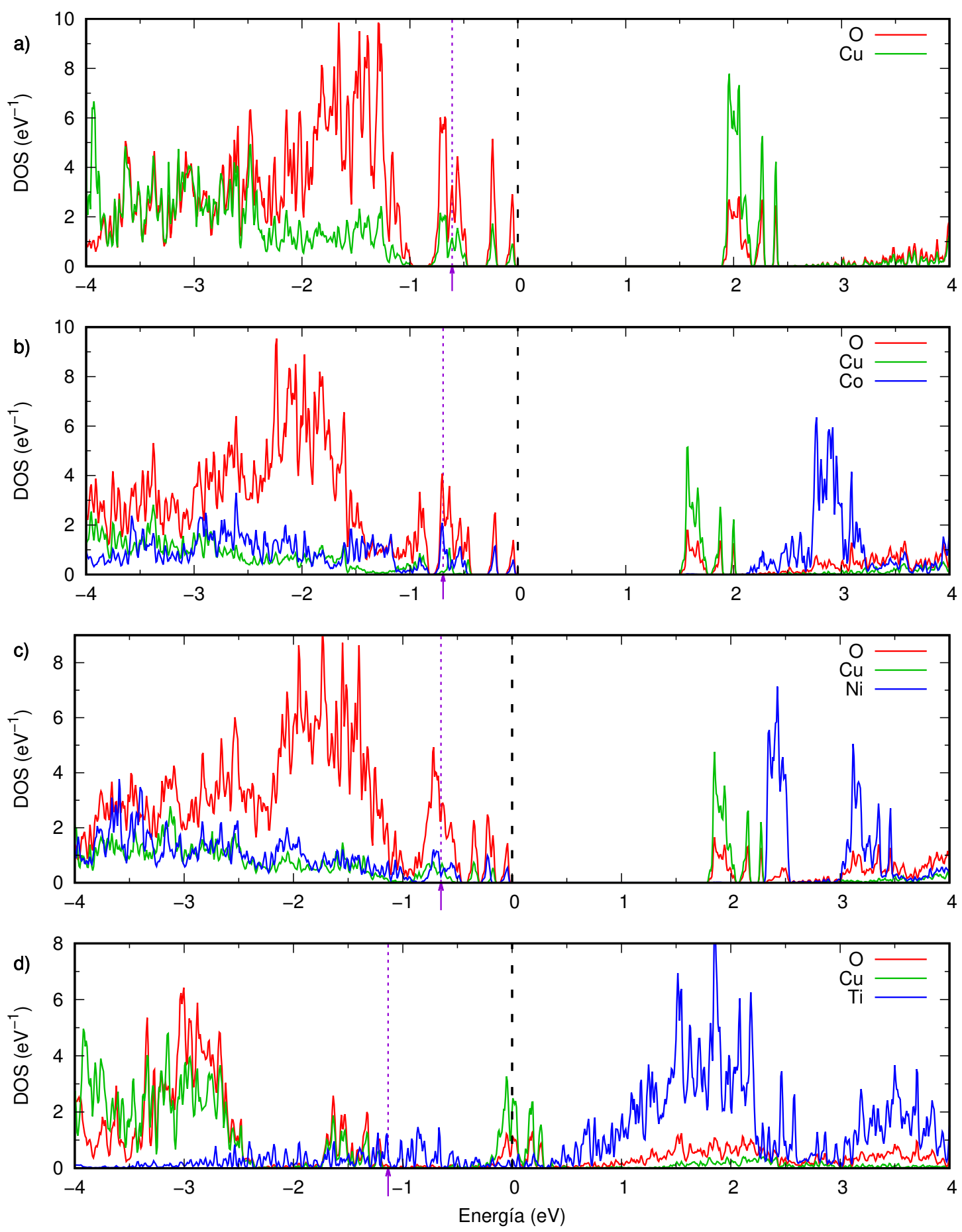

Figura 7-3: $D O S-p$ de a) $\mathrm{Li}_{2} \mathrm{CuO}_{2}$, b) $\mathrm{Li}_{2} \mathrm{Cu}_{0.5} \mathrm{Co}_{0.5} \mathrm{O}_{2}$, c) $\mathrm{Li}_{2} \mathrm{Cu}_{0.5} \mathrm{Ni}_{0.5} \mathrm{O}_{2}$ y d) $\mathrm{Li}_{2} \mathrm{Cu}_{0.5} \mathrm{Ti}_{0.5} \mathrm{O}_{2}$, obtenidas con $\mathrm{PBE}+\mathrm{U}$. La línea punteada de color negro indica el nivel de Fermi de cada sistema. El nivel de Fermi y la línea punteada de color violeta encierran el intervalo de energía donde la DOS integra a 1 electrón. 
Como se ha mencionado antes, el proceso redox de $\mathrm{Li}_{2} \mathrm{CuO}_{2}$ comprende los átomos de metal de transición así como los átomos de oxígeno, es decir los planos cuadrados $\mathrm{Cu}-\mathrm{O}$. Por lo que se graficó la DOS-p proyectada por ambiente químico M-O1 ( $\mathrm{M}=\mathrm{Co}-$, Ni- o Tioxígeno tipo 1) y Cu-O2 (cobre-oxígeno tipo 2), Figura 7-1, con el propósito de conocer el plano cuadrado más propenso a oxidarse.
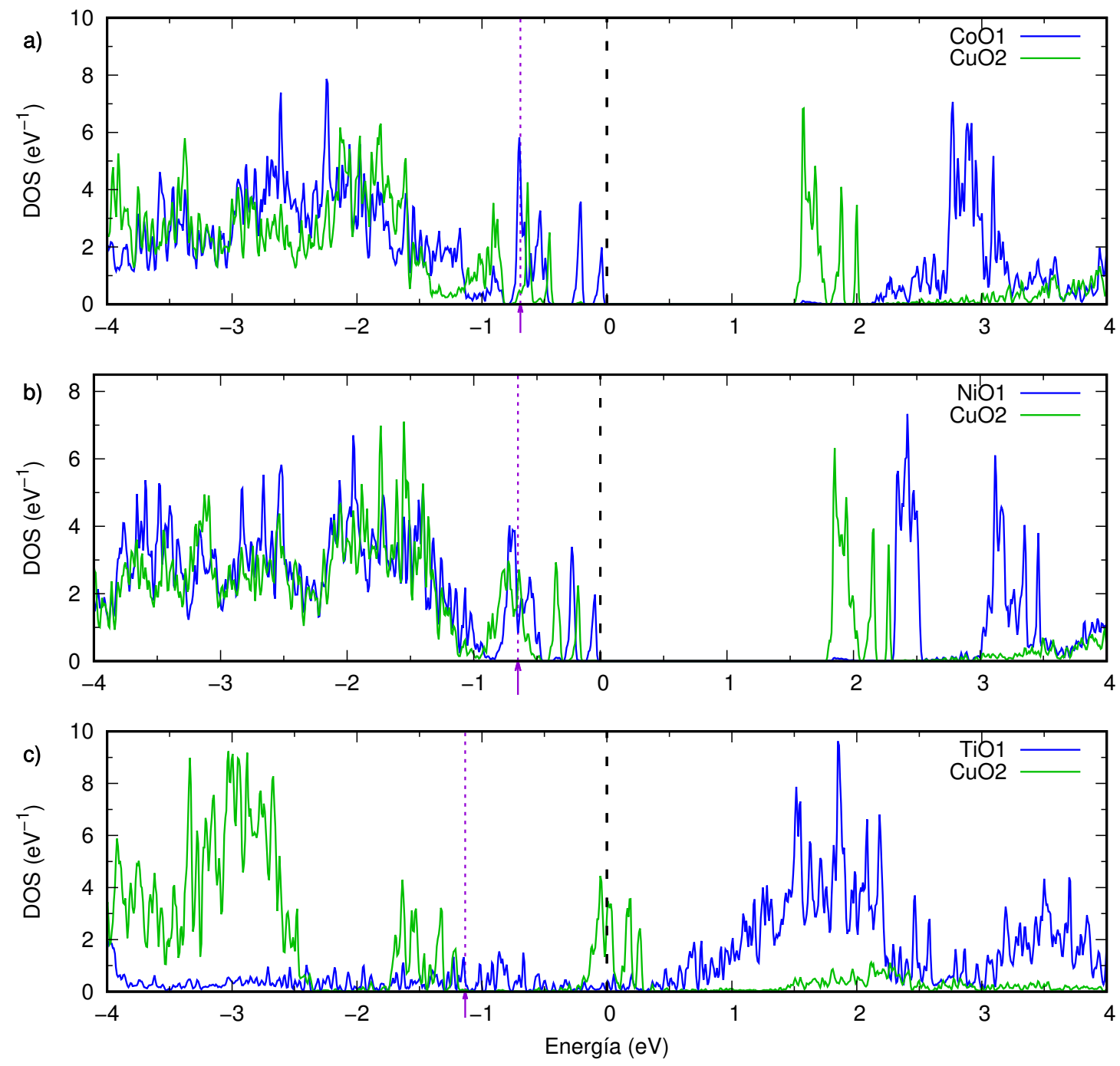

Figura 7-4: $D O S$ - $p$ por ambiente químico Cu-O2 y M-O1 (ver Figura 7-1), de los sistemas a) $\mathrm{Li}_{2} \mathrm{Cu}_{0.5} \mathrm{Co}_{0.5} \mathrm{O}_{2}$, b) $\mathrm{Li}_{2} \mathrm{Cu}_{0.5} \mathrm{Ni}_{0.5} \mathrm{O}_{2}$ y c) $\mathrm{Li}_{2} \mathrm{Cu}_{0.5} \mathrm{Ti}_{0.5} \mathrm{O}_{2}$, obtenidas con PBE+ $\mathrm{U}$. La línea punteada de color negro indica el nivel de Fermi de cada sistema. El nivel de Fermi y la línea punteada de color violeta encierran el intervalo de energía donde la DOS integra a 1 electrón.

Tal y como se aprecia en la Figura 7-4, la región de interés en $\mathrm{Li}_{2} \mathrm{Cu}_{0.5} \mathrm{Co}_{0.5} \mathrm{O}_{2}$ donde se puede perder un electrón está compartida entre los planos cuadrados Cu-O2 y Co-O1 sien- 
do ligeramente mayoritario este último. Los primeros estados desocupados corresponden a estados de Cu-O2 seguidos por aquellos de Co-O1. Con respecto al $\mathrm{Li}_{2} \mathrm{Cu}_{0.5} \mathrm{Ni}_{0.5} \mathrm{O}_{2}$, el efecto es muy similar al observado en el caso anterior, existe una competencia entre los planos cuadrados $\mathrm{Cu}-\mathrm{O} 2$ y Co-O1. También en este sistema los primeros estados desocupados corresponden a estados de los cuadrados $\mathrm{Cu}-\mathrm{O} 2$ seguidos por aquellos de $\mathrm{Co}-\mathrm{O} 1$. Finalmente, en el caso de $\mathrm{Li}_{2} \mathrm{Cu}_{0.5} \mathrm{Ti}_{0.5} \mathrm{O}_{2}$, los estados correspondientes al plano cuadrado Ti-O1 resultan ser los más susceptibles a oxidarse. Cabe mencionar que en el caso de $\mathrm{Li}_{2} \mathrm{Cu}_{0.5} \mathrm{Co}_{0.5} \mathrm{O}_{2}$ y $\mathrm{Li}_{2} \mathrm{Cu}_{0.5} \mathrm{Ni}_{0.5} \mathrm{O}_{2}$ el proceso redox va más allá del metal de transición, abarca a los oxígenos coordinados a éste (tipo 1 o 2). En el caso de $\mathrm{Li}_{2} \mathrm{Cu}_{0.5} \mathrm{Ti}_{0.5} \mathrm{O}_{2}$, los estados del ambiente químico Ti-O1 son los que contribuyen más aunque en realidad, de este ambiente químico, la contribución de estados por parte de Ti son los mayoritarios. En la Figura 7-5 se muestran las DOS-p obtenidas con el funcional HSE06. En el caso de $\mathrm{Li}_{2} \mathrm{CuO}_{2}$, tal y como se ha descrito antes [24], los estados correspondientes a los átomos de oxígeno son los mayoritarios en la región de pérdida de un electrón, mientras que los primeros estados vacíos son en su mayoría pertenecientes a $\mathrm{Cu}$ y muestra un gap de $\sim 3.2$ eV. En el sistema $\mathrm{Li}_{2} \mathrm{Cu}_{0.5} \mathrm{Co}_{0.5} \mathrm{O}_{2}$, sin embargo, existe una competencia entre los estados correspondientes a Co y los de $\mathrm{O}$, quedando en tercer lugar los estados de $\mathrm{Cu}$. Nuevamente los primeros estados desocupados comprenden en primer lugar a los de $\mathrm{Cu}$ y presenta un gap de $\sim 2.6 \mathrm{eV}$. Este mismo comportamiento, pero en menor proporción, es observado en $\mathrm{Li}_{2} \mathrm{Cu}_{0.5} \mathrm{Ni}_{0.5} \mathrm{O}_{2}$, aquí los estados ocupados de $\mathrm{O}$ son mayoritarios y nuevamente los estados correspondientes a $\mathrm{Cu}$ quedan en tercer lugar. Sin embargo, los estados desocupados más próximos al nivel de Fermi pertenecen a Ni y el valor del gap es de $\sim 2.5 \mathrm{eV}$. Por último, el sistema $\mathrm{Li}_{2} \mathrm{Cu}_{0.5} \mathrm{Ti}_{0.5} \mathrm{O}_{2}$ muestra que la sustracción de un electrón es altamente probable que se presente en los estados pertenecientes a Ti. A diferencia de los sistemas anteriores, este es el único caso dónde se observa ausencia de un gap y un valor pequeño para la densidad de estados en el nivel de Fermi. También se observa que los estados desocupados pertenecen en su mayoría a Ti. Nuevamente, al igual que los resultados obtenidos con $\mathrm{PBE}+\mathrm{U}$, el orden en disminución de estados de oxígeno en la región de interés es $\mathrm{Ti}>\mathrm{Co}$ $>\mathrm{Ni}$ y en ese orden (pero ascendente) es la comparación resultante entre los gap de estos sistemas. 

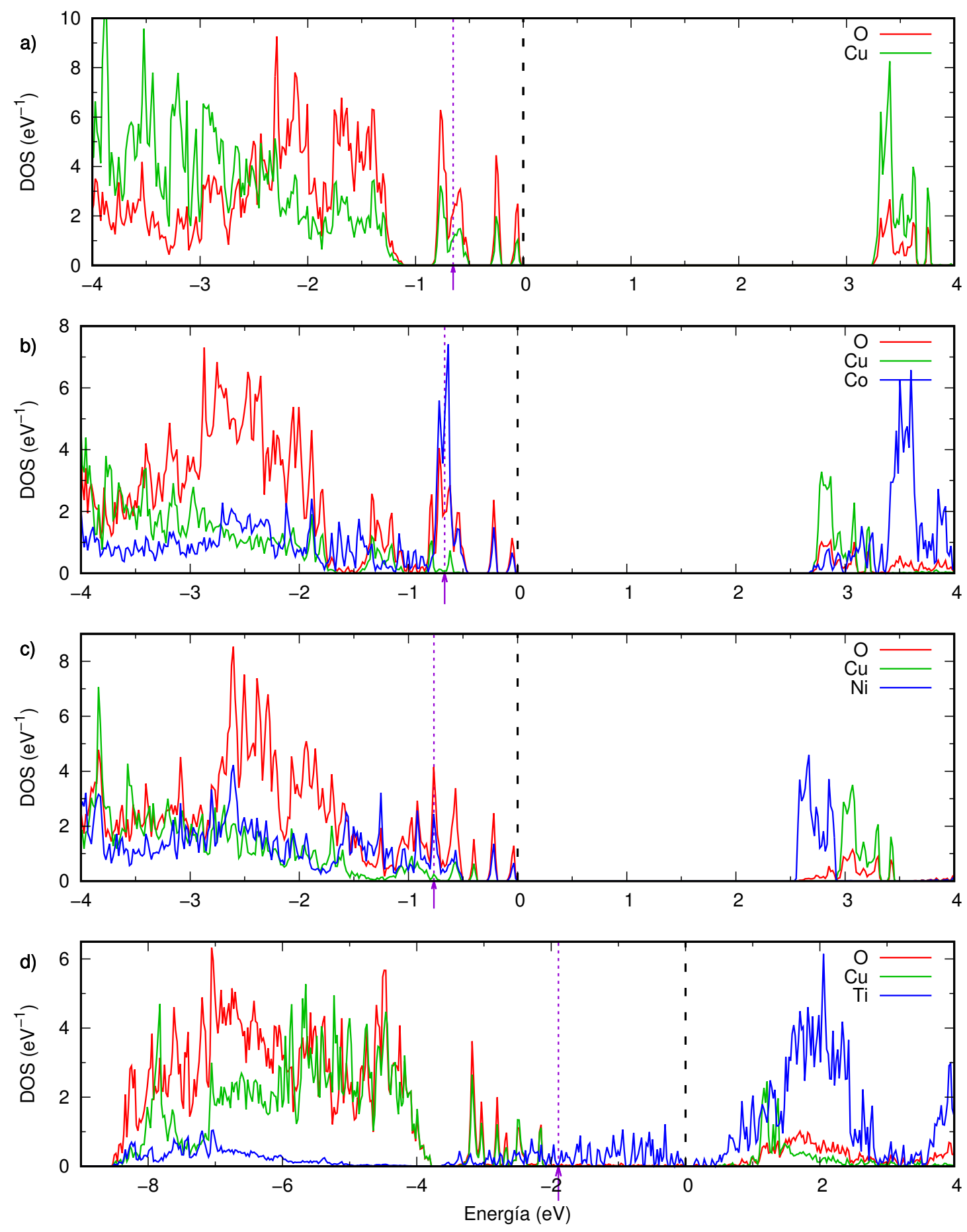

Figura 7-5: $D O S$ - $p$ de a) $\mathrm{Li}_{2} \mathrm{CuO}_{2}$, b) $\mathrm{Li}_{2} \mathrm{Cu}_{0.5} \mathrm{Co}_{0.5} \mathrm{O}_{2}$, c) $\mathrm{Li}_{2} \mathrm{Cu}_{0.5} \mathrm{Ni}_{0.5} \mathrm{O}_{2}$ y d) $\mathrm{Li}_{2} \mathrm{Cu}_{0.5} \mathrm{Ti}_{0.5} \mathrm{O}_{2}$, obtenidas con HSE06. La línea punteada de color negro indica el nivel de Fermi en cada sistema. El nivel de Fermi y la línea punteada de color violeta encierran el intervalo de energía donde la DOS integra a 1 electrón. 
Análogo a la Figura 7-4, en la Figura 7-6 se muestra la $D O S-p$ por ambiente químico, CuO2 y M-O1 ( $\mathrm{M}=\mathrm{Co}$, Ni o Ti), obtenida con el funcional HSE06, para los sistemas $\mathrm{Li}_{2} \mathrm{CuO}_{2}$ y $\mathrm{Li}_{2} \mathrm{Cu}_{0.5} \mathrm{M}_{0.5} \mathrm{O}_{2}$. Cuando la composición de la estructura cristalina de $\mathrm{Li}_{2} \mathrm{CuO}_{2}$ es modificada a $\mathrm{Li}_{2} \mathrm{Cu}_{0.5} \mathrm{Co}_{0.5} \mathrm{O}_{2}$, se obtiene que los estados con mayor disponibilidad a ser oxidados (es decir, aquéllos que se ven afectados por la sustracción de un electrón), corresponden a los pertenecientes a los planos cuadrados de Co-O1. En el caso de $\mathrm{Li}_{2} \mathrm{Cu}_{0.5} \mathrm{Ni}_{0.5} \mathrm{O}_{2}$ se aprecia el mismo comportamiento pero en menor magnitud con los planos cuadrados de Ni-O1. Por último, en el caso de $\mathrm{Li}_{2} \mathrm{Cu}_{0.5} \mathrm{Ti}_{0.5} \mathrm{O}_{2}$, también se obtiene un sistema sin gap y los planos cuadrados de Ti-O1 son los únicos que aparecen en la región de interés, aunque al igual que lo obtenido con $\mathrm{PBE}+\mathrm{U}$, los estados correspondientes a Ti son los dominantes. 

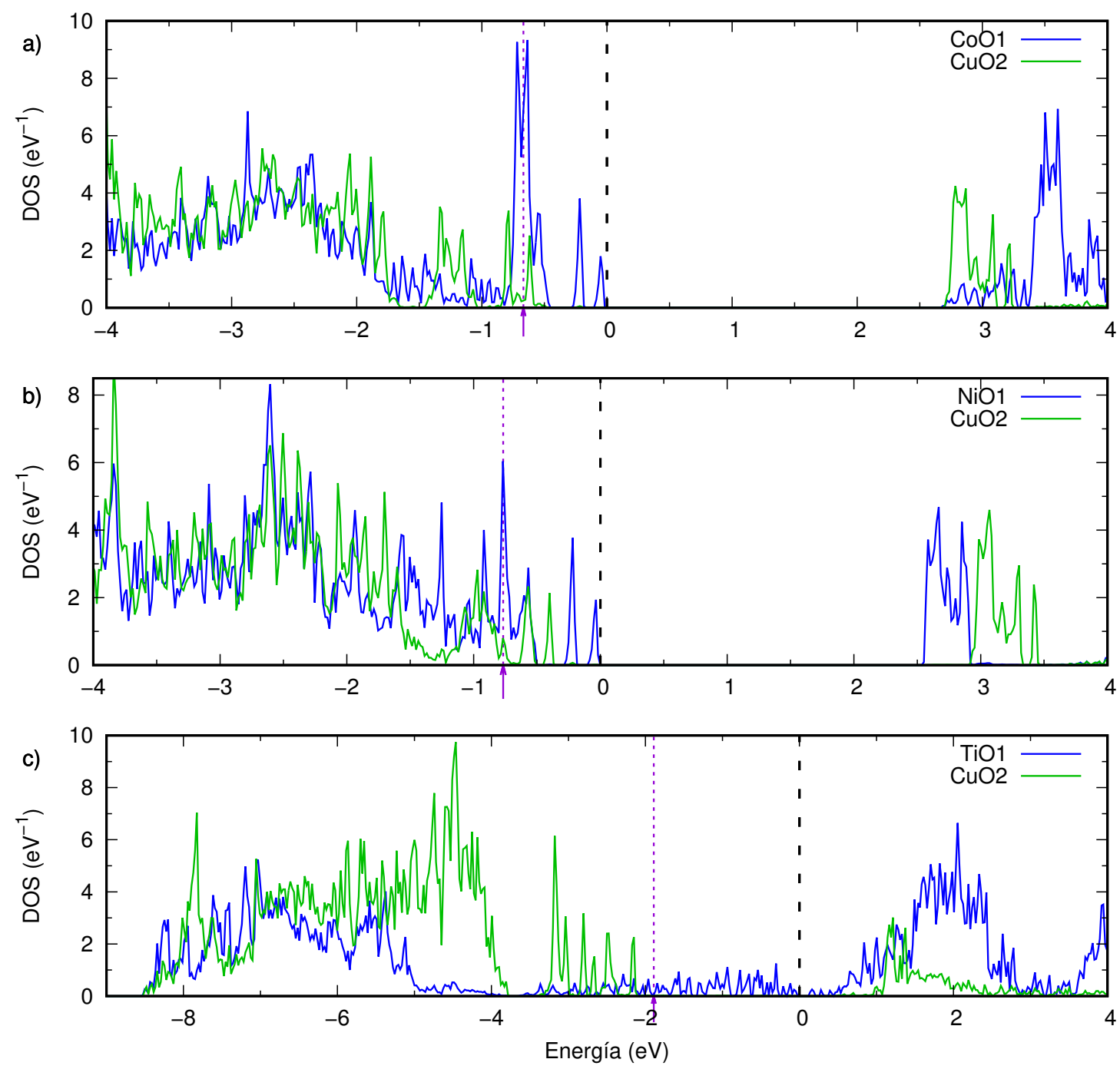

Figura 7-6: $D O S$ - $p$ por ambiente químico $\mathrm{Cu}-\mathrm{O} 2$ y M-O1 (ver Figura 7-1), de los sistemas a) $\mathrm{Li}_{2} \mathrm{Cu}_{0.5} \mathrm{Co}_{0.5} \mathrm{O}_{2}$, b) $\mathrm{Li}_{2} \mathrm{Cu}_{0.5} \mathrm{Ni}_{0.5} \mathrm{O}_{2}$ y c) $\mathrm{Li}_{2} \mathrm{Cu}_{0.5} \mathrm{Ti}_{0.5} \mathrm{O}_{2}$, obtenidas con el funcional HSE06. La línea punteada de color negro indica el nivel de Fermi en cada sistema. El nivel de Fermi y la línea punteada de color violeta encierran el intervalo de energía donde la DOS integra a 1 electrón.

\subsection{Análisis de la blandura local}

Uno de los objetivos de este trabajo fue determinar si la blandura local, $s(\mathbf{r})$, siendo un coeficiente de respuesta, podría servir como una herramienta para el estudio del proceso redox del $\mathrm{Li}_{2} \mathrm{CuO}_{2}, \mathrm{LiCuO}_{2}$ y los sistemas $\mathrm{Li}_{2} \mathrm{Cu}_{0.5} \mathrm{M}_{0.5} \mathrm{O}_{2}$. Por ello, se determinó la $s(\mathbf{r})$ para cada uno de estos sistemas, bajo la condición descrita en la ecuación 6-7. Los resultados son mostrados en la Figura 7-7 y Figura 7-8, donde se visualizan los sitios susceptibles a 
la sustracción de un electrón en cada uno de los sistemas. Las conclusiones obtenidas por el análisis de estos datos son consistentes con el análisis de la DOS-p.

\subsection{1 $\mathrm{Li}_{2} \mathrm{CuO}_{2}$ y $\mathrm{LiCuO}_{2}$}

Las $s(\mathbf{r})$ obtenidas con el funcional HSE06 correspondientes a $\mathrm{Li}_{2} \mathrm{CuO}_{2}$ y $\mathrm{LiCuO}_{2}$ son mostradas en la Figura 7-7. En el caso de $\mathrm{Li}_{2} \mathrm{CuO}_{2}$, se aprecia que las regiones susceptibles al retiro de un electrón corresponden a sitios sobre los átomos de $\mathrm{Cu}$ y $\mathrm{O}$, siendo en estos últimos la región con más concentración del isovalor graficado, por lo que se concluye que los átomos de $\mathrm{O}$ y $\mathrm{Cu}$ participan en el proceso redox de este sistema, siendo los átomos de O los más susceptibles a oxidarse, ocasionando una mayor disposición a la liberación de oxígeno. Posteriormente si el sistema es parcialmente deslitiado, es posible obtener el sistema $\mathrm{LiCuO}_{2}$, en el cual, tal y como se muestra en la Figura 7-7, la susceptibilidad de los átomos de $\mathrm{O}$ a ser oxidados aumenta, por lo que la disposición a la liberación de oxígeno también es de esperarse que incremente, lo cual ya ha sido planteado previamente tanto para $\mathrm{Li}_{2} \mathrm{CuO}_{2}$ como para $\mathrm{LiCuO}_{2}$ [24].

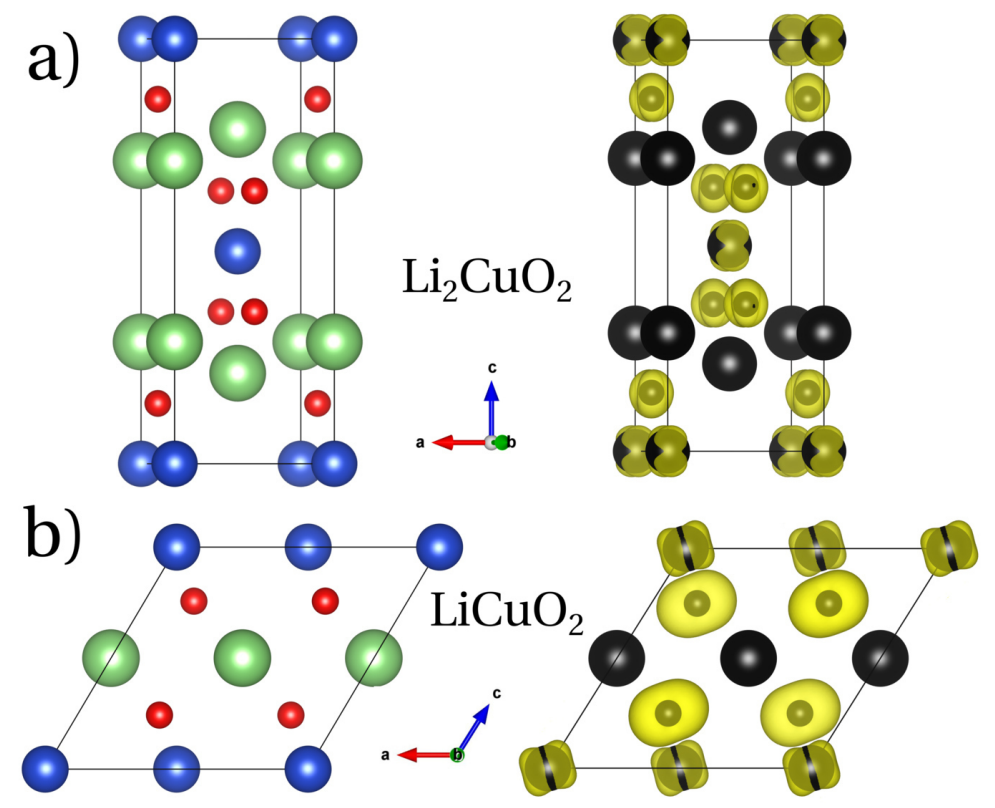

Figura 7-7: Gráficas de isosuperficies de $s(\mathbf{r})$ para los sistemas a) $\mathrm{Li}_{2} \mathrm{CuO}_{2}$ y b) $\mathrm{LiCuO}_{2}$. Se utilizó un isovalor de 0.354 unidades atómicas (en color amarillo, a la derecha), seleccionado de tal manera que permitió la comparación de la $s(\mathbf{r})$ entre $\mathrm{Li}_{2} \mathrm{CuO}_{2}, \mathrm{LiCuO}_{2}$ y Li $\mathrm{Cu}_{1-x} \mathrm{M}_{x} \mathrm{O}_{2}$. Las esferas color verde representan los átomos de litio, las azules átomos de cobre y las rojas oxígenos. 


\subsubsection{Sistemas del tipo $\mathrm{Li}_{2} \mathrm{Cu}_{0.5} \mathrm{M}_{0.5} \mathrm{O}$}

En la Figura 7-8 se muestran las gráficas de $s(\mathbf{r})$ para los sistemas $\mathrm{Li}_{2} \mathrm{Cu}_{0.5} \mathrm{M}_{0.5} \mathrm{O}_{2}(\mathrm{M}$ $=$ Co, $\mathrm{Ni}$ o Ti), comparadas con la $s(\mathbf{r})$ de $\mathrm{Li}_{2} \mathrm{CuO}_{2}$. Puede notarse que en el caso de $\mathrm{Li}_{2} \mathrm{Cu}_{0.5} \mathrm{Co}_{0.5} \mathrm{O}_{2}$, las regiones susceptibles al retiro de un electrón, se sitúan en mayor medida sobre el átomo de Co y los átomos de $\mathrm{O}$ más cercanos, es decir, sobre los cuadrados Co-O1. La susceptibilidad de los $\mathrm{O}$ tipo 2 , los más cercanos a $\mathrm{Cu}$, es menor en comparación con $\mathrm{Li}_{2} \mathrm{CuO}_{2}$, por lo que la posibilidad de liberación de oxígeno es menor que en $\mathrm{Li}_{2} \mathrm{CuO}_{2}$, lo cual puede repercutir positivamente a que este sistema presente una mayor retención de la capacidad durante ciclos repetidos de carga-descarga, resultado que ha sido reportado experimentalmente para un sistema similar, $\mathrm{Li}_{2} \mathrm{Cu}_{0.9} \mathrm{Co}_{0.1} \mathrm{O}_{2}$ [20]. En el caso de $\mathrm{Li}_{2} \mathrm{Cu}_{0.5} \mathrm{Ni}_{0.5} \mathrm{O}_{2}$, el efecto es similar al obtenido para $\mathrm{Li}_{2} \mathrm{Cu}_{0.5} \mathrm{Co}_{0.5} \mathrm{O}_{2}$ pero en menor grado y con la participación de sitios sobre Cu. También es de esperarse que este sistema contribuya a una menor participación redox durante el proceso de carga-descarga y poder así aumentar la retención de la capacidad. Experimentalmente esto se ha observado en los sistemas $\mathrm{Li}_{2} \mathrm{Cu}_{0.7} \mathrm{Ni}_{0.3} \mathrm{O}_{2} \quad$ [20] y $\mathrm{Li}_{2} \mathrm{Cu}_{0.5} \mathrm{Ni}_{0.5} \mathrm{O}_{2} \quad$ [16]. Cabe mencionar que la comparación con los resultados experimentales, no puede hacerse directamente debido a que se trabaja con modelos simples donde la proporción Cu:M ( $\mathrm{M}=$ Co o Ni) así como el arreglo estructural son diferentes; pero es posible mencionar que el efecto de incluir átomos de Co o $\mathrm{Ni}$ en la estructura cristalina de $\mathrm{Li}_{2} \mathrm{CuO}_{2}$ resulta en una disminución en la participación redox de oxígeno.

La $s(\mathbf{r})$ de $\mathrm{Li}_{2} \mathrm{Cu}_{0.5} \mathrm{Ti}_{0.5} \mathrm{O}_{2}$ muestra que las regiones susceptibles a oxidarse por la sustracción de un electrón, radican sobre el átomo de Ti, estando ausente la participación de $\mathrm{O}$, por lo que es de esperarse que un sistema cristalino $\mathrm{Li}_{2} \mathrm{CuO}_{2}$ donde parte de sus átomos de $\mathrm{Cu}$ sean sustituidos por átomos $\mathrm{Ti}$, presente una mayor retención de la capacidad (en comparación con $\mathrm{Li}_{2} \mathrm{CuO}_{2}$ ) debido a una disminución considerable de la participación redox de oxígeno y la participación redox exclusiva del Ti en el proceso de carga-descarga de $\mathrm{Li}_{2} \mathrm{Cu}_{0.5} \mathrm{Ti}_{0.5} \mathrm{O}_{2}$.

Es relevante mencionar que estas propuestas se hacen con sólo observar la gráfica de la $s(\mathbf{r})$, sin la necesidad de hacer una proyección sobre átomos (para obtener la DOS- $p$ ) y posteriormente agrupar esto por coordinación ( $\mathrm{Cu}-\mathrm{O}$ y $\mathrm{M}-\mathrm{O})$. En un sólo paso se obtiene la misma conclusión, por lo que se propone que desde el punto de vista práctico así como el visual, la $s(\mathbf{r})$ resulta ser un índice de reactividad ad hoc a este fenómeno que, no sólo permite hacer un análisis cualitativo sino también cuantitativo, ya que puede integrarse por región atómica y dar un número (cantidad) sobre cada átomo, de forma muy similar a lo que se obtiene al trabajar con las funciones de Fukui condensadas [103]. 


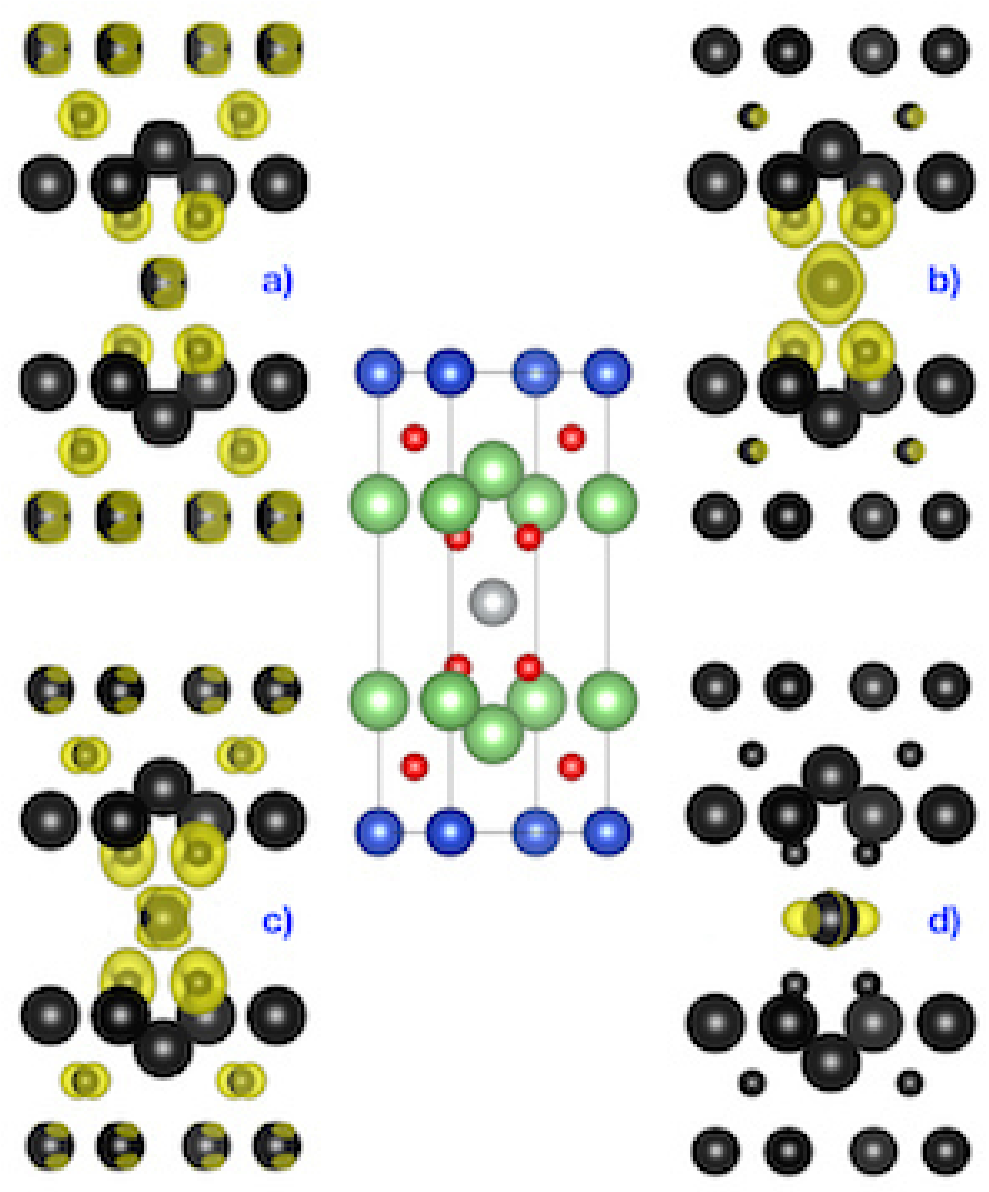

Figura 7-8: Gráficas de isosuperficies de $s(\mathbf{r})$ con un valor de 0.354 unidades atómicas, para los sistemas a) $\mathrm{Li}_{2} \mathrm{CuO}_{2}$, b) $\mathrm{Li}_{2} \mathrm{Cu}_{0.5} \mathrm{Co}_{0.5} \mathrm{O}_{2}$, c) $\mathrm{Li}_{2} \mathrm{Cu}_{0.5} \mathrm{Ni}_{0.5} \mathrm{O}_{2}$ y d) $\mathrm{Li}_{2} \mathrm{Cu}_{0.5} \mathrm{Ti}_{0.5} \mathrm{O}_{2}$. En el centro se muestra la orientación espacial de la celda unitaria. Las esferas color verde representan los átomos de litio, las azules átomos de cobre, las rojas oxígenos y la gris representa al átomos $\mathrm{M}=\mathrm{Cu}$, Co, Ni o Ti.

\subsection{Blandura local con otros funcionales de intercambio-correlación}

De la sección anterior puede notarse que la blandura local resulta práctica para el conocimiento de los sitios más afectados ante la sustracción de un electrón. Por ello el propósito de esta sección fue determinar el comportamiento de la blandura local al utilizar diferentes aproximaciones al funcional de intercambio-correlación, con el fin de conocer si este índice de reactividad es sensible a la aproximación utilizada en el funcional de intercambiocorrelación, tal y como sucede en la obtención de la DOS- $p$. 


\subsubsection{Sistemas $\mathrm{Li}_{2} \mathrm{CuO}_{2}$ y $\mathrm{LiCuO}_{2}$}

En la Figura 7-9 se muestran las gráficas de la $s(\mathbf{r})$ para $\mathrm{Li}_{2} \mathrm{CuO}_{2}$ y $\mathrm{LiCuO}_{2}$ contenidas con diferentes aproximaciones al funcional de intercambio-correlación. En esta se muestra que las $s(\mathbf{r})$ obtenidas con HSE06 son muy similares a las obtenidas con PBE $+\mathrm{U}$ (para ambos sistemas). En el caso de $\mathrm{LiCuO}_{2}$, la principal diferencia entre HSE06 y PBE+U radica en la forma de las regiones sobre los átomos de $\mathrm{O}$, pero ambas aproximaciones dejan claro la misma interpretación: los sitios más afectados por la sustracción de un electrón son aquellos de oxígeno y en menor proporción los sitios sobre los átomos de $\mathrm{Cu}$. Esta distinción en relación a mayor susceptibilidad de los sitios de $\mathrm{O}$ respecto de los de $\mathrm{Cu}$, también puede ser observada pero en menor grado en los resultados obtenidos con PBE, PBE-sol y SCAN, donde se aprecia una región más grande sobre los átomos de $\mathrm{Cu}$ en comparación con las regiones obtenidas con HSE06 y PBE+U. Un resultado y discusión similares pueden obtenerse en el caso de $\mathrm{Li}_{2} \mathrm{CuO}_{2}$, donde las $s(\mathbf{r})$ obtenidas con HSE06 y PBE $+\mathrm{U}$ muestran regiones más grandes sobre los átomos de $\mathrm{O}$ que los de $\mathrm{Cu}$, lo cual no es observado en los resultados obtenidos con PBE, PBE-sol y SCAN. Con base en estos resultados, se puede concluir que al agregar correcciones para la auto-interacción electrónica (como en el caso del funcional de rango separado HSE06 o la aproximación PBE+U) permite diferenciar los sitios más reactivos a la sustracción de un electrón en el caso de $\mathrm{Li}_{2} \mathrm{CuO}_{2} \mathrm{y} \mathrm{LiCuO}_{2}$; mientras que en casos de no agregar esta corrección (como en PBE, PBE-sol y SCAN) esta observación no se puede hacer. 


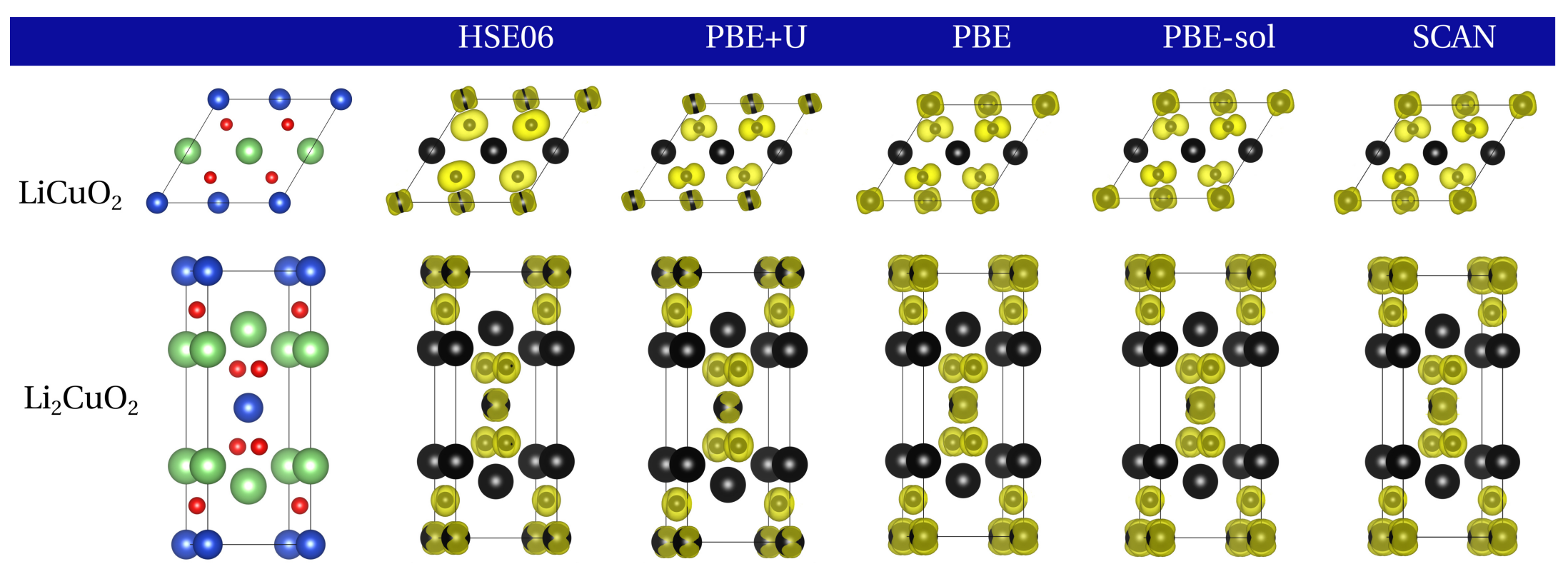

Figura 7-9: Comparación de la $s(\mathbf{r})$ para $\mathrm{LiCuO}_{2}$ y $\mathrm{Li}_{2} \mathrm{CuO}_{2}$ obtenida con diferentes funcionales de intercambio-correlación. Se utilizó un isovalor de 0.354 unidades atómicas, seleccionado de tal manera que permitió la comparación de la $s(\mathbf{r})$ entre $\mathrm{Li}_{2} \mathrm{CuO}_{2}, \mathrm{LiCuO}_{2}$ y $\mathrm{Li}_{2} \mathrm{Cu}_{1-x} \mathrm{M}_{x} \mathrm{O}_{2}$. Las esferas color verde representan los átomos de litio, las azules átomos de cobre y las rojas oxígenos. 


\subsubsection{Sistemas del tipo $\mathrm{Li}_{2} \mathrm{Cu}_{0.5} \mathrm{IM}_{0.5} \mathrm{O}$}

En este apartado hacemos un análisis similar al llevado a cabo en la sección anterior pero con los resultados obtenidos para $\mathrm{Li}_{2} \mathrm{Cu}_{0.5} \mathrm{Co}_{0.5} \mathrm{O}_{2}, \mathrm{Li}_{2} \mathrm{Cu}_{0.5} \mathrm{Ni}_{0.5} \mathrm{O}_{2}$ y $\mathrm{Li}_{2} \mathrm{Cu}_{0.5} \mathrm{Ti}_{0.5} \mathrm{O}_{2}$, los cuales son mostrados en la Figura 7-10. De la sección 7.5.2 se concluyó que, en el caso de $\mathrm{Li}_{2} \mathrm{Cu}_{0.5} \mathrm{Co}_{0.5} \mathrm{O}_{2}$, a partir de la $s(\mathbf{r})$ obtenida (con PBE+U o HSE06), el efecto de incluir Co en la estructura cristalina del $\mathrm{Li}_{2} \mathrm{CuO}_{2}$ es disminuir la susceptibilidad de los átomos de $\mathrm{O}$ a la sustracción de un electrón, particularmente aquellos átomos de $\mathrm{O}$ que están coordinados a $\mathrm{Cu}$, siendo los cuadrados de coordinación Co-O los más reactivos al retiro de un electrón. Esta misma observación puede hacerse con las $s(\mathbf{r})$ obtenidas con los funcionales PBE y PBE-sol; sin embargo, no es posible concluir lo mismo con el resultado obtenido con el funcional SCAN, donde parece que los planos cuadrados $\mathrm{Cu}-\mathrm{O}$, son los más propensos a ser oxidados.

Cuando se comparan los resultados obtenidos en el caso de $\mathrm{Li}_{2} \mathrm{Cu}_{0.5} \mathrm{Ni}_{0.5} \mathrm{O}_{2}$ se concluye que las cinco aproximaciones proponen que los cuadrados $\mathrm{Ni}-\mathrm{O}$ son los más reactivos a ser oxidados, los cuales son los únicos reactivos en el caso de PBE y PBE-sol y en el caso de SCAN, parece ser que ambos planos cuadrados ( $\mathrm{Cu}-\mathrm{O}$ y Ni-O) son casi igualmente susceptibles.

En el caso del $\mathrm{Li}_{2} \mathrm{Cu}_{0.5} \mathrm{Ti}_{0.5} \mathrm{O}_{2}$, el resultado obtenido con HSE06 deja claro que el átomo de Ti es el único susceptible a participar en el proceso redox, en el caso de PBE+U no sólo es el átomo de Ti sino además participan los átomos de $\mathrm{Cu}$, en menor medida, los átomos de O coordinados a éste. Lo mismo en el caso de los resultados obtenidos con PBE, PBE-sol y SCAN pero con mayor grado. A pesar de estas diferencias se observa un común denominador en los resultados obtenidos con estas cinco aproximaciones: el efecto de incluir Ti en la estructura del $\mathrm{Li}_{2} \mathrm{CuO}_{2}$ resulta en disminuir la susceptibilidad de los átomos de $\mathrm{O}$ a ser oxidados en el proceso de carga, siendo este efecto más marcado en los $O$ coordinados a éste que aquellos coordinados a $\mathrm{Cu}^{\mathrm{h}}$.

Con estos resultados podemos resumir que en la mayoría de los casos (con excepción de $\mathrm{Li}_{2} \mathrm{Cu}_{0.5} \mathrm{Ti}_{0.5} \mathrm{O}_{2}$ ), los resultados y observaciones obtenidos a partir de HSE06 y PBE+U resultan ser consistentes. Sin embargo, estas observaciones no siempre pueden ser establecidas con los resultados obtenidos con PBE, PBE-sol y SCAN, por lo que es concluyente que la $s(\mathbf{r})$ sí es sensible al funcional XC utilizado.

\footnotetext{
hEsto puede relacionarse al hecho de que el $\mathrm{Ti}^{2+}$ es un estado muy inestable y fuertemente reductor [96].
} 


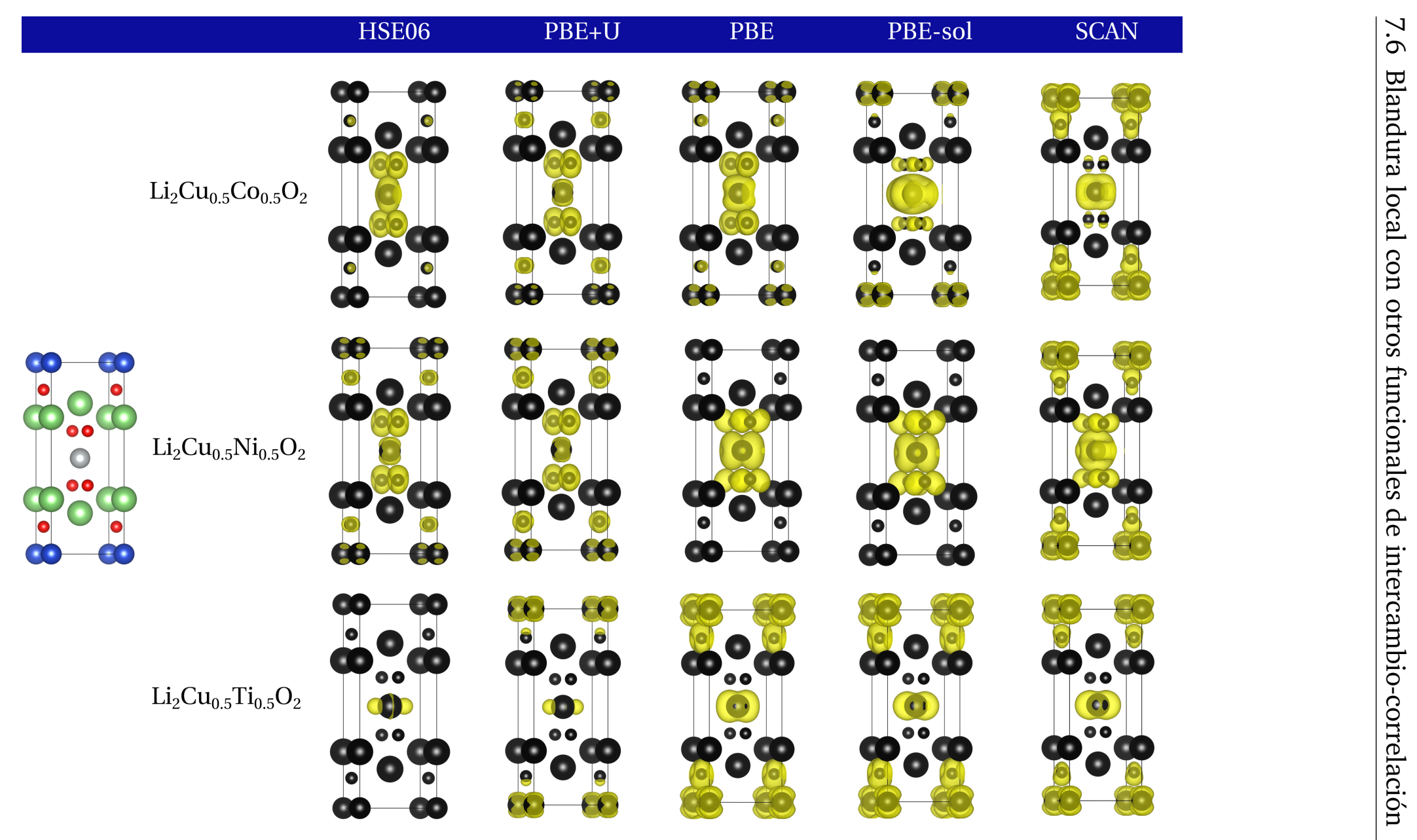

Figura 7-10: Comparación de la $s(\mathbf{r})$ para $\mathrm{Li}_{2} \mathrm{Cu}_{0.5} \mathrm{Co}_{0.5} \mathrm{O}_{2}, \mathrm{Li}_{2} \mathrm{Cu}_{0.5} \mathrm{Ni}_{0.5} \mathrm{O}_{2}$ y Li $\mathrm{Cu}_{0.5} \mathrm{Ti}_{0.5} \mathrm{O}_{2}$ obtenida con diferentes funcionales de intercambio-correlación. Se utilizó un isovalor de 0.354 unidades atómicas. Las esferas color verde representan los átomos de litio, las azules átomos de cobre, las rojas oxígenos y la gris representa a los átomos $\mathrm{M}=\mathrm{Cu}$, Co, Ni o Ti. 


\subsection{Voltaje de inserción de litio en $\mathrm{LiCuO}_{2}, \mathrm{Li}_{2} \mathrm{CuO}_{2}$ y $\mathrm{Li}_{2} \mathrm{Cu}_{0.5} \mathrm{M}_{0}$}

En la Tabla 7-5 se muestra el voltaje teórico promedio de inserción de litio para los sistemas $\mathrm{Li}_{2} \mathrm{CuO}_{2}$ y Li $\mathrm{Cu}_{0.5} \mathrm{M}_{0.5} \mathrm{O}_{2}$ de acuerdo a las reacciones (6-15) y (6-16), obtenidos con $\mathrm{PBE}+\mathrm{U}$ y HSE06. Los voltajes obtenidos con HSE06 son más altos que los obtenidos con $\mathrm{PBE}+\mathrm{U}$, en todos los casos. Al comparar los voltajes experimentales con los teóricos (en el caso de $\mathrm{Li}_{2} \mathrm{CuO}_{2}$ y Li ${ }_{2} \mathrm{Cu}_{0.5} \mathrm{Ni}_{0.5} \mathrm{O}_{2}$ ), se observa que, tanto PBE+U como HSE06 subestiman el voltaje. Sin embargo la tendencia de que $\mathrm{Li}_{2} \mathrm{CuO}_{2}$ posee un mayor voltaje experimental que $\mathrm{Li}_{2} \mathrm{Cu}_{0.5} \mathrm{Ni}_{0.5} \mathrm{O}_{2}$ se mantiene tanto en los voltajes obtenidos con PBE+U como los de HSE06. En otros materiales se ha obtenido un comportamiento similar, es decir, HSE06 produce voltajes más altos que $\mathrm{PBE}+\mathrm{U}$ [81].

En orden descendente de acuerdo a su voltaje promedio de inserción de litio, los sistemas $\mathrm{Li}_{2} \mathrm{CuO}_{2}$ y Li $\mathrm{Cu}_{0.5} \mathrm{M}_{0.5} \mathrm{O}_{2}$ siguen la tendencia $\mathrm{Li}_{2} \mathrm{CuO}_{2}>\mathrm{Li}_{2} \mathrm{Cu}_{0.5} \mathrm{Co}_{0.5} \mathrm{O}_{2}>\mathrm{Li}_{2} \mathrm{Cu}_{0.5} \mathrm{Ni}_{0.5} \mathrm{O}_{2}$ $>\mathrm{Li}_{2} \mathrm{Cu}_{0.5} \mathrm{Ti}_{0.5} \mathrm{O}_{2}$, la cual es la misma que la de los potenciales de los pares redox metálicos en solución acuosa [104]: $\mathrm{Cu}^{3+} / \mathrm{Cu}^{2+}(2.4 \mathrm{~V})>\mathrm{Co}^{3+} / \mathrm{Co}^{2+}(2.3 \mathrm{~V})>\mathrm{Ni}^{3+} / \mathrm{Ni}^{2+}(1.92 \mathrm{~V})$ $>\mathrm{Ti}^{3+} / \mathrm{Ti}^{2+}(0.9 \mathrm{~V})$.

Tabla 7-5: Voltajes de inserción de litio (en volts) calculados con PBE+U y HSE06, valores experimentales son mostrados entre paréntesis.

\begin{tabular}{cccc}
\hline Sistema & \multicolumn{3}{c}{ Voltaje } \\
& PBE+U & HSE06 & Experimental \\
\hline $\mathbf{L i}_{2} \mathbf{C u O} \mathbf{O}_{2}$ & 3.01 & 3.28 & $(3.4)^{\mathrm{a}}$ \\
$\mathbf{L i}_{2} \mathbf{C u}_{0.5} \mathbf{C o}_{0.5} \mathbf{O}_{2}$ & 2.77 & 2.90 & - \\
$\mathbf{L i}_{2} \mathbf{C u}_{0.5} \mathbf{N i}_{0.5} \mathbf{O}_{2}$ & 2.71 & 2.79 & $(3.1-3.2)^{\mathrm{b}}$ \\
$\mathbf{L i}_{2} \mathbf{C u}_{0.5} \mathbf{T i}_{0.5} \mathbf{O}_{2}$ & 1.31 & 1.44 & - \\
\hline \hline
\end{tabular}

${ }^{a}$ Potencial del primer proceso de deslitiación de Ref [18]

b Potencial del primer proceso de deslitiación de Ref [16]

\subsection{Sistemas del tipo $\mathrm{Li}_{2} \mathrm{Cu}_{0.75} \mathrm{M}_{0.25} \mathrm{O}_{2}$}

\subsubsection{Densidad de estados proyectada de los sistemas $\mathrm{Li}_{2} \mathrm{Cu}_{0.75} \mathrm{M}_{0.25} \mathrm{O}_{2}$}

Hasta el momento, toda la discusión realizada en torno al efecto de incluir otros metales de transición a la estructura cristalina de $\mathrm{Li}_{2} \mathrm{CuO}_{2}$, ha sido utilizando modelos en los cuales las láminas formadas por metales de transición son homogéneas, es decir, cada una de ellas sólo posee un tipo de metal de transición ( $\mathrm{Cu}$ o $\mathrm{M}=\mathrm{Co}$, Ni o Ti). Sin embargo, durante el proceso de síntesis de este tipo de sistemas, es posible que la sustitución de los átomos de $\mathrm{Cu}$ por $\mathrm{M}$, en la red cristalina de $\mathrm{Li}_{2} \mathrm{CuO}_{2}$ sea de forma tal que se genere desorden catióni- 
co, es decir, que cada una de las láminas de metal de transición estén compuestas tanto de $\mathrm{Cu}$ como de $\mathrm{M}$, como en el caso de $\mathrm{Li}_{2} \mathrm{Cu}_{0.5} \mathrm{Ni}_{0.5} \mathrm{O}_{2}$ [16]. Por esta razón se construyeron los sistemas $\mathrm{Li}_{2} \mathrm{Cu}_{0.75} \mathrm{M}_{0.25} \mathrm{O}_{2}$ ( $\mathrm{M}=\mathrm{Co}$, Ni o Ti) de los cuales, la DOS- $p$ de cada uno es mostrada en la Figura 7-11, las cuales fueron obtenidas con el funcional HSE06. En el caso de los sistemas $\mathrm{Li}_{2} \mathrm{Cu}_{0.75} \mathrm{Co}_{0.25} \mathrm{O}_{2}$ y Li $\mathrm{Cu}_{0.75} \mathrm{Ni}_{0.25} \mathrm{O}_{2}$, los estados ocupados cercanos al nivel de Fermi (región de interés, delimitada por las líneas punteadas de color negro y morado en la Figura 7-11) corresponden en mayor parte a estados pertenecientes a oxígeno, en segundo lugar por los estados del metal sustituyente $\mathrm{M}$ (Co o Ni) y por último, los estados correspondientes a $\mathrm{Cu}$. Ambos sistemas exhiben un gap similar $(2.58 \mathrm{eV}$ y $2.51 \mathrm{eV}$ para $\mathrm{Li}_{2} \mathrm{Cu}_{0.75} \mathrm{Co}_{0.25} \mathrm{O}_{2}$ y $\mathrm{Li}_{2} \mathrm{Cu}_{0.75} \mathrm{Ni}_{0.25} \mathrm{O}_{2}$, respectivamente) mientras que el comportamiento de los primeros estados vacíos es distinto. En el caso de $\mathrm{Li}_{2} \mathrm{Cu}_{0.75} \mathrm{Co}_{0.25} \mathrm{O}_{2}$, los estados vacíos más próximos al nivel de Fermi corresponden en su mayoría a $\mathrm{Cu}$ y en segundo lugar a $\mathrm{O}$; mientras que en el caso de $\mathrm{Li}_{2} \mathrm{Cu}_{0.75} \mathrm{Ni}_{0.25} \mathrm{O}_{2}$, los estados correspondientes a $\mathrm{Ni}$ son los mayoritarios y más propensos a recibir electrones.

Al igual que en el caso de $\mathrm{Li}_{2} \mathrm{Cu}_{0.5} \mathrm{M}_{0.5} \mathrm{O}_{2}$, el sistema en el cual $\mathrm{M}=\mathrm{Ti}\left(\mathrm{Li}_{2} \mathrm{Cu}_{0.75} \mathrm{Ti}_{0.25} \mathrm{O}_{2}\right)$, muestra un comportamiento diferente. Los estados ocupados más susceptibles a ser oxidados son los de Ti seguidos por aquellos que pertenecen a Cu. Nuevamente el efecto de incluir $\mathrm{Ti}$ en la estructura de $\mathrm{Li}_{2} \mathrm{CuO}_{2}$ (en un arreglo y proporción diferentes) es disminuir la contribución de los estados de oxígeno cerca del nivel de Fermi. Esto es lo que se puede concluir hasta aquí, únicamente analizando la DOS- $p$, sin embargo, al visualizar la $s(\mathbf{r})$, se puede obtener mayor información, como se analiza a continuación. 

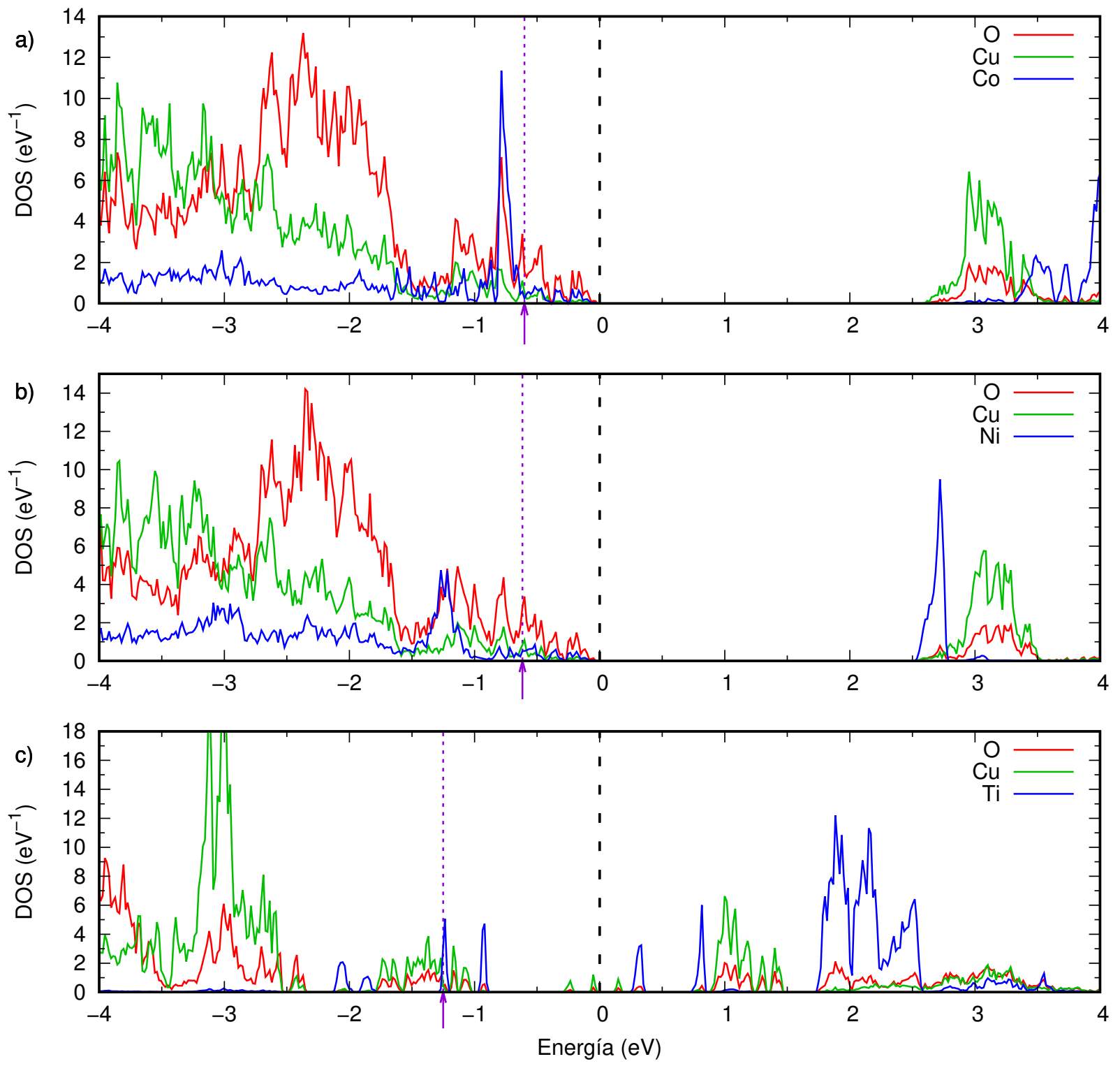

Figura 7-11: DOS-p de a) $\mathrm{Li}_{2} \mathrm{Cu}_{0.75} \mathrm{Co}_{0.25} \mathrm{O}_{2}$, b) $\mathrm{Li}_{2} \mathrm{Cu}_{0.75} \mathrm{Ni}_{0.25} \mathrm{O}_{2}$ y c) $\mathrm{Li}_{2} \mathrm{Cu}_{0.75} \mathrm{Ti}_{0.25} \mathrm{O}_{2}$, obtenidas con el funcional HSE06. La línea punteada de color negro indica el nivel de Fermi de cada sistema. El nivel de Fermi y la línea punteada de color violeta encierran el rango de energía donde la DOS integra a 1 electrón. 


\subsubsection{Blandura local de los sistemas $\mathrm{Li}_{2} \mathrm{Cu}_{0.75} \mathrm{M}_{0.25} \mathrm{O}_{2}$}
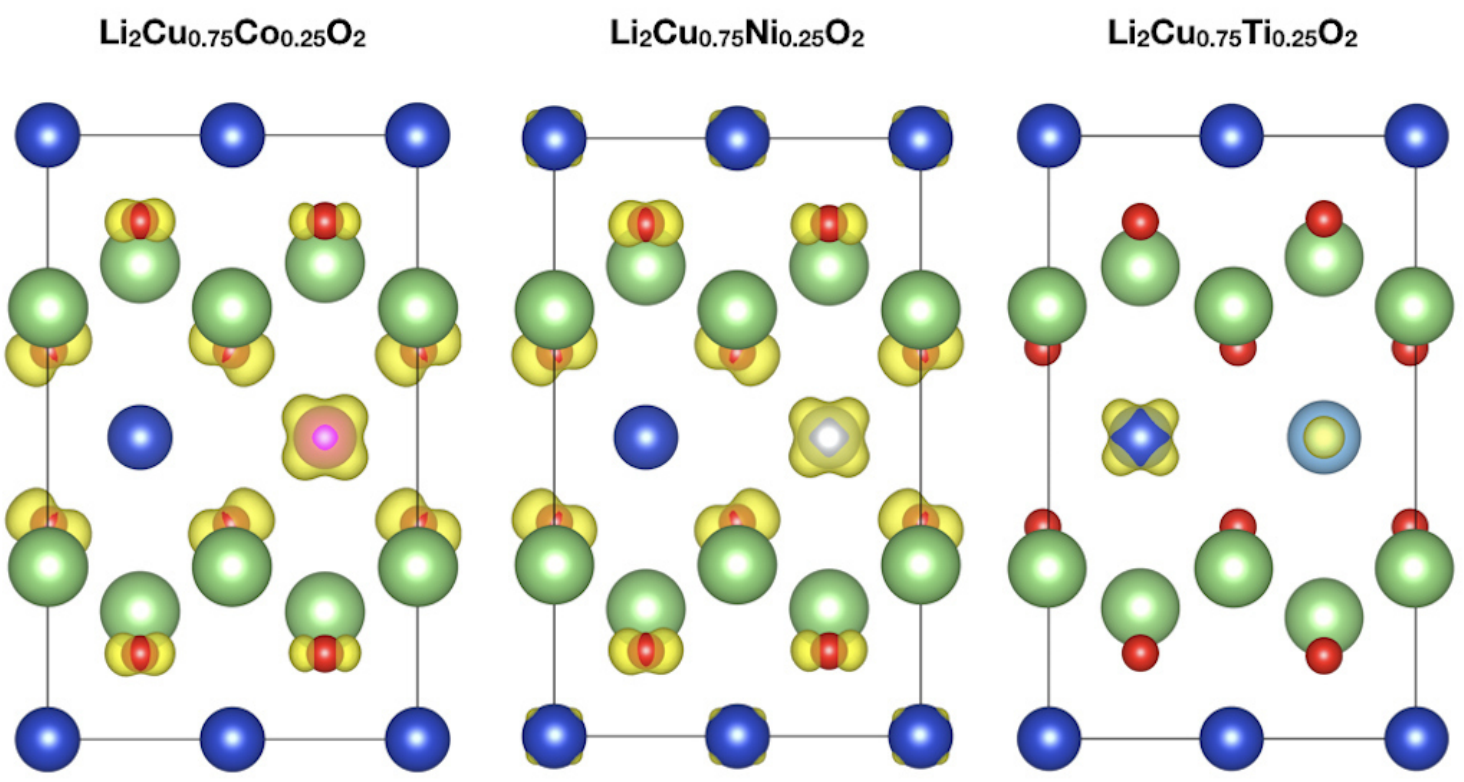

Figura 7-12: Gráficas de isosuperficies de $s(\mathbf{r})$ con un isovalor de 0.354 unidades atómicas(en color amarillo), para los sistemas $\mathrm{Li}_{2} \mathrm{Cu}_{0.75} \mathrm{M}_{0.25} \mathrm{O}_{2}$ ( $\mathrm{M}=\mathrm{Co}$, Ni o Ti), obtenidas con HSE06. Las esferas color verde representan los átomos de litio, las azules átomos de cobre, las rojas oxígenos, la rosa, gris y azul celeste representan átomos de cobalto, níquel y titanio, respectivamente.

En el apartado precedente sólo pudo mencionarse que, en los sistemas $\mathrm{Li}_{2} \mathrm{Cu}_{0.75} \mathrm{Co}_{0.25} \mathrm{O}_{2} \mathrm{y}$ $\mathrm{Li}_{2} \mathrm{Cu}_{0.75} \mathrm{Ni}_{0.25} \mathrm{O}_{2}$, la contribución de los estados correspondientes a $\mathrm{O}$ es mayoritaria en la región de interés y no resulta tan claro el efecto de Co y $\mathrm{Ni}$ en estos sistemas respeto a la contribución de estados de $\mathrm{O}$ en la región de sustracción de un electrón, como en el caso de $\mathrm{Li}_{2} \mathrm{Cu}_{0.75} \mathrm{Ti}_{0.25} \mathrm{O}_{2}$, donde los estados dominantes son aquellos correspondientes a Ti. Sin embargo, si analizamos las $s(\mathbf{r})$ de cada uno de estos sistemas (Figura 7-12) resulta más evidente este efecto.

En el sistema $\mathrm{Li}_{2} \mathrm{Cu}_{0.75} \mathrm{Co}_{0.25} \mathrm{O}_{2}$, se observa que el efecto de Co es diminuir la susceptibilidad de los átomos de $\mathrm{O}$ coordinados a $\mathrm{Cu}$ en las láminas homogéneas de $\mathrm{Cu}$, lo cual no resulta a primera vista evidente si nos limitamos a analizar sólo la DOS- $p$, es necesario analizar la DOS-p por plano cuadrado, lo cual resulta en una manipulación adicional que nos llevaría al mismo resultado que el obtenido por analizar la $s(\mathbf{r})$ directamente. La misma observación y conclusión se establece en el sistema $\mathrm{Li}_{2} \mathrm{Cu}_{0.75} \mathrm{Ni}_{0.25} \mathrm{O}_{2}$, con la diferencia de que el efecto es menos marcado. Por último, en el caso de $\mathrm{Li}_{2} \mathrm{Cu}_{0.75} \mathrm{Ti}_{0.25} \mathrm{O}_{2}$ (figuras 7-12 y 7-13), el efecto es evidente, la incorporación de Ti a la red conlleva a desaparecer la susceptibilidad de los átomos de $\mathrm{O}$ a ser oxidados y en cambio deja como especies 
propensas a oxidarse a los átomos de $\mathrm{Cu}$ y Ti de la lámina heterogénea $\mathrm{Cu} / \mathrm{Ti}$. Con estos resultados, la conclusión del efecto del metal sustituyente $\mathrm{M}$, es la misma: se disminuye la susceptibilidad de los átomos de $\mathrm{O}$ a oxidarse y en el caso de $\mathrm{Li}_{2} \mathrm{Cu}_{0.75} \mathrm{Ti}_{0.25} \mathrm{O}_{2}$, desaparece. Es relevante adicionar que las proporciones de $\mathrm{M}$ en relación a las de $\mathrm{Cu}$, son menores que en el caso de los sistemas con láminas de metal de transición homogéneas $\left(\mathrm{Li}_{2} \mathrm{Cu}_{0.5} \mathrm{M}_{0.5} \mathrm{O}_{2}\right)$ y, aún así el efecto esperado es el mismo, la disminución de la participación redox del oxígeno en el proceso de carga. De acuerdo a los resultados obtenidos en los sistemas $\mathrm{Li}_{2} \mathrm{Cu}_{0.5} \mathrm{M}_{0.5} \mathrm{O}_{2}$ y Li $\mathrm{Cu}_{0.75} \mathrm{M}_{0.25} \mathrm{O}_{2}$, hacer sustituciones de átomos de $\mathrm{Cu}$ en la estructura cristalina de $\mathrm{Li}_{2} \mathrm{CuO}_{2}$, genera sistemas del tipo $\mathrm{Li}_{2} \mathrm{Cu}_{1-x} \mathrm{M}_{x} \mathrm{O}_{2}$, cuyos oxígenos son menos susceptibles a ser oxidados, específicamente aquellos oxígenos coordinados a $\mathrm{Cu}$. La $s(\mathbf{r})$ mostró las regiones más susceptibles a ser oxidadas, durante el proceso de carga de cada sistema estudiado en este trabajo, sin embargo, puede explorarse su uso en un contexto diferente a las BIL, tal y como se describe en el apéndice 9.2.

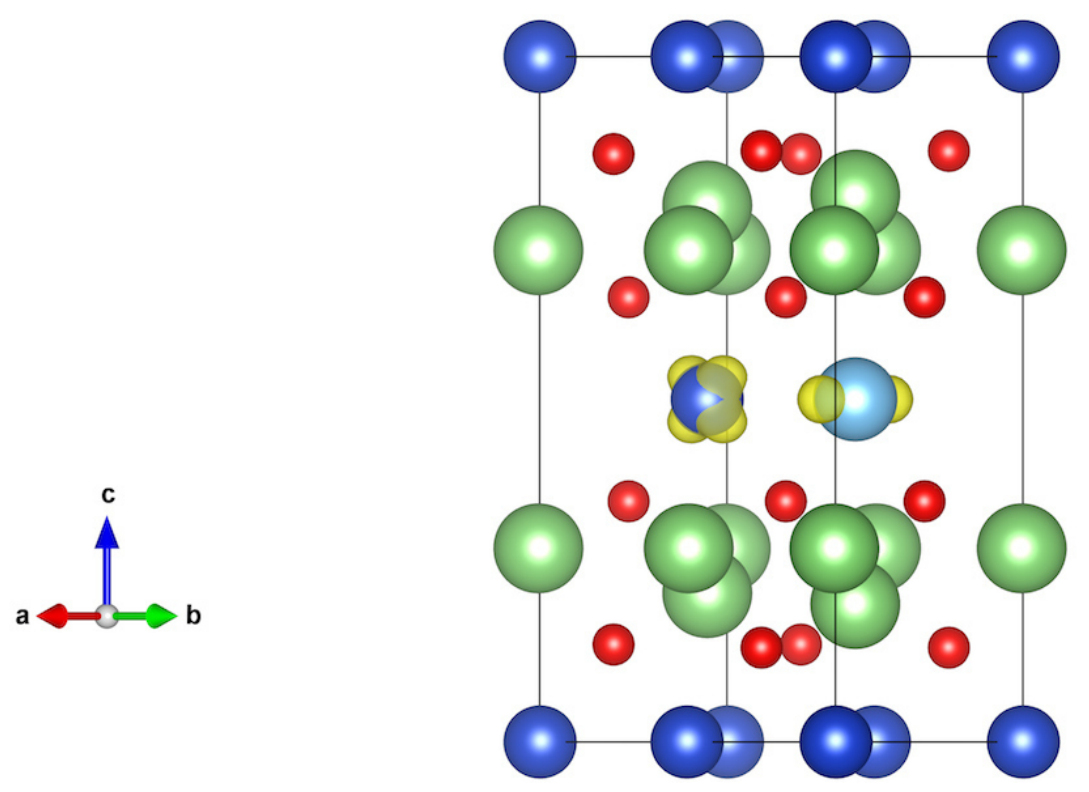

Figura 7-13: Vista lateral de la gráfica de isosuperficie de $s(\mathbf{r})$ con un valor de 0.354 unidades atómicas, para el sistema $\mathrm{Li}_{2} \mathrm{Cu}_{0.75} \mathrm{Ti}_{0.25} \mathrm{O}_{2}$, obtenida con HSE06. 


\section{Capítulo \\ 8

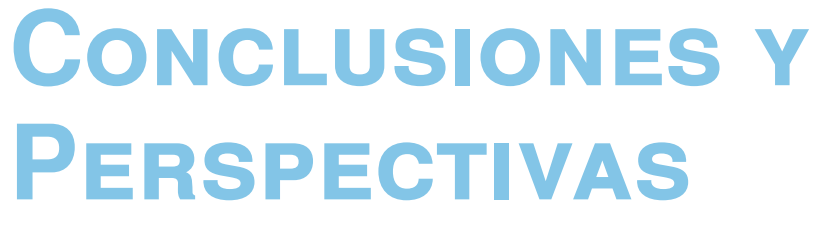

\section{$\mathbf{8 . 1}$ Conclusiones}

La liberación de oxígeno es un factor que influye en la seguridad y desempeño de los materiales catódicos basados en óxidos metálicos en las BIL [24]. En el proceso de carga, la participación redox de oxígeno en $\mathrm{Li}_{2} \mathrm{CuO}_{2}$, ha sido relacionada con: 1) el cambio de la densidad electrónica después de la sustracción de carga y 2) la contribución de oxígeno en los estados cercanos al nivel de Fermi. Se ha mostrado que al retirar un electrón de $\mathrm{Li}_{2} \mathrm{CuO}_{2}$ y restar las densidades electrónicas, los sitios afectados corresponden al plano cuadrado de $\mathrm{CuO}_{4}$ (Figura 2-4) [8,24]. Por otro lado, el análisis de la densidad de estados proyectada (projected density of states, DOS- $p$ ) de $\mathrm{Li}_{2} \mathrm{CuO}_{2}$ y LiCuO${ }_{2}$, sugiere que el estado desocupado por la deslitiación corresponde en mayor medida a oxígeno (Figura 2-5) [8,24], pero no es sencillo hacer una comparación relativa directa.

La relajación estructural de $\mathrm{Li}_{2} \mathrm{CuO}_{2}$ y $\mathrm{LiCuO}_{2}$, mediante la aproximación $\mathrm{PBE}+\mathrm{U}$ predice parámetros de red con sobrestimaciones menores al $3 \%$ respecto al valor experimental y por ello esta aproximación se concluye ser adecuada para estos sistemas. El mayor efecto de la sustitución de un átomo de $\mathrm{Cu}$ por $\mathrm{M}$ en los parámetros estructurales, fue, en el caso de $\mathrm{Li}_{2} \mathrm{Cu}_{0.5} \mathrm{Ti}_{0.5} \mathrm{O}_{2}$, donde el resultado general fue un volumen $6.2 \%$ mayor que $\mathrm{Li}_{2} \mathrm{CuO}_{2}$. Respecto al efecto sobre las distancias de enlace, no existe una tendencia clara, pero vale la pena mencionar nuevamente que en el $\mathrm{Li}_{2} \mathrm{Cu}_{0.5} \mathrm{Ti}_{0.5} \mathrm{O}_{2}$, el efecto del átomo de Ti fue que las distancias Li1-O2 y Li2-O2 fueran ligeramente inferiores en comparación a las de $\mathrm{Li}_{2} \mathrm{CuO}_{2}$.

De acuerdo con los resultados obtenidos de la población electrónica de los orbitales $d$ en cada uno de los metales de transición, así como de la magnetización atómica y global, se concluye que, con excepción de $\mathrm{LiCuO}_{2}$, todos los átomos metálicos de transición, $\mathrm{Cu}, \mathrm{Co}, \mathrm{Ni}$ y Ti, presentan un estado de oxidación $2+$. Los sistemas $\mathrm{Li}_{2} \mathrm{Cu}_{0.5} \mathrm{Co}_{0.5} \mathrm{O}_{2}$ y $\mathrm{Li}_{2} \mathrm{Cu}_{0.5} \mathrm{Ni}_{0.5} \mathrm{O}_{2}$ presentan un esquema de bajo espín en cada uno de sus átomos metálicos, y presentan un ordenamiento antiferromagnético, mientras que en el caso de $\mathrm{Li}_{2} \mathrm{Cu}_{0.5} \mathrm{Ti}_{0.5} \mathrm{O}_{2}$, el ordenamiento es ferromagnético. 
Los voltajes de inserción de litio de los sistemas $\mathrm{Li}_{2} \mathrm{CuO}_{2}$ y $\mathrm{Li}_{2} \mathrm{Cu}_{0.5} \mathrm{M}_{0.5} \mathrm{O}_{2}$ siguen un orden de acuerdo con el potencial de los pares redox en solución acuosa $\mathrm{Cu}^{3+} / \mathrm{Cu}^{2+}(2.4 \mathrm{~V})>$ $\mathrm{Co}^{3+} / \mathrm{Co}^{2+}(2.3 \mathrm{~V})>\mathrm{Ni}^{3+} / \mathrm{Ni}^{2+}(1.92 \mathrm{~V})>\mathrm{Ti}^{3+} / \mathrm{Ti}^{2+}(0.9 \mathrm{~V})$ [104].

En el caso de los sistemas $\mathrm{Li}_{2} \mathrm{Cu}_{0.5} \mathrm{M}_{0.5} \mathrm{O}_{2}$, al comparar las DOS-p obtenidas con PBE $+\mathrm{U}$ y HSE06, la conclusión general es la misma: el efecto de sustituir un átomo de $\mathrm{Cu}$ por $\mathrm{Co}, \mathrm{Ni}$ o Ti en la estructura cristalina de $\mathrm{Li}_{2} \mathrm{CuO}_{2}$, es disminuir la participación redox de los átomos de oxígeno. Al comparar las $s(\mathbf{r})$ obtenidas con SCAN, PBE, PBEsol, PBE+U y HSE06, estos últimos dos permiten obtener la misma conclusión (con cierta reserva en el caso de $\mathrm{Li}_{2} \mathrm{Cu}_{0.5} \mathrm{Ti}_{0.5} \mathrm{O}_{2}$ ): el efecto de sustituir un átomo de Cu por Co, Ni o Ti en la estructura cristalina de $\mathrm{Li}_{2} \mathrm{CuO}_{2}$, es disminuir la participación redox de los átomos de oxígeno enlazados a $\mathrm{Cu}$ y, en el caso de $\mathrm{Li}_{2} \mathrm{Cu}_{0.5} \mathrm{Ti}_{0.5} \mathrm{O}_{2}$, es posible además disminuir la participación redox también de los átomos de $O$ enlazados a Ti. Esto último también se concluye de las gráficas de la $s(\mathbf{r})$ en los sistemas $\mathrm{Li}_{2} \mathrm{Cu}_{0.75} \mathrm{M}_{0.25} \mathrm{O}_{2}$, por lo que el proceso de oxidación de este tipo de sistemas va más allá de la oxidación de los metales de transición que conforman la estructura cristalina y por lo tanto, en el diseño de nuevos materiales catódicos, el potencial redox del sitio metálico puede ser insuficiente, en casos donde los átomos de oxígeno sean los más propensos a oxidarse, haciendo que el proceso redox sea limitado por el oxígeno y no por el metal de transición.

La $s(\mathbf{r})$ es un índice de reactividad que resultó ad hoc y más práctico que la DOS-p, para el estudio redox en el contexto del proceso de carga de los materiales catódicos estudiados en este trabajo, ya que no sólo permite hacer las mismas observaciones que al analizar la DOS-p sino que, además, es lo suficientemente sensible (dependiendo del funcional de intercambio-correlación utilizado) para detectar, los átomos más susceptibles a oxidarse y con ello poder comparar directamente el efecto de un metal sustituyente, como Co, Ni o Ti en este trabajo. Ya que el alcance del trabajo llega sólo a estudiar el efecto de los ambientes químicos generados por los metales sustituyentes $\mathrm{M}$ en la estructura del $\mathrm{Li}_{2} \mathrm{CuO}_{2}$, sólo podemos sugerir que, generar ambientes químicos Ti-O en la estructura cristalina del $\mathrm{Li}_{2} \mathrm{CuO}_{2}$ (como en el caso de $\mathrm{Li}_{2} \mathrm{Cu}_{0.5} \mathrm{Ti}_{0.5} \mathrm{O}_{2}$ y Li $\mathrm{Lu}_{0.75} \mathrm{Ti}_{0.25} \mathrm{O}_{2}$ ), pueden disminuir significativamente la participación redox de $\mathrm{O}$ en el proceso de carga y con ello posiblemente disminuir la liberación de $\mathrm{O}_{2}$, lo que podría repercutir positivamente en una mejor retención de la capacidad ante ciclos repetidos de carga-descarga (mejor ciclabilidad), así mismo, también es de esperarse una disminución en el voltaje de inserción de litio. 


\subsection{Perspectivas}

Con los resultados de este trabajo se identificó que en $\mathrm{Li}_{2} \mathrm{Cu}_{0.5} \mathrm{Ti}_{0.5} \mathrm{O}_{2}$, desde la perspectiva de la Química Cuántica, los átomos de Ti y Cu son los más susceptibles a oxidarse respecto de los átomos de oxígeno. Por lo anterior, resulta importante contrastar con datos experimentales, las predicciones establecidas en este trabajo.

La blandura local puede ser utilizada como un criterio para la selección de candidatos como materiales catódicos, ya que permite identificar los átomos involucrados en el proceso de oxidación de un material catódico. Bajo la perspectiva de este trabajo, la oxidación de oxígeno es indeseable, sin embargo, en el contexto de los materiales catódicos denominados abundantes en litio (lithium-rich layered transition metal oxides), la oxidación reversible de los átomos de oxígeno es la responsable del aumento de la capacidad en dichos sistemas, por lo que es un fenómeno deseable y resulta interesante estudiar el comportamiento de la blandura local en este tipo de materiales catódicos. Sin embargo, el uso de la $s(\mathbf{r})$ no está limitado al estudio de materiales catódicos, sino que puede ser aplicado en otros fenómenos redox ajenos a las baterías de ion litio, tal como se hizo en el apéndice 9.2. Es por ello que el uso de la $s(\mathbf{r})$ en el estudio de otros procesos de oxidación puede contribuir al entendimiento de éstos, mediante un presentación visual muy amigable y práctica para su interpretación (ver por ejemplo el apéndice 9.2). 


\subsection{Definiciones de los parámetros de desempeño de las baterías} de ion litio

- Energía específica (Specific energy): Mide la cantidad de energía que puede ser almacenada y liberada por unidad de masa de la batería. Sus unidades son $\mathrm{Wh} / \mathrm{kg}$ y puede ser obtenida multiplicando la capacidad específica $(\mathrm{Ah} / \mathrm{kg})$ con el voltaje de operación de la batería (V) [3].

- Capacidad específica (Specific capacity): Mide la cantidad de carga por unidad de masa que puede ser almacenada reversiblemente $[1,3]$.

- Ciclabilidad (Cyclability): Mide la reversibilidad del proceso de inserción y extracción de ion litio, en términos del número de ciclos de carga y descarga antes de que la batería pierda significativamente energía o ya no pueda mantener su funcionalidad [3].

- Seguridad (Safety): Se refiere a prevenir o aislar fallas en el funcionamiento de la batería y no afectar el ciclo de vida de la batería [3].

- Tolerancia de abuso (Abuse tolerance): Es un requisito importante para la aplicación práctica de las BIL, especialmente en vehículos eléctricos. Comprende generalmente evaluaciones del tipo mecánico, térmico y eléctrico [3].

- Velocidad de carga/Velocidad de descarga (Rate of charge/Rate of discharge): Mide que tan rápido la batería puede ser cargada o descargada, se le conoce generalmente como C-rate [3].

- Densidad de energía (energy density): Es el producto de la capacidad específica del material que constituye al electrodo (ánodo o cátodo) y el voltaje de trabajo [1]. 
- Voltaje de trabajo (working voltage): Diferencia de potencial eléctrico entre el cátodo y ánodo [1].

\subsection{Análisis de la densidad de estados proyectada y la blandura lo- cal en minerales}

En esta sección se utilizó la DOS-p y $s(\mathbf{r})$ en un contexto diferente al proceso de oxidación en materiales catódicos para BIL. La biolixiviación (bioleaching) es un proceso de lixiviación de metales, aplicado a las menas, utilizando bacterias. Es una tecnología simple y efectiva para la extracción de metales a partir de menas de bajo grado y concentrados minerales [105]. Se ha propuesto que el papel de la bacteria en el proceso de lixiviación es el de oxidar sulfuros metálicos a sulfatos mediante reducción del oxígeno atmosférico [105-107]. El proceso de biolixiviación utilizado para la extracción de metales en minerales de sulfuro, convierte a los sulfuros metálicos insolubles en sulfatos metálicos solubles [105]. Ejemplos de minerales de sulfuro son: calcopirita, arsenopirita, pirita y marcasita, los cuales son sometidos al proceso de biolixiviación para la extracción de $\mathrm{Cu}$ (calcopirita) y Fe (arsenopirita, pirita y marcasita) con diferentes propósitos, tal y como se explicará más adelante. Se han propuesto mecanismos de oxidación para cada uno de estos sistemas y el propósito de esta sección fue comparar la información obtenida a través de la DOS-p y $s(\mathbf{r})$, con los primeros pasos de oxidación propuestos en la literatura para cada uno de estos minerales. Los cuatro sistemas fueron estudiados bajo una situación de oxidación que involucra 1 y 2 electrones. Previo a la obtención de la $D O S-p$ y $s(\mathbf{r})$ de cada sistema, se realizaron las correspondientes pruebas de convergencia en malla de puntos $k$ y energía de corte; posteriormente, se relajó cada sistema utilizando $\mathrm{PBE}+\mathrm{U}$ [60], con los $U_{\text {efectivo }}$ de la Tabla 9-1. Finalmente se obtuvieron la DOS-p y $s(\mathbf{r})$ mediante cálculos single point utilizando el funcional HSE06 [61] y los datos de la Tabla 9-2.

Tabla 9-1: $U_{\text {efectivo }}$ utilizado para cada metal de transición en calcopirita, arsenopirita, pirita y marcasita

\begin{tabular}{ccc}
\hline Metal de transición & $U_{\text {efectivo }}(\mathrm{eV})$ & Referencia \\
$\mathbf{C u}$ & 6.0 & {$[79]$} \\
$\mathbf{F e}$ & $1.5^{\mathrm{a}}$ & {$[108,109]$} \\
$\mathbf{F e}$ & $4.3^{\mathrm{b}}$ & {$[110]$} \\
\hline \hline
\end{tabular}

${ }^{\text {a }} U_{\text {efectivo }}$ utilizado para Fe en arsenopirita [109], pirita $[108,109]$ y marcasita [108].

${ }^{\mathrm{b}} U_{\text {efectivo }}$ utilizado para cada átomo de Fe coordinado a cuatro átomos de S, en calcopirita [110]. 
Tabla 9-2: Malla de puntos $k$ y energía de corte utilizadas para la obtención de la DOS-p y $s(\mathbf{r})$ de cada mineral con el funcional HSE06

\begin{tabular}{ccc}
\hline \hline Sistema & Malla de puntos $k$ & Energía de corte (eV) \\
Calcopirita & $8 \times 8 \times 4$ & 750 \\
Arsenopirita & $6 \times 6 \times 6$ & 750 \\
Pirita & $6 \times 6 \times 6$ & 700 \\
Marcasita & $8 \times 4 \times 8$ & 750 \\
\hline \hline
\end{tabular}

\subsubsection{Calcopirita}

Calcopirita $\left(\mathrm{CuFeS}_{2}\right)$ es el mineral de cobre más importante, debido a que representa aproximadamente el $70 \%$ de la reserva de cobre en el mundo [111]. El proceso de biolixiviación en este mineral presenta el problema de que sólo es posible extraer alrededor del $50 \%$ del $\mathrm{Cu}$, debido a reacciones de pasivación que causan que la velocidad de lixiviación (oxidación) sea lenta. Se han propuesto diferentes reacciones de oxidación, las cuales llevan a la generación de diferentes sistemas azufrados. Una de estas reacciones es la siguiente, en medio ácido [112,113] y con la bacteria Acidithiobacillus ferrooxidans:

$$
\mathrm{CuFeS} \mathrm{S}_{2(s)} \longleftrightarrow \mathrm{Cu}_{1-x} \mathrm{Fe} \mathrm{S}_{2(s)}+x C u_{(l)}^{2+}+(2 x) e^{-}
$$

en la cual, el $\mathrm{Cu}^{1+}$ (en la calcopirita [114]) es liberado y oxidado a $\mathrm{Cu}^{2+}$ durante la reacción $[112,113]$. Con este antecedente, se planteó obtener la DOS-p y $s(\mathbf{r})$ de calcopirita $\left(\mathrm{CuFeS}_{2}\right)$ e identificar los estados electrónicos más susceptibles a ser oxidados, considerando la sustracción de uno así como de dos electrones y comparar con esta reacción.

En la Figura 9-1 se muestra la DOS-p obtenida con el funcional HSE06, en la cual se puede apreciar que los estados electrónicos involucrados en el proceso de oxidación (tanto de uno como de dos electrones), corresponden en primer lugar a $\mathrm{Cu}$, en segundo lugar los estados de $\mathrm{S}$ y finalmente los estados electrónicos de Fe. Por otro lado, en la Tabla 9-3 se muestra la $s(\mathbf{r})$ obtenida con HSE06, donde se puede observar que las regiones susceptibles a la pérdida de un electrón, corresponden a sitios ubicados en los átomos de $\mathrm{Cu}$, mientras que la pérdida de dos electrones involucra, en su mayoría, regiones sobre los $\mathrm{Cu}$ y en menor medida, regiones sobre los S. Cabe mencionar que no se observó la participación de los átomos de Fe en el proceso de oxidación. A partir de la información obtenida tanto de DOS- $p$ como de $s(\mathbf{r})$, se puede proponer que el proceso de pérdida de uno y dos electrones involucra, principalmente, a los estados electrónicos correspondientes a $\mathrm{Cu}$, con participación secundaria de los estados de $S$, lo cual corresponde, en parte, a la reacción 9-1. Además, al igual que en casos anteriores, la misma información proporcionan la DOS-p y la $s(\mathbf{r})$, sin embargo, el proceso de obtención de la $s(\mathbf{r})$ es más práctico y visual, permitiendo obtener una imagen clara de los sitios reactivos en el sistemas y recordando que, dada la condición en la ecuación 6-7, las $s(\mathbf{r})$ pueden ser comparadas directamente, entre sistemas. 


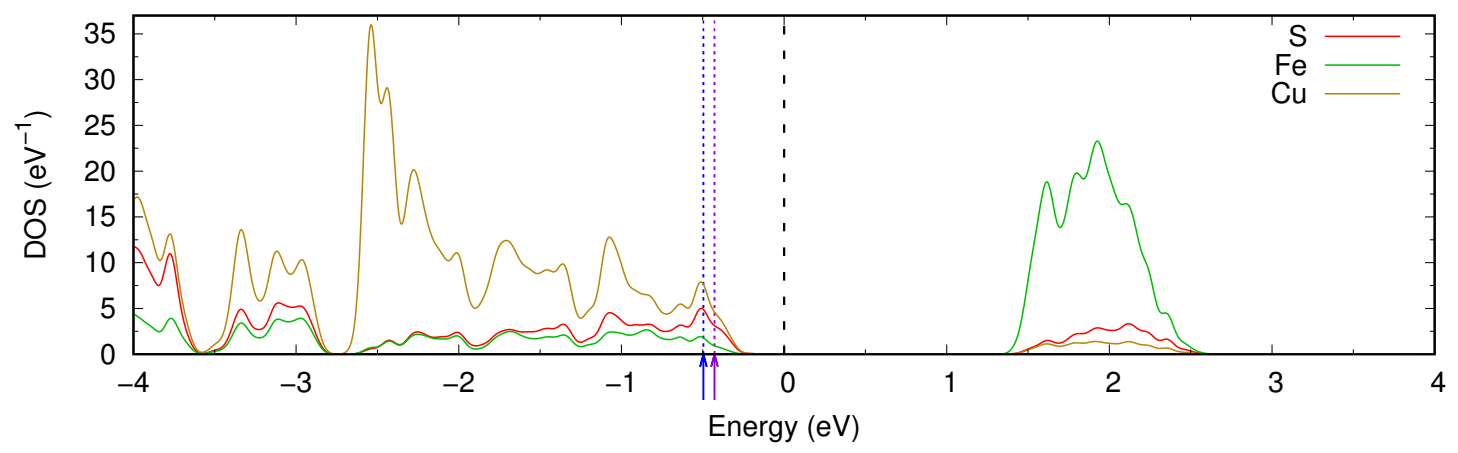

Figura 9-1: DOS-p de calcopirita, obtenida con HSE06. La línea punteada de color negro representa el nivel de Fermi, las dos líneas punteadas paralelas representan el intervalo de energía respecto al nivel de Fermi donde el sistema puede perder 1 (violeta) o 2 (azul) electrones. 
Tabla 9-3: Celda unitaria de $\mathrm{CuFeS}_{2}$ y blandura local obtenida con HSE06, para un proceso de pérdida de 1 y 2 electrones
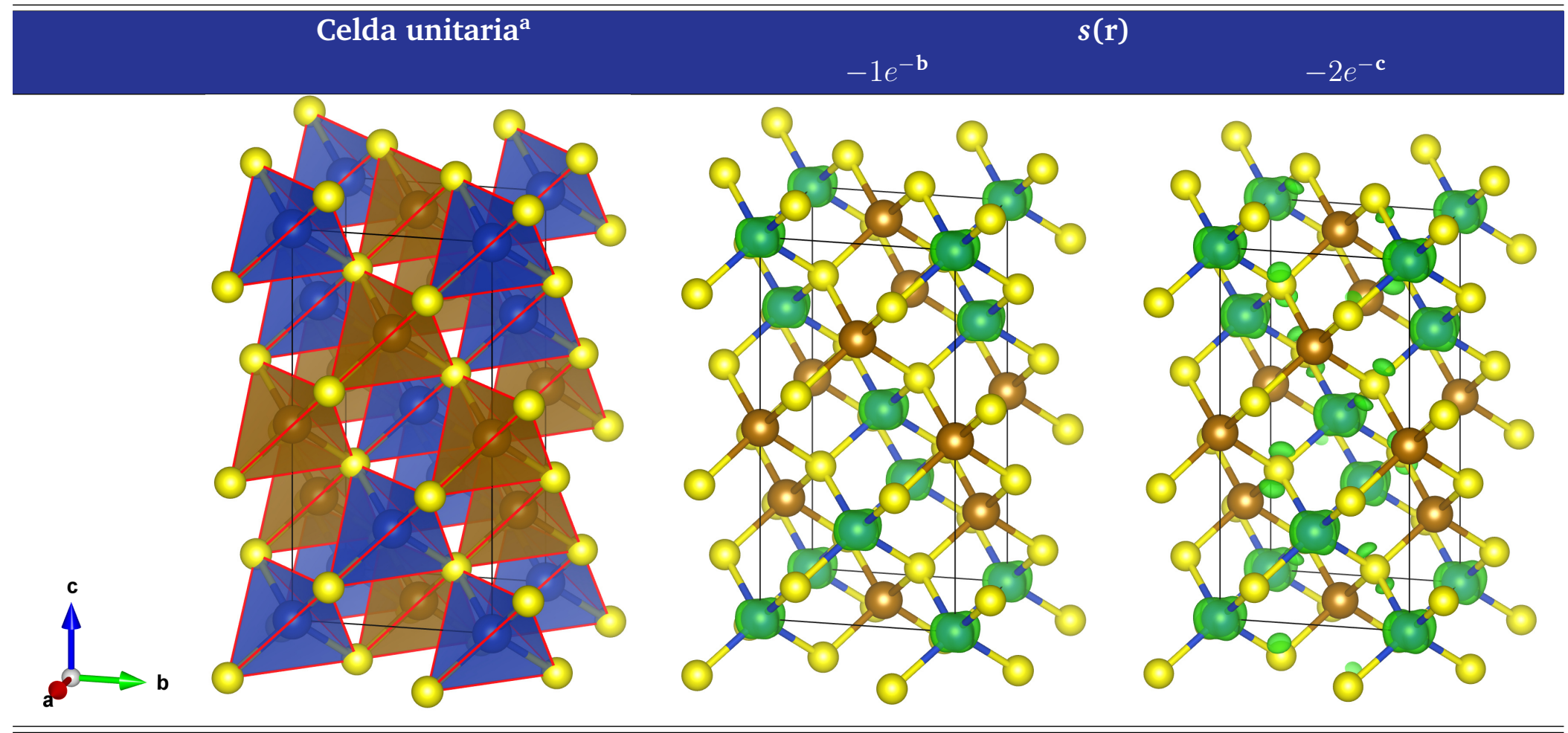

" Los átomos de $\mathrm{Cu}, \mathrm{Fe}$ y S son representados con esferas de color azul, café y amarillo, respectivamente.

a La celda unitaria es encerrada en negro mientras que los poliedros de coordinación son delimitados en color rojo.

b Gráfica de la blandura local (isovalor de 0.245 unidades atómicas) cuando el sistema experimenta un proceso de sustracción de un electrón.

c Gráfica de la blandura local (isovalor de 0.245 unidades atómicas) cuando el sistema experimenta un proceso de sustracción de dos electrones. 


\subsubsection{Arsenopirita}

Dentro de las actividades mineras, la extracción de metales libera al medio ambiente cantidades significantes de arsenopirita (FeAsS) y otros minerales de azufre que contienen elementos tóxicos tales como $\mathrm{As}, \mathrm{Pb}, \mathrm{Cr}$ y Cd [115]. La arsenopirita es la principal fuente mineral de As en la litósfera [116] y parte de su estudio ha sido dirigido a comprender:

- El mecanismo de liberación de As al ambiente mediante el desgaste (oxidación) de arsenopirita, con el propósito de encontrar soluciones para reducir el riesgo de contaminación y poder proteger la salud humana así como la integridad del medio ambiente [115].

- Su oxidación en los procesos de biorremediación y biolixiviación, donde se utilizan microorganismos que oxidan Fe y/o As, por ejemplo, Acidithiobacillus thiooxidans. El conocimiento de la interacción entre arsenopirita y el microorganismo oxidante (p. ej. A. thiooxidans) puede ayudar a controlar la reactividad de arsenopirita y poder diseñar procesos de biolixiviación que eviten la liberación de As al ambiente, el cual resulta ser tóxico [116].

Lara y colaboradores [115] estudiaron la oxidación de arsenopirita bajo condiciones de suelo calcáreo simulado y concluyeron que en el paso inicial de oxidación de arsenopirita, se forma un compuesto tipo $\mathrm{Fe}_{1-x} \mathrm{As}_{1-y} \mathrm{~S}$ en conjunto con As soluble, $\mathrm{S}$ y especies ferrosas, esto sugiere que la oxidación de arsenopirita involucra la oxidación de Fe como de As, por lo que resulta de interés conocer desde el punto de vista teórico, los estados electrónicos más susceptibles a ser oxidados, todo esto mediante la $D O S-p$ así como la $s(\mathbf{r})$ y comparar con lo reportado por estudios experimentales. En la Figura 9-2 se ilustra la DOS- $p$ de

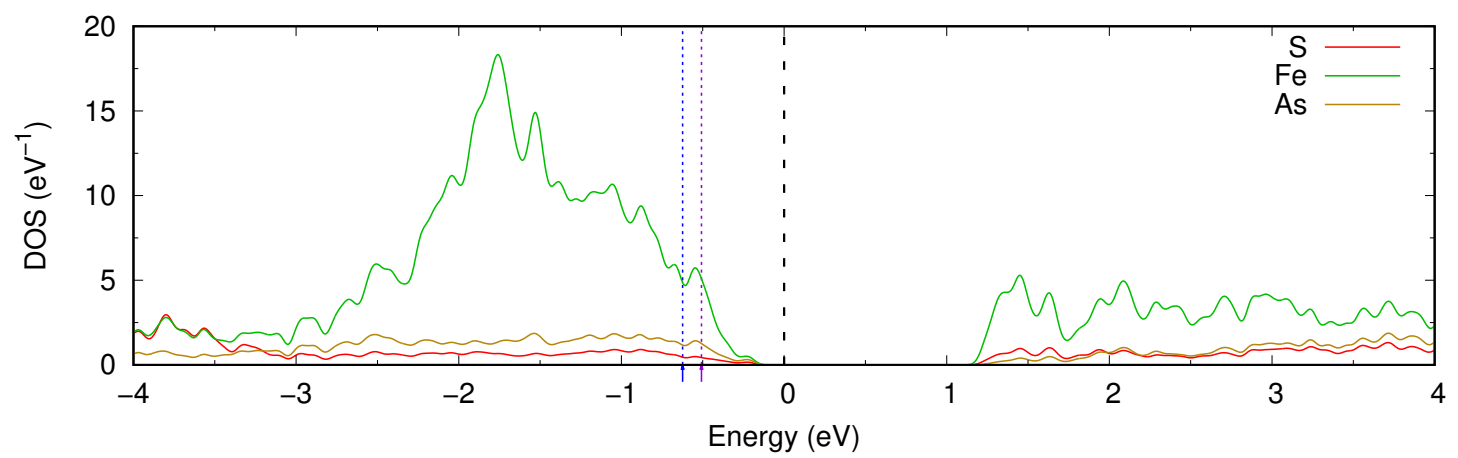

Figura 9-2: DOS-p de arsenopirita, obtenida con HSE06. La línea punteada de color negro representa el nivel de Fermi, las dos líneas punteadas paralelas representan el intervalo de energía respecto al nivel de Fermi donde el sistema puede perder 1 (violeta) o 2 (azul) electrones. 
FeAsS obtenida con HSE06, en la cual, se indican los intervalos sobre los cuales se pierde uno y dos electrones. Tal y como se muestra en la DOS-p de este sistema, los estados más susceptibles a ser oxidados son los correspondientes a Fe, en segundo lugar los de As y por último los de S. En la Tabla 9-4, se muestra la $s(\mathbf{r})$ obtenida con HSE06, se puede visualizar que las regiones susceptibles a la pérdida de uno y dos electrones corresponden a los átomos de Fe (mayoritariamente) así como la región de enlace Fe-As (en menor proporción). A partir de estos resultados se puede proponer que: 1) en el proceso de oxidación, a partir de la DOS-p, tanto los átomos de Fe como lo de As son propensos a ser oxidados, sin embargo 2) al observar la $s(\mathbf{r})$, son los átomos de Fe y la densidad electrónica en los enlaces Fe-As los que son susceptibles a la oxidación, lo cual puede explicar la liberación de As durante un proceso de oxidación en general (ya sea la oxidación natural de la arsenopirita presente en los suelos, como aquella a través de biolixiviación). Nuevamente la $s(\mathbf{r})$ proporciona la misma información que DOS-p, con la ventaja de que la $s(\mathbf{r})$ muestra que antes de comprometerse la oxidación directa de As, es el enlace Fe-As el que participa en el proceso de oxidación. 
Tabla 9-4: Celda unitaria de FeAsS y blandura local obtenida con HSE06, para un proceso de pérdida de 1 y 2 electrones

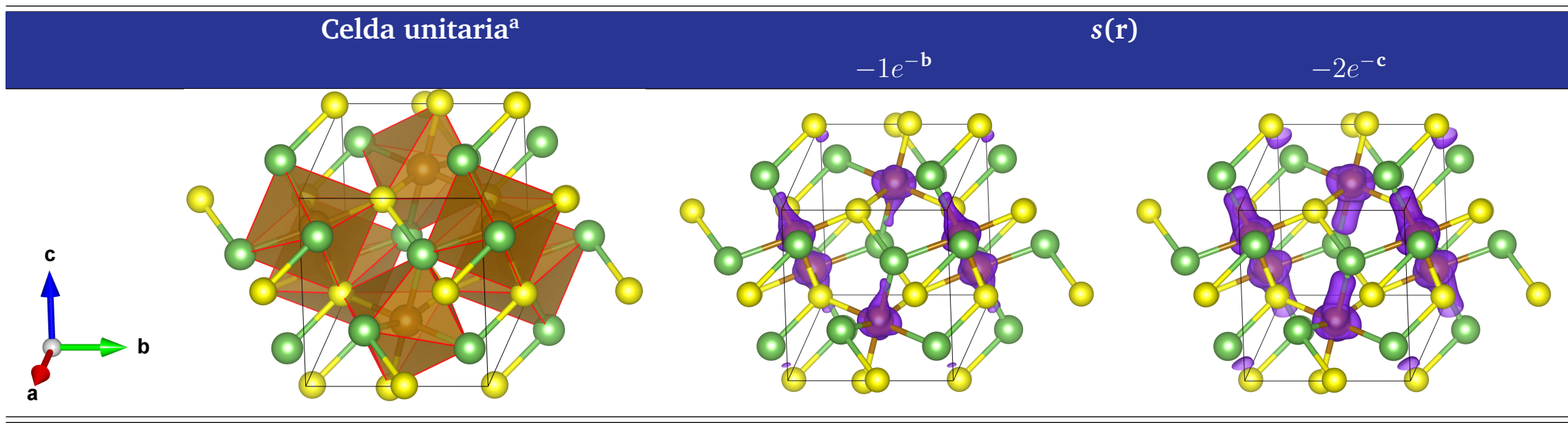

* Los átomos de Fe, As y S son representados con esferas de color café, verde y amarillo respectivamente.

${ }^{\text {a }}$ La celda unitaria es encerrada en negro mientras que los poliedros de coordinación son delimitados en color rojo.

b Gráfica de la blandura local (isovalor de 0.245 unidades atómicas) cuando el sistema experimenta un proceso de sustracción de un electrón.

c Gráfica de la blandura local (isovalor de 0.245 unidades atómicas) cuando el sistema experimenta un proceso de sustracción de dos electrones. 


\subsubsection{Pirita}

Pirita $\left(\mathrm{FeS}_{2}\right)$ es el sulfuro metálico más abundante sobre la superficie de la tierra [117]. Su proceso de desgaste ocasiona el denominado Drenaje Ácido de Roca (acid rock drainage, ARD), el cual libera elementos potencialmente nocivos ${ }^{\mathrm{a}}$ al suelo y aguas [118-120]. Respecto a la oxidación de pirita mediante el proceso de biolixiviación, se han propuesto diferentes reacciones de oxidación, una de ellas implica, en primer lugar, la oxidación de los átomos de S para formar ácido sulfúrico [105]:

$$
4 \mathrm{FeS}_{2}+14 \mathrm{O}_{2}+4 \mathrm{H}_{2} \mathrm{O} \stackrel{\text { bacteria }}{\longleftrightarrow} 4 \mathrm{FeSO}_{4}+4 \mathrm{H}_{2} \mathrm{SO}_{4}
$$

para después oxidar a sulfato de hierro (III) [105]:

$$
4 \mathrm{FeSO}_{4}+\mathrm{O}_{2}+2 \mathrm{H}_{2} \mathrm{SO}_{4} \stackrel{\text { bacteria }}{\longleftrightarrow} 2 \mathrm{Fe}_{2}\left(\mathrm{SO}_{4}\right)_{3}+2 \mathrm{H}_{2} \mathrm{O}
$$

Por otro lado, se ha propuesto que el primer paso en la oxidación de pirita, asistido por bacterias, involucra la liberación de electrones y de iones $\mathrm{Fe}^{2+}$ [113]:

$$
F e S_{2(s)} \longleftrightarrow F e_{1-x} S_{2(2)}+x F e_{l}^{2+}+(2 x) e^{-}
$$

los cuales posteriormente se oxidan a $\mathrm{Fe}^{3+}$ [113]:

$$
\mathrm{Fe}^{2+} \longleftrightarrow \mathrm{Fe}^{3+}+1 e^{-}
$$

A partir de estas dos propuestas de oxidación de pirita, se decidió estudiar, desde la perspectiva de la Mecánica Cuántica, el proceso inicial de oxidación de este sistema por medio del análisis de la DOS- $p$ y la $s(\mathbf{r})$ y comparar con estas reacciones de oxidación.

En la Figura 9-3 se ilustra la DOS-p de pirita, obtenida con HSE06, donde claramente se aprecia que los estados electrónicos más susceptibles a ser oxidados ${ }^{\mathrm{b}}$, son en su mayoría, pertenecientes a los átomos de Fe. Por otro lado, en la Tabla 9-5 se muestra la $s(\mathbf{r})$, en la cual se aprecia que las regiones susceptibles a la pérdida de un electrón, corresponden a los átomos de Fe del sistema. En el caso de la sustracción de dos electrones, las regiones situadas en los átomos de Fe son las más susceptibles, con muy poca participación de los estados electrónicos correspondientes a los átomos de $\mathrm{S}$. Con esos resultados se establece que, desde la perspectiva teórica, el proceso de oxidación de pirita es, inicialmente, por medio de la oxidación de los átomos de Fe del sistema, lo cual apoya las reacciones 9-4 y 9-5.

En un estudio realizado por Lara y colaboradores [113], se reportó que pirita durante la oxidación, libera más carga que calcopirita, siendo pirita más activa que calcopirita. Para abordar esto desde la perspectiva teórica, consideremos la siguiente observación.

\footnotetext{
aPor ejemplo, As, Pb, Cr, Hg [118-120].

bTanto a perder un electrón como a perder dos electrones.
} 


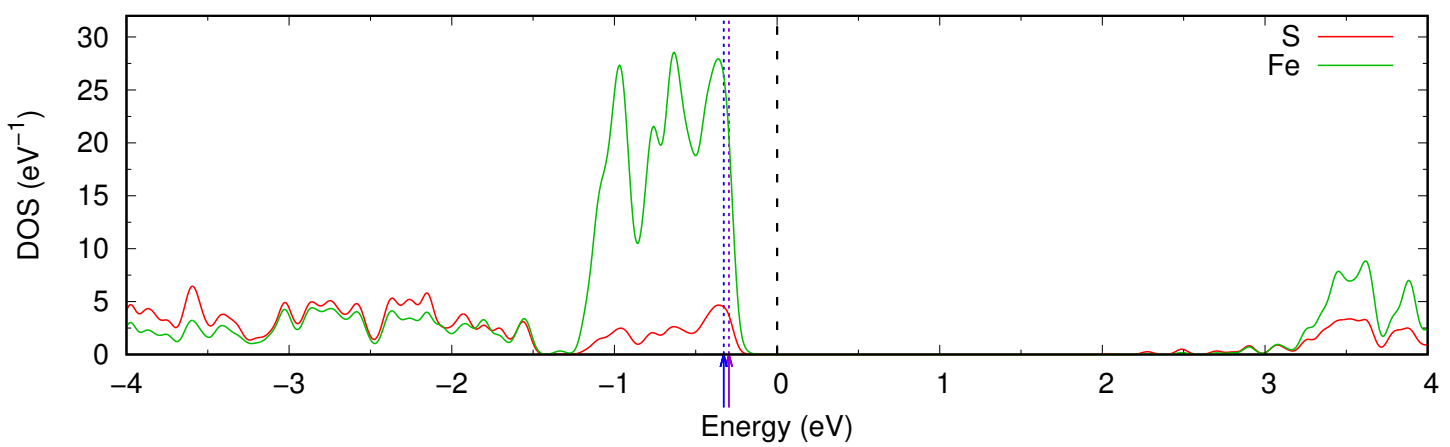

Figura 9-3: DOS-p de pirita, obtenida con HSE06. La línea punteada de color negro representa el nivel de Fermi, las dos líneas punteadas paralelas representan el intervalo de energía respecto al nivel de Fermi donde el sistema puede perder 1 (violeta) o 2 (azul) electrones.

De acuerdo a la DOS- $p$ de cada sistema, tomando como referencia el nivel de Fermi en cada uno, al moverse hacia la izquierda (hasta $1 \mathrm{eV}$ ) de éste (en cada sistema), es decir hacia estados electrónicos ocupados más internos, la integral de la DOS es la cuarta parte del número de electrones totales de su celda unitaria, en el caso de pirita, mientras que en el caso de calcopirita, la integral de la DOS es, aproximadamente la catorceava parte del número de electrones totales de su celda unitaria. Por lo anterior, existen más electrones que pueden ser sustraídos en la pirita que en el caso de calcopirita, en ese intervalo de energía, por ello, es de esperarse que la oxidación de pirita libere más carga que calcopirita. Hasta el momento se puede establecer que, tomando como referencia los resultados de pirita, el efecto de incluir $\mathrm{Cu}$ o As en la estructura de la pirita (calcopirita y arsenopirita, respectivamente), no sólo tiene un impacto en la estructura cristalina del sistema, sino además en la distribución de los estados susceptibles a ser oxidados, siendo diferente en cada uno de estos minerales. Estas distribuciones diferente de estados, han podido obtenerse mediante la $P D O S$ y la $s(\mathbf{r})$ de cada sistema, por lo que resultó interesante conocer la DOS- $p$ así como la $s(\mathbf{r})$ en dos sistemas que se diferencian únicamente en la distribución de los poliedros de coordinación, lo cual se discute en la siguiente sección. 
Tabla 9-5: Celda unitaria de $\mathrm{FeS}_{2}$ (pirita) y blandura local obtenida con HSE06, para un proceso de pérdida de 1 y 2 electrones

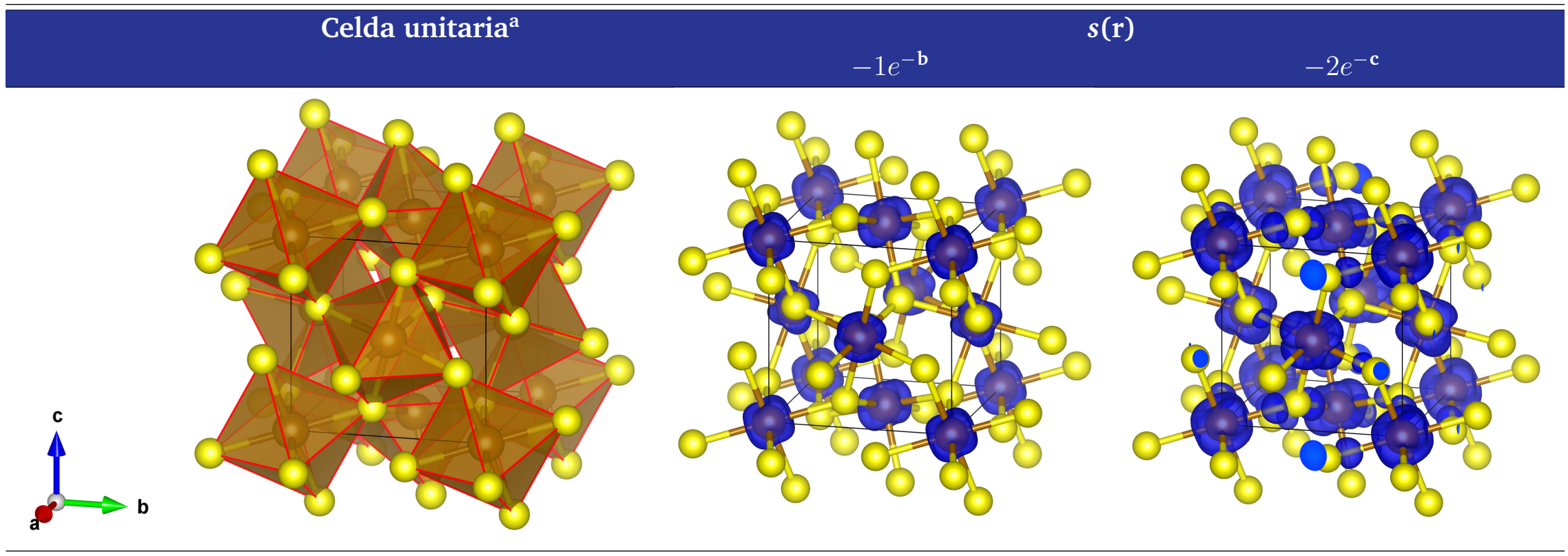

\footnotetext{
* Los átomos de Fe y S son representados con esferas de color café y amarillo respectivamente.

a La celda unitaria es encerrada en negro mientras que los poliedros de coordinación son delimitados en color rojo.

b Gráfica de la blandura local (isovalor de 0.245 unidades atómicas) cuando el sistema experimenta un proceso de sustracción de un electrón.

c Gráfica de la blandura local (isovalor de 0.245 unidades atómicas) cuando el sistema experimenta un proceso de sustracción de dos electrones.
} 


\subsubsection{Marcasita}

Marcasita y pirita $\left(\mathrm{FeS}_{2}\right)$ son dos minerales abundantes en varias menas y depósitos de carbón. El desgaste de estos minerales forma ácido sulfúrico e ion férrico $\left(\mathrm{Fe}^{3+}\right)$, los dos principales constituyentes del ARD [121], que es un problema ambiental serio en la industria minera [122] que ocasiona contaminación severa de aguas y suelos [123].

Marcasita y pirita son isoquímicos [121], es decir poseen la misma composición, pero con estructura cristalina diferente. En pirita, cada átomo de Fe está coordinado a seis átomos de azufre formando octaedros, los cuales, comparten vértice [108] tal y como se muestra en la Tabla 9-5. En marcasita, también cada átomo de Fe está coordinado a seis átomos de azufre formando octaedros, sin embargo, estos octaedros comparten borde en dirección del eje $z$, mientras que en las otras direcciones comparten vértice [124] (Tabla 9-6). Dada las características anteriores, el propósito de esta sección fue conocer el impacto de la diferente orientación de los octaedros, en marcasita respecto a pirita, tanto en la DOS- $p$ como en la $s(\mathbf{r})$ y por tanto en el proceso de oxidación.

En la Figura 9-4 se presenta la DOS-p de marcasita obtenida con HSE06, en la cual, al

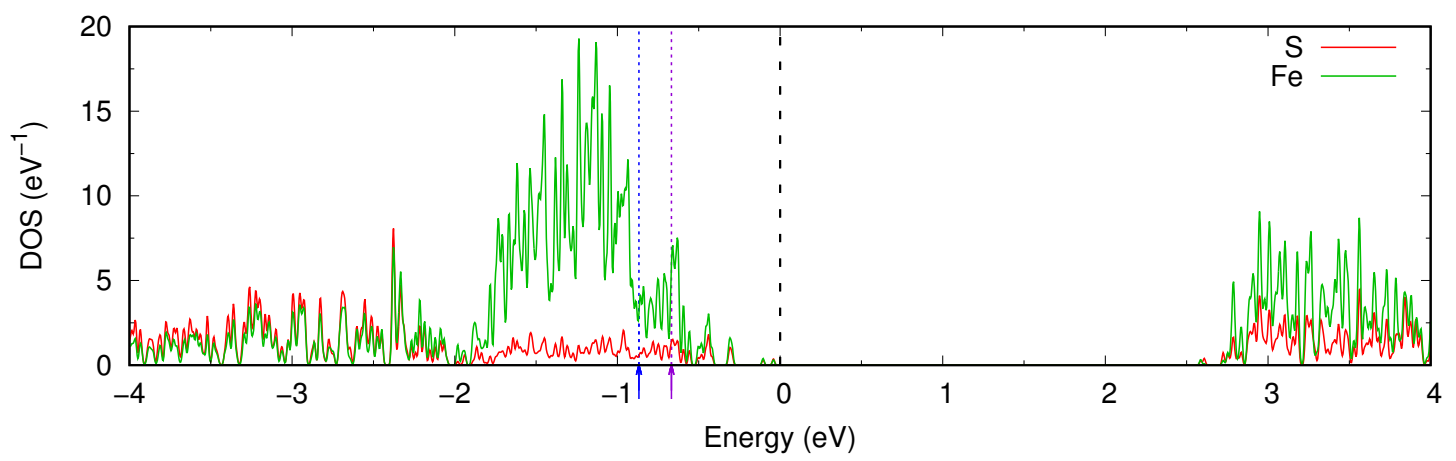

Figura 9-4: DOS-p de marcasita, obtenida con HSE06. La línea punteada de color negro representa el nivel de Fermi, las dos líneas punteadas paralelas representan el intervalo de energía respecto al nivel de Fermi donde el sistema puede perder 1 (violeta) o 2 (azul) electrones.

igual que la DOS-p de pirita, los estados mayoritarios, en la región de pérdida de uno y dos electrones, corresponden a Fe, mientras que los correspondientes a $\mathrm{S}$ son minoritarios. $\mathrm{Al}$ observar las $s(\mathbf{r})$ de la Tabla 9-6, se pude notar que existe una diferencia sutil entre estas $s(\mathbf{r})$ y las de pirita. En el caso de pirita, se mencionó que la participación de los átomos de azufre en el proceso de oxidación es notable solo ante la pérdida de dos electrones, sin embargo, en el caso de marcasita, la participación de los átomos de $\mathrm{S}$ se observa tanto en el caso de la sustracción de uno como en el de dos electrones. Con este resultado se establece que existen cambios en la DOS-p y $s(\mathbf{r})$ debido a una diferente orientación de los octaedros en el caso de marcasita con respecto a la orientación de los octaedros en pirita, 
sin embargo, los átomos de Fe siguen siendo los más susceptibles a ser oxidados.

Respecto a los minerales estudiados en la sección 9.2, se concluye que el análisis de la DOS- $p$ y la $s(\mathbf{r})$ en cada sistema, sustenta las reacciones de oxidación propuestas para cada uno, enriqueciendo el conocimiento el proceso de oxidación que sucede en cada sistema, siendo la $s(\mathbf{r})$ una herramienta visual práctica. En el caso de calcopirita, los átomos de $\mathrm{Cu}$ de la red cristalina del sistema, son los más susceptibles a ser oxidados, mientras que en caso de arsenopirita, los átomos de Fe son las más propensos a ser oxidados y en segundo lugar, el enlace Fe-As, lo cual debe evitarse para no liberar As al ambiente. Respecto a pirita y marcasita, también los átomos de Fe son las más propensos a ser oxidados y existen diferencias entre sus DOS- $p$ y $s(\mathbf{r})$ por lo que se concluye que tanto DOS- $p$ como $s(\mathbf{r})$ son sensibles al ambiente químico que rodea a cada átomo del sistema y por lo tanto son adecuados para obtener información del proceso de oxidación y ayudar a la generación de soluciones a los diferentes problemas planteados en este contexto. Esto permite concluir que la $s(\mathbf{r})$ puede ser utilizada en el estudio de procesos de oxidación más allá de lo referente a los materiales catódicos para las BIL. 
Tabla 9-6: Celda unitaria de $\mathrm{FeS}_{2}$ (marcasita) y blandura local obtenida con HSE06, para un proceso de pérdida de 1 y 2 electrones

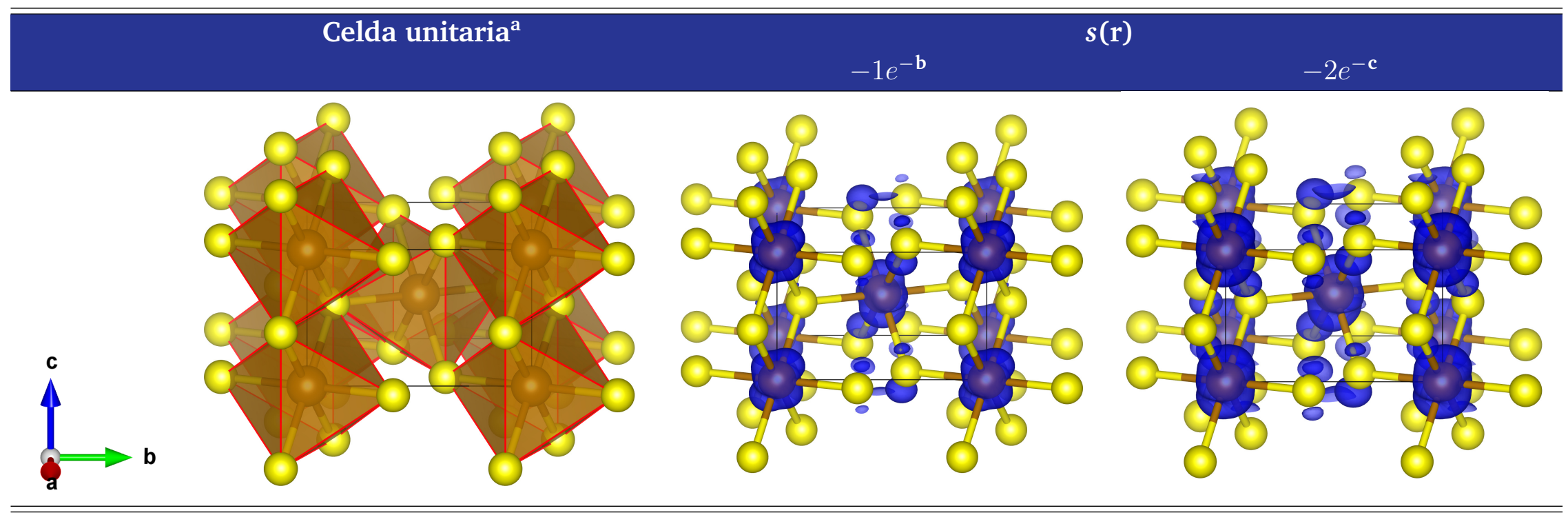

\footnotetext{
* Los átomos de Fe y S son representados con esferas de color café y amarillo respectivamente.

a La celda unitaria es encerrada en negro mientras que los poliedros de coordinación son delimitados en color rojo.

${ }^{\mathrm{b}}$ Gráfica de la blandura local (isovalor de 0.245 unidades atómicas) cuando el sistema experimenta un proceso de sustracción de un electrón.

c Gráfica de la blandura local (isovalor de 0.245 unidades atómicas) cuando el sistema experimenta un proceso de sustracción de dos electrones.
} 


\subsection{Artículo}

En esta sección se inserta el artículo publicado, derivado de este trabajo. 


\title{
Using local softness to reveal oxygen participation in redox processes in cathode materials
}

\author{
Luis Ignacio Perea-Ramírez ${ }^{1}$. Alfredo Guevara-García ${ }^{2}$. Marcelo Galván ${ }^{1}$
}

Received: 19 April 2018 / Accepted: 20 July 2018

(C) Springer-Verlag GmbH Germany, part of Springer Nature 2018

\begin{abstract}
In this paper, the use of chemical local softness $\mathrm{s}(\mathbf{r})$ is proposed as an alternative way of analyzing the initial redox processes that occur in cathode materials used for lithium-ion batteries. It is shown that the chemical local softness is a quantity able to capture the same effects as the standard analysis based on the projected density of states. Because of its own nature, the local softness reveals the atomic sites involved in charge-transfer events and allows a quantitative comparative analysis between different materials. As pointed out by Johannes et al. (Solid State Ion 286:83-89, 2016), this analysis can be used as an indicator of stability of cathode materials for Li-ion batteries.
\end{abstract}

Keywords Local softness · Cathode materials $\cdot$ Lithium-ion batteries

\section{Introduction}

The importance of the participation of the oxygen atoms in the redox processes that occur in transition metal oxides used as cathode materials in lithium-ion batteries has been pointed out since early studies [1] and still is an active research field $[2,3]$. The oxygen redox properties can increase or decrease the overall performance of a specific cathode material. For example, in lithium-rich layered transition metal oxides, the oxygen oxidation can increase the capacity of the material compared to theoretical one, calculated considering that only the transition metal atoms participate in the redox process [4-6]. The last observation has been explained based on a mechanism that considers that neighboring oxygen atoms can be oxidized

This paper belongs to Topical Collection International Conference on Systems and Processes in Physics, Chemistry and Biology (ICSPPCB-2018) in honor of Professor Pratim K. Chattaraj on his sixtieth birthday

$\triangle$ Marcelo Galván

mgalvan@xanum.uam.mx

1 Departamento de Química, Universidad Autónoma Metropolitana-Iztapalapa, Apartado Postal, 55-534, 09340, Iztapalapa, D.F., México

2 CONACYT-Universidad Autónoma Metropolitana-Iztapalapa, Departamento de Química, Apartado Postal, 55-534, 09340, Iztapalapa, D.F., México
[2] producing "oxygenated species" of the general formula $\mathrm{O}_{2}^{n-}$, which with a certain degree of reversibility, can be oxidized or reduced in the charge/discharge process $[4,7,8]$ and lead to oxygen evolution [5, 7, 9-11]. Nevertheless, it looks like that the generation of these $\mathrm{O}_{2}^{n-}$ species depends on specific conditions [2] and sometimes a migration of the transition metal is involved during this process [10,12-15]. It seems that a universal redox mechanism for these systems does not exist.

On the other hand, for laminar transition metal oxides, a diminution in security and capacity have been related to oxygen evolution $[16,17]$. This phenomenon has a significant impact in overload or overheating situations in such materials. The evolution of $\mathrm{O}_{2}$ has been attributed to the nonreversible oxidation of the anionic oxygen sublattice $\left(\mathrm{O}^{2-}\right)$ through the formation of peroxide [18]:

$$
\begin{aligned}
& 2 \mathrm{O}^{2-} \rightarrow \mathrm{O}_{2}^{2-}+2 e^{-} \\
& \mathrm{O}_{2}^{2-} \rightarrow \mathrm{O}_{2}+2 e^{-}
\end{aligned}
$$

Recently, Johannes et al. [18] showed that the oxygen character in the density of states (DOS) can be used as an indicator of the stability of Li-ion battery cathode materials. Basically, they proposed that by analyzing the contribution of the projected-DOS of each type of atom to the total DOS near the Fermi level, information of the atoms involved in the redox processes that occur in cathode materials 
can be extracted. The analysis is based on the idea that if the oxygen character surpasses, or competes with, the contribution of the transition metal in the edge of the valence band, then during the process of extracting electrons from the material, the system would have a tendency towards the oxidation of $\mathrm{O}^{2-}$ and consequently release $\mathrm{O}_{2}$. In this work, we proposed an alternative way of analyzing this phenomenon by using the local softness [19], which results in a quantitative visual way to understand the initial redox reactions in this type of materials. We first present a small review of the local softness and how it is computed, then we apply it to some well known materials $\left(\mathrm{LiFePO}_{4}\right.$ and $\mathrm{LiCoO}_{2}$ ) and analyze the effect related to the substitution of the transition metal center in $\mathrm{Li}_{2} \mathrm{CuO}_{2}$.

\section{Local softness}

Local softness, $s(\mathbf{r})$, is a response coefficient defined within the formalism of the grand canonical potential of density functional theory. Originally introduced by Yang and Parr [19], its definition for solids was extended by Cohen et al. [20]; this concept has been useful to rationalize the chemical reactivity related to electron transfer in molecules [21], solids, and surfaces [22-25]. Its definition is

$s(\mathbf{r}) \equiv\left(\frac{\partial \rho(\mathbf{r})}{\partial \mu}\right)_{v(\mathbf{r})}$

where $\rho(\mathbf{r})$ is the electron density, $\mu$ is the chemical potential and $v(\mathbf{r})$ represents the external potential. In a solid, the electron density is given in terms of the local density of states, $g(\mathbf{r}, \epsilon, \mu)$, as

$\rho(\mathbf{r}, \mu)=\int^{\mu} g(\mathbf{r}, \varepsilon, \mu) d \varepsilon$

In the expression above, following Cohen et al. [20], the explicit dependence of the local density of states on the chemical potential is emphasized. It is important to keep in mind that, for a gapless system, the chemical potential is equal to the Fermi level, $\varepsilon_{f}$, whereas for systems with a finite gap the chemical potential is in a place between the edges of the valence and conduction bands. A finite change in the density in terms of a change in the chemical potential can be expressed as,

$\rho\left(\mathbf{r}, \mu_{0}+\Delta \mu\right)=\int^{\mu_{0}+\Delta \mu} g\left(\mathbf{r}, \varepsilon, \mu_{0}+\Delta \mu\right) d \varepsilon$

In this expression, $\mu_{0}$ is a reference chemical potential. By expanding $g(\mathbf{r}, \varepsilon, \mu)$ in a Taylor series up to first order in $\mu$, around the reference chemical potential, one may obtain an expression for the density difference up to first order in the form:

$$
\begin{aligned}
& \rho\left(\mathbf{r}, \mu_{0}+\Delta \mu\right)-\rho\left(\mathbf{r}, \mu_{0}\right) \approx\left[\frac{1}{\Delta \mu} \int_{\mu_{0}}^{\mu_{0}+\Delta \mu} g\left(\mathbf{r}, \varepsilon, \mu_{0}\right) d \varepsilon\right. \\
& \left.+\int^{\mu_{0}+\Delta \mu}\left(\frac{\partial g(\mathbf{r}, \varepsilon, \mu)}{\partial \mu}\right)_{v(\mathbf{r}), \mu=\mu_{0}} d \varepsilon\right] \Delta \mu
\end{aligned}
$$

The quantity between square brackets in the equation above is the first derivative of the electron density with respect to the chemical potential in a finite differences approximation,

$$
\begin{aligned}
& s(\mathbf{r}) \approx \frac{1}{\Delta \mu} \int_{\mu_{0}}^{\mu_{0}+\Delta \mu} g\left(\mathbf{r}, \varepsilon, \mu_{0}\right) d \varepsilon \\
& +\int^{\mu_{0}+\Delta \mu}\left(\frac{\partial g(\mathbf{r}, \varepsilon, \mu)}{\partial \mu}\right)_{v(\mathbf{r}), \mu=\mu_{0}} d \varepsilon
\end{aligned}
$$

The sign of $\Delta \mu$ determines when one is dealing with accepting or donating electrons. The second term in the equation above represents the relaxation of the local density of states when the chemical potential is changed. If this relaxation is neglected, we obtain the local softness within the rigid band approximation [25]:

$s(\mathbf{r}) \approx \frac{1}{\Delta \mu} \int_{\mu_{0}}^{\mu_{0}+\Delta \mu} g\left(\mathbf{r}, \varepsilon, \mu_{0}\right) d \varepsilon$

This approximation has been able to capture the different reactivity behavior between bulk and surface oxygen atoms in alkaline earth oxides surfaces [26]. There have been some efforts to go beyond this approximation [20, 24], however in this work we applied Eq. 8 to estimate local softness. To fix the $\Delta \mu$, the condition

$\int_{\Omega} \int_{\varepsilon_{f}}^{\varepsilon_{f}+\Delta \mu} g\left(\mathbf{r}, \varepsilon, \mu_{0}\right) d \varepsilon d \mathbf{r}=1.0$

is applied, and because we are considering the oxidation of the solid the sign of $\Delta \mu$ is negative. The integral over $\mathbf{r}$ is done in the unit cell volume, $\Omega$. This condition corresponds, within this finite differences scheme, to the idea of considering the transfer of one electron. A similar condition has been applied in other contexts [2]. As mentioned by one of the reviewers, Eq. 9 will also fix the number of states below the Fermi level that contribute to the chemical response. Pinos-Rios and coworkers [27] have shown, using a topological analysis of an orbitalweighted Fukui function, that in some cases the number of states considered to be involved in the chemical response is relevant to explain the observed reactivity. Recently, Cárdenas et al. proposed a model for computing the local softness that weighs the contribution of each state in Eq. 8 "by the inverse of the square root of the negative of the energy of the orbital" [28]. Their model performs better than 
Table $1 \mathrm{U}_{\text {eff }}$ values used for the transition metals in this work

\begin{tabular}{lll}
\hline $\mathrm{TM}$ & $\mathrm{U}_{\text {eff }}(\mathrm{eV})$ & Ref. \\
\hline $\mathrm{Fe}$ & 3.71 & {$[46]$} \\
$\mathrm{Cu}$ & 6.00 & {$[47]$} \\
$\mathrm{Co}$ & 5.10 & {$[48]$} \\
$\mathrm{Ni}$ & 5.96 & {$[49]$} \\
\hline
\end{tabular}

the model derived from Eq. 8 for explaining the reactivity of carbon nanotubes.

\section{Computational details}

All the calculations were done by solving the Kohn-Sham equations under periodic conditions using the following approximations as implemented in the VASP program [29]: (1) to treat the core valence interactions the PAW method was used [30, 31]; (2) the approximations to the exchange and correlation functional used were $\mathrm{PBE}+\mathrm{U}$ in the Dudarev realization [32] for the geometry optimization(see Table 1 for the parameters used), and for the evaluation of the DOS and the local softness, the HSE06 approach [33] was used. This specific combination of exchangecorrelation approximations was used in order to save computational resources as it has been shown that some electronic properties, such as DOS, are better described using the HSE06 functional than the PBE+U approach [34]; 3 ) the truncation of the plane wave expansion was set by an energy cutoff of $900 \mathrm{eV}$ except for $\mathrm{LiFePO}_{4}$ and $\mathrm{LiCoO}_{2}$ where values of 800 and $700 \mathrm{eV}$ were used, respectively; (4) the k points sampling was of $12 \times 12 \times 4$, except for $\mathrm{LiFePO}_{4}$ and $\mathrm{LiCuO}_{2}$ where a $4 \times 6 \times 6$ and $6 \times 12 \times 6$ sampling were used, respectively. Worth mentioning, the lowest energy magnetization state was determined for each

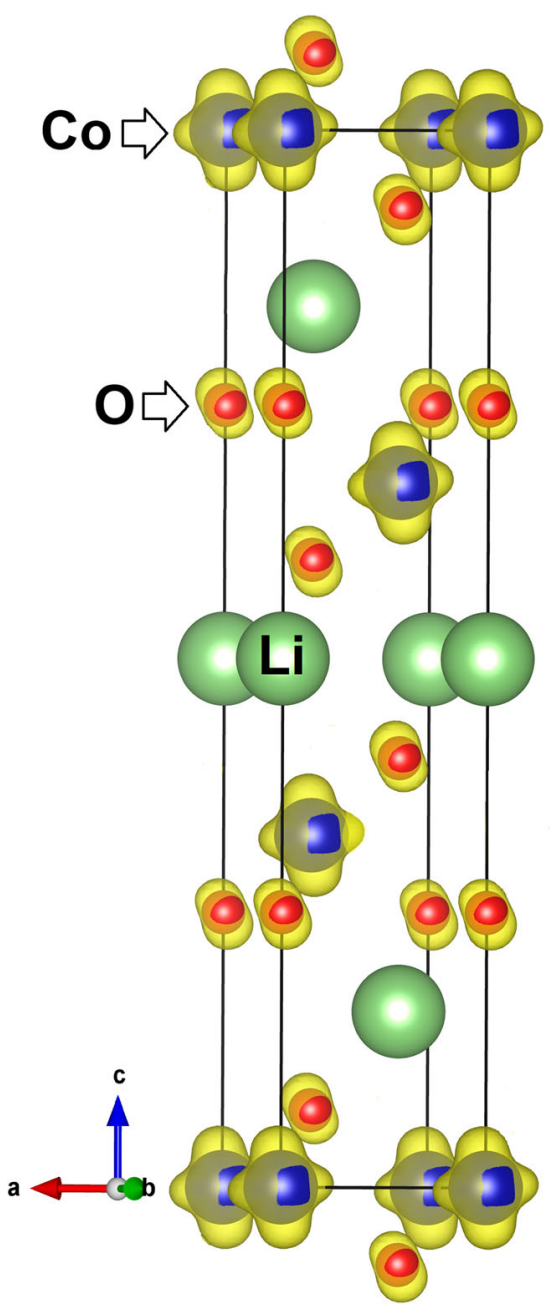

Fig. 2 Local softness 0.354 a.u. isosurface plot for $\mathrm{LiCoO}_{2}$. The $\mathrm{s}(\mathbf{r})$ isosurface is shown in yellow

system, before and after the structural relaxation. For all optimizations, the forces on the ions were below $0.01 \mathrm{eV} / \AA$ at each nucleus. All figures were done using VESTA [35].

Fig. 1 Local softness 0.354 a.u. isosurface plot for $\mathrm{LiFePO}_{4}$.

The $\mathrm{s}(\mathbf{r})$ isosurface is shown in yellow

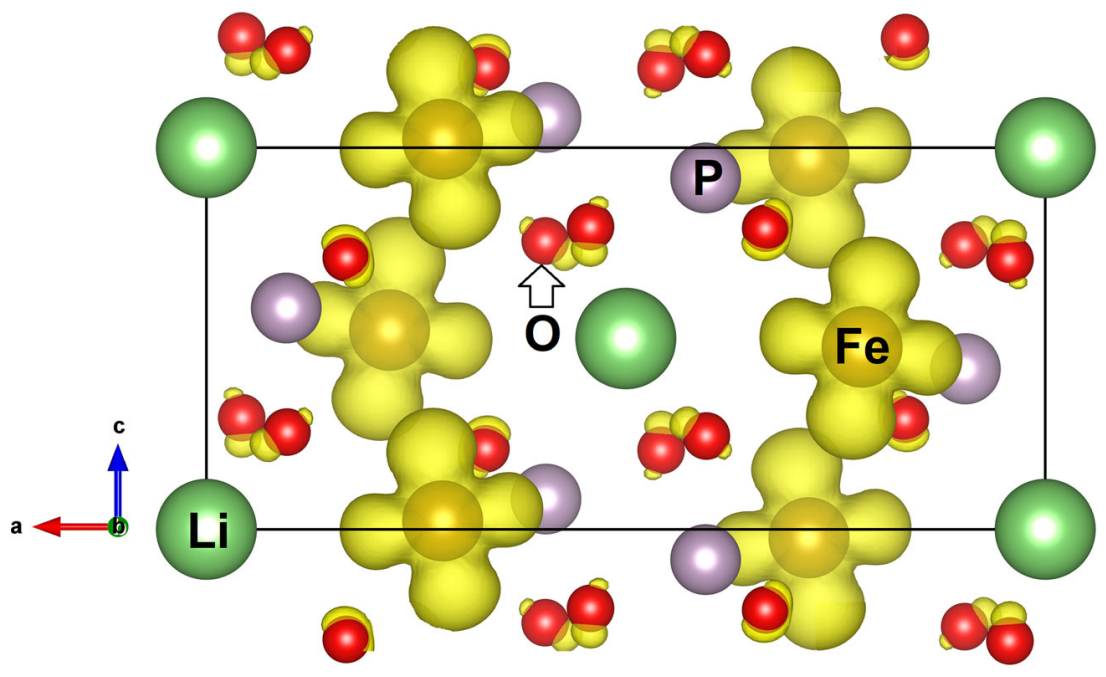




\section{Results}

\section{$\mathrm{LiFePO}_{4}$}

$\mathrm{LiFePO}_{4}$ is a cathode material now used in commercial Liion batteries [36, 37]. In this material, the electrochemical and stability properties can be explained considering that mainly the $\mathrm{Fe}$ atoms are involved in the charge-discharge process. As shown before [18], the projected-DOS of $\mathrm{Fe}$ atoms is the major contribution to the total DOS near the Fermi level. Figure 1 shows an isosurface of the local softness for $\mathrm{LiFePO}_{4}$. As can be seen, it is clear that the regions from where electrons are removed lie over the $\mathrm{Fe}$ atoms.

\section{$\mathrm{LiCoO}_{2}$}

Another commercial commonly used cathode material is $\mathrm{LiCoO}_{2}$. When analyzing the DOS near the Fermi level [18], it can be noticed that the states of the transition metal represent the major contribution to this region but the oxygen's states contribution increases compared to the one in $\mathrm{LiFePO}_{4}$. This observation is clearly established by the local softness when comparing Fig. 2 with Fig. 1. It has been observed that in this system the oxygen's states contribution increases with the degree of delithiation [18]. In practice, this limits the extraction of $\mathrm{Li}^{+}$to 0.5 ions per formula unit to avoid decomposition [38].

\section{$\mathrm{Li}_{2} \mathrm{Cu}_{0.5} \mathrm{M}_{0.5}, \mathrm{M}=\mathrm{Cu}, \mathrm{Ni}, \mathrm{Co}$}

$\mathrm{Li}_{2} \mathrm{CuO}_{2}$ is an interesting material with a high theoretical capacity as compared to other laminar transition metals oxides [39], from which in principle two lithium ions can be extracted. However, experimentally, it has been shown that this material has a high decrease of the capacity after the initial cycle and low stability [17]. This loss of capacity has been attributed to (1) structural transformations [40] and (2) side chemical reactions [17]. When analyzing the DOS of $\mathrm{Li}_{2} \mathrm{CuO}_{2}$ and his partially delithiated phase, $\mathrm{LiCuO}_{2}$, in both cases, the amount of oxygen electronic states near the Fermi level is bigger than those of copper; indeed, it is larger for $\mathrm{LiCuO}_{2}$ indicating that by continuing the delithiation process the release of $\mathrm{O}_{2}$ is highly probable giving rise to the appearance of other phases such as $\mathrm{CuO}$, inhibiting the reversibility and affecting the electrochemical performance [18]. Again, Fig. 3 shows that the local softness recovers the same information as the DOS analysis for these two compounds. One common way for a materials scientists to change the properties of known materials is to dope or form solid solutions from an existing one. In this context, on the experimental side, it has been determined that solid solutions of the type $\mathrm{Li}_{2} \mathrm{Cu}_{1-x} \mathrm{M}_{x} \mathrm{O}_{2} \quad(\mathrm{M}=\mathrm{Co}$, $\mathrm{Ni} ; \mathrm{x}=0.1-0.9)$, increase their capacity with respect to the pure lithium cuprate $\left(\mathrm{Li}_{2} \mathrm{CuO}_{2}\right)$ [41]. Effects of changing cationic environments around a particular redox pair in these materials have been remarked and it seems to be

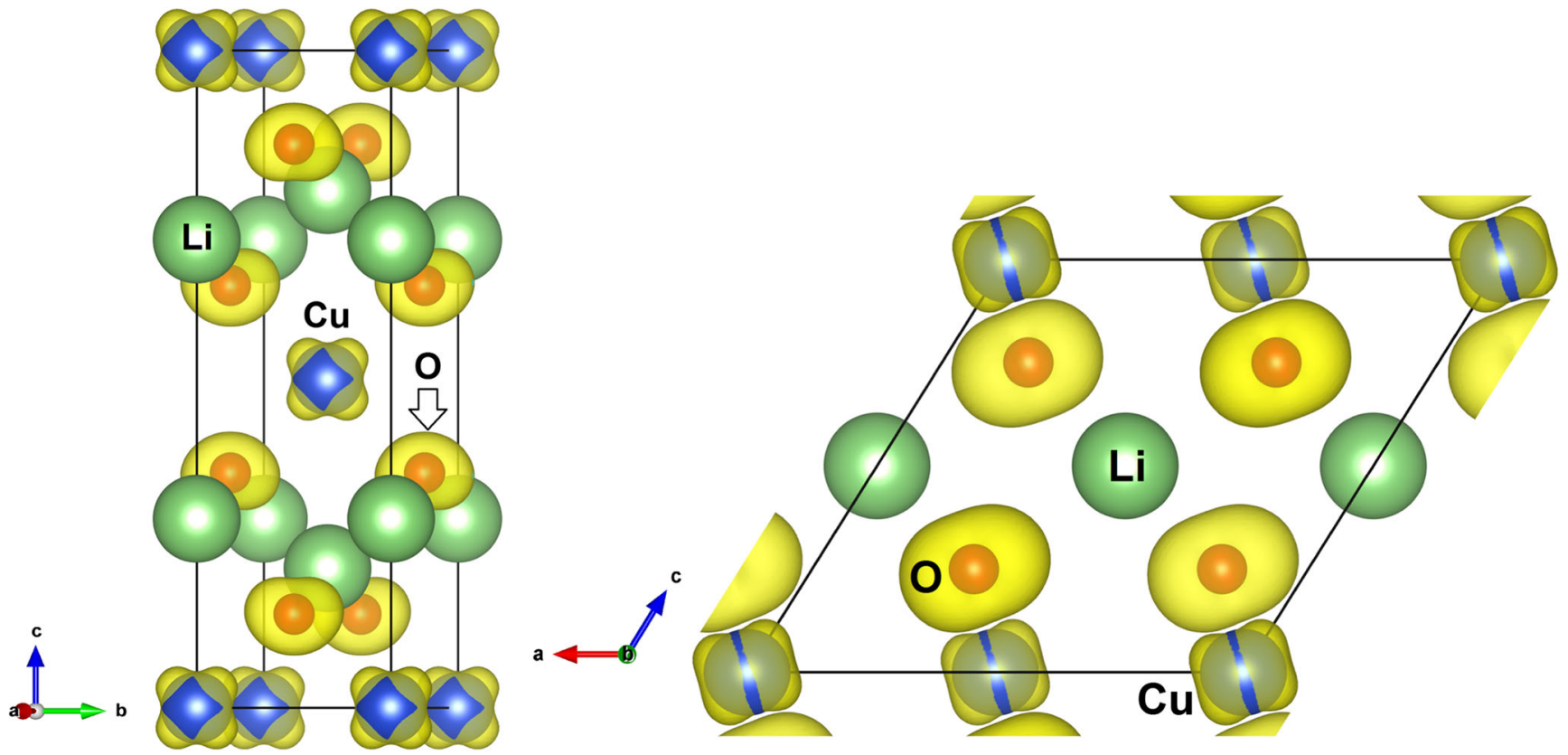

Fig. 3 Local softness 0.354 a.u. isosurface plots for $\mathrm{Li}_{2} \mathrm{CuO}_{2}($ left $)$ and $\mathrm{LiCuO}_{2}$ (right). The $\mathrm{s}(\mathbf{r})$ isosurfaces are shown in yellow 
Table 2 HSE06 total magnetization and Bader's atomic magnetic moment for each transition metal in $\mathrm{Li}_{2} \mathrm{CuO}_{2}, \mathrm{Li}_{2} \mathrm{Cu}_{0.5} \mathrm{Co}_{0.5} \mathrm{O}_{2}$ and $\mathrm{Li}_{2} \mathrm{Cu}_{0.5} \mathrm{Ni}_{0.5} \mathrm{O}_{2}$

\begin{tabular}{llll}
\hline & \multicolumn{2}{l}{ Magnetization } \\
\cline { 2 - 4 } System & $\mathrm{Cu}$ & $\mathrm{M}(\mathrm{M}=\mathrm{Co}$ or Ni) & Total \\
\hline $\mathrm{Li}_{2} \mathrm{CuO}_{2}$ & \pm 0.66 & & 0.00 \\
$\mathrm{Li}_{2} \mathrm{Cu}_{0.5} \mathrm{Co}_{0.5} \mathrm{O}_{2}$ & -0.66 & 2.70 & 2.00 \\
$\mathrm{Li}_{2} \mathrm{Cu}_{0.5} \mathrm{Ni}_{0.5} \mathrm{O}_{2}$ & -0.66 & 1.69 & 1.00 \\
\hline
\end{tabular}

a way of controlling potentials and the position of the oxygen band [42]. By evaluating the local softness, we can analyze the induced changes in the oxidation capabilities of oxygen sublattice in cuprates as several transition metal environments are changed near the cooper oxygen moiety. To perform this analysis, we proposed models of solid solutions for $\mathrm{Li}_{2} \mathrm{Cu}_{0.5} \mathrm{Co}_{0.5} \mathrm{O}_{2}$ and $\mathrm{Li}_{2} \mathrm{Cu}_{0.5} \mathrm{Ni}_{0.5} \mathrm{O}_{2}$. These models were built such that $50 \%$ of the $\mathrm{Cu}$ atoms were substituted by $\mathrm{Ni}$ or Co. As the objective is to test if the local softness is able to capture the electronic structure changes induced by the introduction of new chemical environments, the metal substitutions are performed in $\mathrm{Li}_{2} \mathrm{CuO}_{2}$ subject to the restriction of maintaining the crystal structure. Once each solid solution model reaches the minimum under restrictions, a full optimization of the structure, including cell parameters and atom positions, is done to test the stability of the structure. Table 2 shows the total magnetization and the atomic magnetic moment for the transition metal atoms in each compound obtained from a Bader population analysis [43, 44]. From these values, the following formal oxidation states can be derived: $\mathrm{Cu}^{2+}$ in $\mathrm{Li}_{2} \mathrm{CuO}_{2} ; \mathrm{Cu}^{2+}$ and $\mathrm{Co}^{2+}$ in $\mathrm{Li}_{2} \mathrm{Cu}_{0.5} \mathrm{Co}_{0.5} \mathrm{O}_{2}$ and $\mathrm{Cu}^{2+}$ and $\mathrm{Ni}^{2+}$ in $\mathrm{Li}_{2} \mathrm{Cu}_{0.5} \mathrm{Ni}_{0.5} \mathrm{O}_{2}$. A comparison of the lattice parameters and metal-oxygen bond distances is displayed in Tables 3 and 4 . The changes induced by ion substitution in the cell parameter follow the ionic

Table 3 Computed structural parameters of $\mathrm{Li}_{2} \mathrm{CuO}_{2}, \mathrm{Li}_{2} \mathrm{Cu}_{0.5} \mathrm{Co}_{0.5}$ $\mathrm{O}_{2}$ and $\mathrm{Li}_{2} \mathrm{Cu}_{0.5} \mathrm{Ni}_{0.5} \mathrm{O}_{2}$

\begin{tabular}{lcccc}
\hline System & $\mathrm{a}(\AA)$ & $\mathrm{b}(\AA)$ & $\mathrm{c}(\AA)$ & Volume $\left(\AA^{3}\right)$ \\
\hline $\mathrm{Li}_{2} \mathrm{CuO}_{2}$ & 3.701 & 2.875 & 9.396 & 99.977 \\
& $(3.660)^{\mathrm{a}}$ & $(2.861)^{\mathrm{a}}$ & $(9.389)^{\mathrm{a}}$ & 98.315 \\
& $(3.661)^{\mathrm{b}}$ & $(2.866)^{\mathrm{b}}$ & $(9.397)^{\mathrm{b}}$ & 98.597 \\
$\mathrm{Li}_{2} \mathrm{Cu}_{0.5} \mathrm{Co}_{0.5} \mathrm{O}_{2}$ & 3.694 & 2.906 & 9.490 & 101.873 \\
$\mathrm{Li}_{2} \mathrm{Cu}_{0.5} \mathrm{Ni}_{0.5} \mathrm{O}_{2}$ & 3.696 & 2.890 & 9.394 & 100.341 \\
& $(3.692)^{\mathrm{a}}$ & $(2.827)^{\mathrm{a}}$ & $(9.238)^{\mathrm{a}}$ & 96.420
\end{tabular}

Experimental parameters when available are also shown in parenthesis ${ }^{a}$ Experimental Parameters Ref. [45]

${ }^{\mathrm{b}}$ Experimental Parameters Ref. [50]
Table 4 Predicted $\mathrm{M}-\mathrm{O}$ bond distances $(\mathrm{M}=\mathrm{Cu}$, Co or $\mathrm{Ni})$ for $\mathrm{Li}_{2} \mathrm{CuO}_{2}, \mathrm{Li}_{2} \mathrm{Cu}_{0.5} \mathrm{Co}_{0.5} \mathrm{O}_{2}$ and $\mathrm{Li}_{2} \mathrm{Cu}_{0.5} \mathrm{Ni}_{0.5} \mathrm{O}_{2}$

\begin{tabular}{lcll} 
Distance d, $(\AA)$ & $\mathrm{Li}_{2} \mathrm{CuO}_{2}$ & $\mathrm{Li}_{2} \mathrm{Cu}_{0.5} \mathrm{Co}_{0.5} \mathrm{O}_{2}$ & $\mathrm{Li}_{2} \mathrm{Cu}_{0.5} \mathrm{Ni}_{0.5} \mathrm{O}_{2}$ \\
\hline $\mathrm{M}-\mathrm{O}$ & 1.97 & 2.00 & 1.97 \\
& $(1.96)^{\mathrm{a}}$ & & $(1.92)^{\mathrm{b}}$ \\
$\mathrm{Cu}-\mathrm{O}$ & 1.97 & 1.97 & 1.97 \\
& $(1.96)^{\mathrm{a}}$ & & $(1.92)^{\mathrm{b}}$ \\
\hline
\end{tabular}

Distances are taken from the metal atom to the nearest oxygen atom. Experimental information when available is also shown in parenthesis

${ }^{a}$ Experimental Parameters Ref. [50]

${ }^{\mathrm{b}}$ Experimental Parameters Ref. [45]

radii behavior, $\mathrm{Ni}^{2+}<\mathrm{Cu}^{2+}<\mathrm{Co}^{2+}$. To analyze the changes induced in the electronic structure of lithium cuprate, when a nearby chemical environment is changed by substitution with other transition metal maintaining the crystal structure, a comparison of the projected-DOS for $\mathrm{Li}_{2} \mathrm{CuO}_{2}, \mathrm{Li}_{2} \mathrm{Cu}_{0.5} \mathrm{Co}_{0.5} \mathrm{O}_{2}$ and $\mathrm{Li}_{2} \mathrm{Cu}_{0.5} \mathrm{Ni}_{0.5} \mathrm{O}_{2}$ is displayed in Fig. 4. In Fig. 4a, the well-known feature [18] of the overlap between the copper and oxygen related occupied states close to the Fermi energy is evident. This characteristic is linked to oxygen evolution upon oxidation of the material. When half of the copper atoms are substituted by cobalt atoms in the structure, the cobaltassociated states occupy the place of those of copper and are those states the predominant ones among the occupied states close to the Fermi energy (see Fig. 4b); it is important to notice that there is also a significant oxygen states contribution in that region of the projected-DOS. As one may see in Fig. 4c, when the substitution is by the nickel atom, the projected-DOS has a similar behavior to the one of the pure cuprate: oxygen states prevail among the occupied states close to the Fermi level; the nickel sates are present too in that region but the differences are not as strong as for the case of cobalt. In this case, the copper states are pushed towards lower energies. Figure 5 shows an isosurface of the local softness for $\mathrm{Li}_{2} \mathrm{Cu}_{0.5} \mathrm{Co}_{0.5} \mathrm{O}_{2}$ and $\mathrm{Li}_{2} \mathrm{Cu}_{0.5} \mathrm{Ni}_{0.5} \mathrm{O}_{2}$. It is clear from these plots that the same conclusions can be drawn regarding the predominant states near the Fermi level. Also, the local softness suggests that in these solid solutions, the voltage should be dominated by the incorporated transition metal atom in agreement with the experimental results at least for $\mathrm{Li}_{2} \mathrm{Cu}_{0.5} \mathrm{Ni}_{0.5} \mathrm{O}_{2}$ [45].

As we already mentioned, the information provided by the analysis of the projected-DOS and the local softness is equivalent. However, it should be highlighted that the analysis of local softness does not necessarily require a projection procedure by region or atom because the isosurface plots clearly distinguish the regional charge transfer capabilities for different systems with a quantitative character. 
Fig. 4 Projected-DOS for oxygen and all transition metal atoms in $\mathbf{a ~} \mathrm{Li}_{2} \mathrm{CuO}_{2}$, b $\mathrm{Li}_{2} \mathrm{Cu}_{0.5} \mathrm{Co}_{0.5} \mathrm{O}_{2}$ and $\mathbf{c}$ $\mathrm{Li}_{2} \mathrm{Cu}_{0.5} \mathrm{Ni}_{0.5} \mathrm{O}_{2}$. The dashed line indicates the Fermi Level in each system. The Fermi level and the violet dashed line enclose the energy range where the total density of states integrates to 1 electron. All projected DOS were obtained from a calculation performed using the HSE06 functional

Fig. 5 Local softness 0.354 a.u. isosurface plots for $\mathrm{Li}_{2} \mathrm{Cu}_{0.5} \mathrm{Co}_{0.5} \mathrm{O}_{2}$ (left) and $\mathrm{Li}_{2} \mathrm{Cu}_{0.5} \mathrm{Ni}_{0.5} \mathrm{O}_{2}$ (right). The $\mathrm{s}(\mathbf{r})$ isosurfaces are shown in yellow
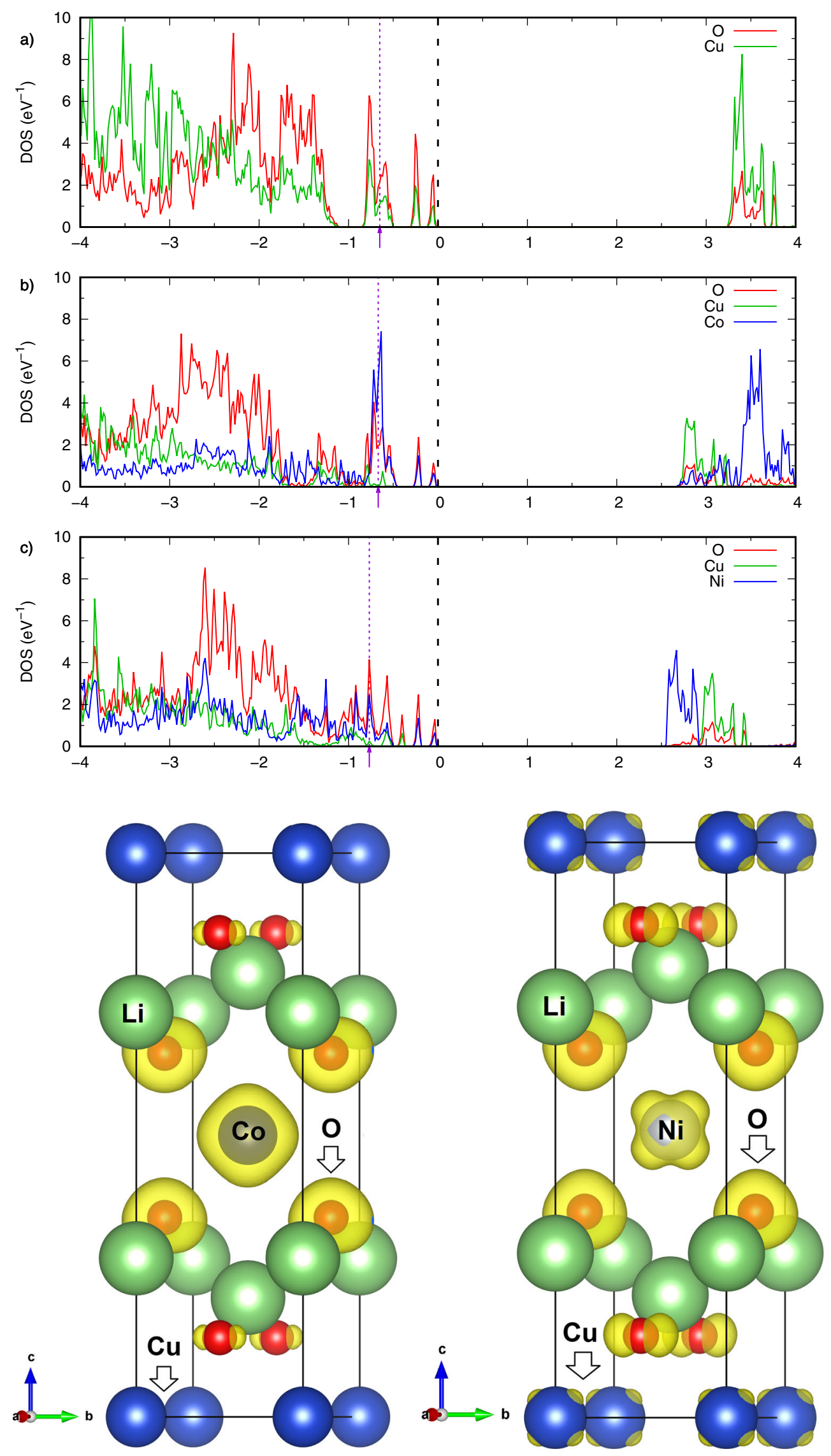


\section{Conclusions}

We have shown that the local softness is an alternative property to look at for studying the electronic states near the Fermi level in cathode materials. Without the need of any projection, the local softness provides in a more direct way the same information that can be obtained by analyzing the DOS.

Acknowledgements It is a pleasure to dedicate this work to celebrate the 60th birthday of Professor Pratim Kumar Chattaraj; the scientific contributions of Prof. Chattaraj in the field of chemical reactivity within the DFT framework have been seminal and inspiring. We thank Issis Romero, Jorge Vázquez, Guadalupe Ramos and Ignacio González for many fruitful discussions. The authors gratefully acknowledge the computing time granted by LANCAD and CONACYT on the supercomputer Yoltla at LSVP UAM-Iztapalapa. We also thank CONACYT for financial support through Projects 237343 and 286013 and for LIPR's PhD scholarship (No. 398738).

\section{References}

1. Aydinol MK, Kohan AF, Ceder G, Cho K, Joannopoulos J (1997) $\mathrm{Ab}$ initio study of lithium intercalation in metal oxides and metal dichalcogenides. Phys Rev B 56:1354-1365

2. Seo DH, Lee J, Urban A, Malik R, Kang S, Ceder G (2016) The structural and chemical origin of the oxygen redox activity in layered and cation-disordered Li-excess cathode materials. Nat Chem 8:692-7

3. McCalla E, Abakumov AM, Saubanère M, Foix D, Berg EJ, Rousse G, Doublet M-L, Gonbeau D, Novák P, Van Tendeloo G (2015) Visualization of O-O peroxo-like dimers in high-capacity layered oxides for $\mathrm{Li}$-ion batteries. Science 350:1516-1521

4. Sathiya M, Rousse G, Ramesha K, Laisa CP, Vezin H, Sougrati MT, Doublet ML, Foix D, Gonbeau D, Walker W, Prakash AS, Ben Hassine M, Dupont L, Tarascon JM (2013) Reversible anionic redox chemistry in high-capacity layered-oxide electrodes. Nat Mater 12:827-35

5. Koga H, Croguennec L, Ménétrier M, Douhil K, Belin S, Bourgeois L, Suard E, Weill F, Delmas C (2013) Reversible oxygen participation to the redox processes revealed for $\mathrm{Li}_{1.20} \mathrm{Mn}_{0.54} \mathrm{Co}_{0.13} \mathrm{Ni}_{0.13} \mathrm{O}_{2}$. J Electrochem Soc 160:A786-A792

6. Yabuuchi N, Takeuchi M, Nakayama M, Shiiba H, Ogawa M, Nakayama K, Ohta T, Endo D, Ozaki T, Inamasu T, Sato K, Komaba S (2015) High-capacity electrode materials for rechargeable lithium batteries: $\mathrm{Li}_{3} \mathrm{NbO}_{4}$-based system with cation-disordered rocksalt structure. Proc Natl Acad Sci USA 112:7650-7655

7. McCalla E, Sougrati MT, Rousse G, Berg EJ, Abakumov A, Recham N, Ramesha K, Sathiya M, Dominko R, Van Tendeloo G, Novák P, Tarascon J-M (2015) Understanding the roles of anionic redox and oxygen release during electrochemical cycling of lithium-rich layered $\mathrm{Li}_{4} \mathrm{FeSbO}_{6}$. J Am Chem Soc 137:48044814

8. Sathiya M, Ramesha K, Rousse G, Foix D, Gonbeau D, Prakash AS, Doublet ML, Hemalatha K, Tarascon JM (2013) Highperformance $\mathrm{Li}_{2} \mathrm{Ru}_{1-y} \mathrm{Mn}_{y} \mathrm{O}_{3}(0.2 \leq \mathrm{y} \leq 0.8)$ cathode materials for rechargeable lithium-ion batteries: their understanding. Chem Mater 25:1121-1131

9. Koga H, Croguennec L, Ménétrier M, Mannessiez P, Weill F, Delmas C (2013) Different oxygen redox participation for bulk and surface: a possible global explanation for the cycling mechanism of $\mathrm{Li}_{1.20} \mathrm{Mn}_{0.54} \mathrm{Co}_{0.13} \mathrm{Ni}_{0.13} \mathrm{O}_{2}$. J Power Sources 236:250-258

10. Armstrong AR, Holzapfel M, Novák P, Johnson CS, Kang S-H, Thackeray MM, Bruce PG (2006) Demonstrating oxygen loss and associated structural reorganization in the lithium battery cathode $\mathrm{Li}\left[\mathrm{Ni}_{0.2} \mathrm{Li}_{0.2} \mathrm{Mn}_{0.6}\right] \mathrm{O}_{2}$. J Am Chem Soc 128:8694-8698

11. Marusczyk A, Albina J-M, Hammerschmidt T, Drautz R, Eckl T, Henkelman G (2017) Oxygen activity and peroxide formation as charge compensation mechanisms in $\mathrm{Li}_{2} \mathrm{MnO}_{3}$. J Mater Chem A 5:15183-15190

12. Xu B, Fell CR, Chi M, Meng YS (2011) Identifying surface structural changes in layered Li-excess nickel manganese oxides in high-voltage lithium ion batteries: a joint experimental and theoretical study. Energy Environ Sci 4:2223-2233

13. Fell CR, Qian D, Carroll KJ, Chi M, Jones JL, Meng YS (2013) Correlation between oxygen vacancy, microstrain, and cation distribution in lithium-excess layered oxides during the first electrochemical cycle. Chem Mater 25:1621-1629

14. Gent WE, Lim K, Liang Y, Li Q, Barnes T, Ahn S-J, Stone $\mathrm{KH}$, McIntire M, Hong J, Song JH, Li Y, Mehta A, Ermon S, Tyliszczak T, Kilcoyne D, Vine D, Park J-H, Doo S-K, Toney MF, Yang W, Prendergast D, Chueh WC (2017) Coupling between oxygen redox and cation migration explains unusual electrochemistry in lithium-rich layered oxides. Nat Commun 8:2091

15. Qian D, Xu B, Chi M, Meng YS (2014) Uncovering the roles of oxygen vacancies in cation migration in lithium excess layered oxides. Phys Chem Chem Phys 16:14665-14668

16. Wang Q, Ping P, Zhao X, Chu G, Sun J, Chen C (2012) Thermal runaway caused fire and explosion of lithium ion battery. J Power Sources 208:210-224

17. Love CT, Dmowski W, Johannes MD, Swider-Lyons KE (2011) Structural originations of irreversible capacity loss from highly lithiated copper oxides. J Solid State Chem 184:2412-2419

18. Johannes M, Swider-Lyons K, Love CT (2016) Oxygen character in the density of states as an indicator of the stability of Li-ion battery cathode materials. Solid State Ion 286:83-89

19. Yang W, Parr RG (1985) Hardness, softness, and the Fukui function in the electronic theory of metals and catalysis. Proc Natl Acad Sci USA 82:6723-6726

20. Cohen MH, Ganduglia-Pirovano MV, Kudrnovský J (1994) Electronic and nuclear chemical reactivity. J Chem Phys 101:8988-8997

21. Geerlings P, De Proft F, Langenaeker W (2003) Conceptual density functional theory. Chem Rev 103:1793-1874

22. Cohen MH, Ganduglia-Pirovano MV, Kudrnovský J (1995) Reactivity kernels, the normal modes of chemical reactivity, and the hardness and softness spectra. J Chem Phys 103:3543-3551

23. Cohen MH, Ganduglia-Pirovano MV, Kudrnovský J (1994) Orbital symmetry, reactivity, and transition metal surface chemistry. Phys Rev Lett 72:3222-3225

24. Wilke S, Cohen MH, Scheffler M (1996) Local isoelectronic reactivity of solid surfaces. Phys Rev Lett 77:1560-1563

25. Brommer KD, Galván M, Dal Pino A, Joannopoulos JD (1994) Theory of adsorption of atoms and molecules on $\mathrm{Si}(111)-(7 \times 7)$. Surf Sci 314:57-70

26. Cárdenas C, De Proft F, Chamorro E, Fuentealba P, Geerlings P (2008) Theoretical study of the surface reactivity of alkaline earth oxides: local density of states evaluation of the local softness. J Chem Phys 128:034708

27. Pino-Rios R, Yañez O, Inostroza D, Ruiz L, Cardenas C, Fuentealba P, Tiznado W (2017) Proposal of a simple and effective local reactivity descriptor through a topological analysis of an orbital-weighted Fukui function. J Comput Chem 38:481-488 
28. Cárdenas C, Muñoz M, Contreras J, Ayers PW, Gómez T, Fuentealba P (2018) Understanding chemical reactivity in extended systems: exploring models of chemical softness in carbon nanotubes. Acta Phys -Chim Sin 34:631-638

29. Kresse G, Furthmüller J (1996) Efficient iterative schemes for ab initio total-energy calculations using a plane-wave basis set. Phys Rev B 54:11169-11186

30. Kresse G, Joubert D (1999) From ultrasoft pseudopotentials to the projector augmented-wave method. Phys Rev B 59:1758-1775

31. Blöchl PE (1994) Projector augmented-wave method. Phys Rev B 50:17953-17979

32. Dudarev SL, Botton GA, Savrasov SY, Humphreys CJ, Sutton AP (1998) Electron-energy-loss spectra and the structural stability of nickel oxide: an LSDA+U study. Phys Rev B 57:15051509

33. Heyd J, Scuseria GE, Ernzerhof M (2003) Hybrid functionals based on a screened Coulomb potential. J Chem Phys 118:82078215

34. Johannes MD, Hoang K, Allen JL, Gaskell K (2012) Hole polaron formation and migration in olivine phosphate materials. Phys Rev B 85:115106

35. Momma K, Izumi F (2011) VESTA 3 for three-dimensional visualization of crystal, volumetric and morphology data. J Appl Crystallogr 44:1272-1276

36. Padhi AK, Nanjundaswamy KS, Goodenough JB (1997) Phosphoolivines as positive-electrode materials for rechargeable lithium batteries. J Electrochem Soc 144:1188-1194

37. Eftekhari A (2017) LiFePO4/C nanocomposites for lithium-ion batteries. J Power Sources 343:395-411

38. Gupta R, Manthiram A (1996) Chemical extraction of lithium from layered $\mathrm{LiCoO}_{2}$. J Solid State Chem 121:483-491

39. Ramos-Sanchez G, Romero-Ibarra IC, Vazquez-Arenas J, Tapia C, Aguilar-Eseiza N, Gonzalez I (2017) Controlling $\mathrm{Li}_{2} \mathrm{CuO}_{2}$ single phase transition to preserve cathode capacity and cyclability in Li-ion batteries. Solid State Ion 303:89-96
40. Imanishi N, Shizuka K, Ikenishi T, Matsumura T, Hirano A, Takeda Y (2006) Preparation and electrochemical properties of a $\mathrm{Li}_{2} \mathrm{CuO}_{2}-\mathrm{Li}_{2} \mathrm{NiO}_{2}$ solid solution as a lithium-intercalation electrode. Solid State Ion 177:1341-1346

41. Setiawati E, Hayashi M, Tsuda M, Hayashi K, Kobayashi R (2014) Structural changes and electrochemical properties of $\mathrm{Li}_{2} \mathrm{Cu}_{1-x} \mathrm{M}_{x} \mathrm{O}_{2}$ for lithium secondary batteries. Solid State Ion 262:115-119

42. Goodenough JB, Kim Y (2010) Challenges for rechargeable Li batteries. Chem Mater 22:587-603

43. Bader R (1994) Atoms in molecules: a quantum theory. Clarendon Press, Oxford

44. Tang W, Sanville E, Henkelman G (2009) A grid-based Bader analysis algorithm without lattice bias. J Phys Condens Matter 21:084204

45. Ruther RE, Zhou H, Dhital C, Saravanan K, Kercher AK, Chen G, Huq A, Delnick FM, Nanda J (2015) Synthesis, structure, and electrochemical performance of high capacity $\mathrm{Li}_{2} \mathrm{Cu}_{0.5} \mathrm{Ni}_{0.5} \mathrm{O}_{2}$ cathodes. Chem Mater 27:6746-6754

46. Zhou F, Cococcioni M, Marianetti CA, Morgan D, Ceder G (2004) First-principles prediction of redox potentials in transition-metal compounds with LDA $+U$. Phys Rev B 70:235121

47. Xiang HJ, Lee C, Whangbo MH (2007) Absence of a spiral magnetic order in $\mathrm{Li}_{2} \mathrm{CuO}_{2}$ containing one-dimensional $\mathrm{CuO}_{2}$ ribbon chains. Phys Rev B 76:220411

48. Chevrier VL, Ong SP, Armiento R, Chan MKY, Ceder G (2010) Hybrid density functional calculations of redox potentials and formation energies of transition metal compounds. Phys Rev B 82:075122

49. Hinuma Y, Meng YS, Kang K, Ceder G (2007) Phase transitions in the $\mathrm{LiNi}_{0.5} \mathrm{Mn}_{0.5} \mathrm{O}_{2}$ system with temperature. Chem Mater 19:1790-1800

50. Chung EML, McIntyre GJ, Paul DM, Balakrishnan G, Lees MR (2003) Oxygen moment formation and canting in $\mathrm{Li}_{2} \mathrm{CuO}_{2}$. Phys Rev B 68:144410 


\section{REFERENCIAS}

[1] Liu, C.; Neale, Z. G.; Cao, G. Understanding electrochemical potentials of cathode materials in rechargeable batteries. Mater. Today, 19:109-123, 2016.

[2] Meng, Y. S.; Arroyo-de Dompablo, M. E. Recent advances in first principles computational research of cathode materials for lithium-ion batteries. Acc. Chem. Res., 46:1171-1180, 2013.

[3] Deng, D. Li-ion batteries: basics, progress, and challenges. Energy Sci. Eng., 3:385418, 2015.

[4] Winter, M.; Brodd, R. J. What are batteries, fuel cells, and supercapacitors? Chem. Rev., 104:4245-4270, 2004.

[5] Aydinol, M. K.; Kohan, A. F.; Ceder, G.; Cho, K.; Joannopoulos, J. Ab initio study of lithium intercalation in metal oxides and metal dichalcogenides. Phys. Rev. B, 56:1354-1365, 1997.

[6] Ceder, G.; Aydinol, M. K.; Kohan, A. F. Application of first-principles calculations to the design of rechargeable Li-batteries. Comput. Mater. Sci., 8:161-169, 1997.

[7] Whittingham, M. S. Lithium batteries and cathode materials. Chem. Rev., 104:42714302, 2004.

[8] Love, C. T.; Dmowski, W.; Johannes, M. D.; Swider-Lyons, K. E. Structural originations of irreversible capacity loss from highly lithiated copper oxides. J. Solid State Chem., 184:2412-2419, 2011.

[9] Raekelboom, E. A.; Hector, A. L.; Weller, M. T.; Owen, J. R. Electrochemical properties and structures of the mixed-valence lithium cuprates $\mathrm{Li}_{3} \mathrm{Cu}_{2} \mathrm{O}_{4}$ and $\mathrm{Li}_{2} \mathrm{NaCu}_{2} \mathrm{O}_{4}$. J. Power Sources, 97-98:465-468, 2001.

[10] Gupta, R.; Manthiram, A. Chemical extraction of lithium from layered $\mathrm{LiCoO}_{2} . J$. Solid State Chem., 121:483-491, 1996. 
[11] Jang, D. H.; Shin, Y. J.; Oh, S. M. Dissolution of spinel oxides and capacity losses in $4 \mathrm{~V} \mathrm{Li} / \mathrm{Li}_{x} \mathrm{Mn}_{2} \mathrm{O}_{4}$ cells. J. Electrochem. Soc., 143:2204-2211, 1996.

[12] Padhi, A. K.; Nanjundaswamy, K. S.; Goodenough, J. B. Phospho-olivines as positive-electrode materials for rechargeable lithium batteries. J. Electrochem. Soc., 144:1188-1194, 1997.

[13] Takahashi, Y.; Tode, S.; Kinoshita, A.; Fujimoto, H.; Nakane, I.; Fujitani, S. Development of Lithium-Ion batteries with a $\mathrm{LiCoO}_{2}$ cathode toward high capacity by elevating charging potential. J. Electrochem. Soc., 155:A537-A541, 2008.

[14] Kim, M.; Jung, Y.; Kang, B. High electrochemical performance of $3.9 \mathrm{~V} \mathrm{LiFeSO}_{4} \mathrm{~F}$ directly synthesized by a scalable solid-state reaction within $1 \mathrm{~h}$. J. Mater. Chem. A, 3:7583-7590, 2015.

[15] Hautier, G.; Jain, A.; Ong, S. P.; Kang, B.; Moore, C.; Doe, R.; Ceder, G. Phosphates as lithium-ion battery cathodes: An evaluation based on high-throughput ab initio calculations. Chem. Mater., 23:3495-3508, 2011.

[16] Ruther, R. E.; Zhou, H.; Dhital, C.; Saravanan, K.; Kercher, A. K.; Chen, G.; Huq, A.; Delnick, F. M.; Nanda, J. Synthesis, structure, and electrochemical performance of high capacity $\mathrm{Li}_{2} \mathrm{Cu}_{0.5} \mathrm{Ni}_{0.5} \mathrm{O}_{2}$ cathodes. Chem. Mater., 27:6746-6754, 2015.

[17] Giri, S.; Chudo, H.; Nakamura, H.; Shiga, M. Interchain interactions of $\mathrm{Li}_{2} \mathrm{CuO}_{2}$ probed by ${ }^{7}$ Li NMR. J. Alloys Compd., 326:61-64, 2001.

[18] Ramos-Sanchez, G.; Romero-Ibarra, I. C.; Vazquez-Arenas, J.; Tapia, C.; AguilarEseiza, N.; Gonzalez, I. Controlling $\mathrm{Li}_{2} \mathrm{CuO}_{2}$ single phase transition to preserve cathode capacity and cyclability in Li-ion batteries. Solid State Ionics, 303:89-96, 2017.

[19] Arai, H.; Okada, S.; Sakurai, Y.; Yamaki, J.-I. Electrochemical and structural study of $\mathrm{Li}_{2} \mathrm{CuO}_{2}, \mathrm{LiCuO}_{2}$ and $\mathrm{NaCuO}_{2}$. Solid State Ionics, 106:45-53, 1998.

[20] Setiawati, E.; Hayashi, M.; Tsuda, M.; Hayashi, K.; Kobayashi, R. Structural changes and electrochemical properties of $\mathrm{Li}_{2} \mathrm{Cu}_{1-x} \mathrm{M}_{x} \mathrm{O}_{2}$ for lithium secondary batteries. Solid State Ionics, 262:115-119, 2014.

[21] Ruther, R. E.; Samuthira Pandian, A.; Yan, P.; Weker, J. N.; Wang, C.; Nanda, J. Structural transformations in high-capacity $\mathrm{Li}_{2} \mathrm{Cu}_{0.5} \mathrm{Ni}_{0.5} \mathrm{O}_{2}$ cathodes. Chem. Mater., 29:2997-3005, 2017.

[22] Love, C. T.; Johannes, M. D.; Stux, A. M.; Swider-Lyons, K. Characterization and electrochemical properties of $\mathrm{Li}_{2} \mathrm{Cu}_{0.5} \mathrm{Ni}_{0.4} \mathrm{M}_{0.1} \mathrm{O}_{2}$ Lithium-ion battery cathodes. ECS Trans., 16:27-35, 2009. 
[23] Seo, D. H.; Lee, J.; Urban, A.; Malik, R.; Kang, S.; Ceder, G. The structural and chemical origin of the oxygen redox activity in layered and cation-disordered $\mathrm{Li}$ excess cathode materials. Nat. Chem., 8:692-697, 2016.

[24] Johannes, M. D.; Swider-Lyons, K.; Love, C. T. Oxygen character in the density of states as an indicator of the stability of Li-ion battery cathode materials. Solid State Ionics, 286:83-89, 2016.

[25] Sathiya, M.; Rousse, G.; Ramesha, K.; Laisa, C. P.; Vezin, H.; Sougrati, M. T.; Doublet, M-L.; Foix, D.; Gonbeau, D.; Walker, W.; Prakash, A. S.; Ben Hassine, M.; Dupont, L.; Tarascon, J-M. Reversible anionic redox chemistry in high-capacity layered-oxide electrodes. Nat. Mater., 12:827-835, 2013.

[26] Koga, H.; Croguennec, L.; Ménétrier, M.; Douhil, K.; Belin, S.; Bourgeois, L.; Suard, E.; Weill, F.; Delmas, C. Reversible oxygen participation to the redox processes revealed for $\mathrm{Li}_{1.20} \mathrm{Mn}_{0.54} \mathrm{Co}_{0.13} \mathrm{Ni}_{0.13} \mathrm{O}_{2}$. J. Electrochem. Soc., 160:A786-A792, 2013.

[27] Yabuuchi, N.; Takeuchi, M.; Nakayama, M.; Shiiba, H.; Ogawa, M.; Nakayama, K.; Ohta, T.; Endo, D.; Ozaki, T.; Inamasu, T.; Sato, K.; Komaba, S. High-capacity electrode materials for rechargeable lithium batteries: $\mathrm{Li}_{3} \mathrm{NbO}_{4}$-based system with cation-disordered rocksalt structure. Proc. Natl. Acad. Sci. U.S.A., 112:7650-7655, 2015.

[28] McCalla, E.; Sougrati, M. T.; Rousse, G.; Berg, E. J.; Abakumov, A.; Recham, N.; Ramesha, K.; Sathiya, M.; Dominko, R.; Van Tendeloo, G.; Novák, P.; Tarascon, J.-M. Understanding the roles of anionic redox and oxygen release during electrochemical cycling of lithium-rich layered $\mathrm{Li}_{4} \mathrm{FeSbO}_{6}$. J. Am. Chem. Soc., 137:4804-4814, 2015.

[29] Sathiya, M.; Ramesha, K.; Rousse, G.; Foix, D.; Gonbeau, D.; Prakash, A. S.; Doublet,

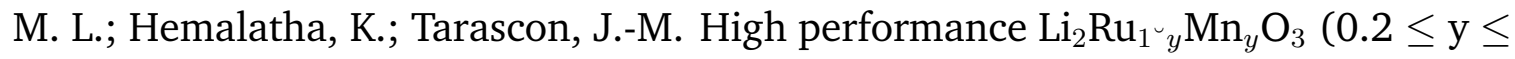
0.8 ) cathode materials for rechargeable lithium-ion batteries: Their understanding. Chem. Mater., 25:1121-1131, 2013.

[30] Wang, Q.; Ping, P.; Zhao, X.; Chu, G.; Sun, J.; Chen, C. Thermal runaway caused fire and explosion of lithium ion battery. J. Power Sources, 208:210-224, 2012.

[31] Schrödinger, E. Quantisierung als eigenwertproblem. Ann. Phys., 384:361-376, 1926.

[32] Dronskowski, R. Computational Chemistry of Solid State Materials. WILEY-VCH Verlag GmbH \& Co. KGaA, 2005.

[33] Parr, R. G.; Yang, W. Density-functional theory of atoms and molecules. Oxford University Press, Inc., 1989. 
[34] Koch, W.; Holthausen, M. C. A Chemist's Guide to Density Functional Theory. WileyVCH Verlag GmbH, 2001.

[35] Martin, R. M. Electronic Structure: Basic Theory and Practical Methods. Cambridge University Press, 2004.

[36] Perdew, J. P.; Schmidt, K. Jacob's ladder of density functional approximations for the exchange-correlation energy. AIP Conf. Proc., 577:1-20, 2001.

[37] Payne, M. C.; Teter, M. P.; Allan, D. C.; Arias, T. A.; Joannopoulos, J. D. Iterative minimization techniques for ab initio total-energy calculations: molecular dynamics and conjugate gradients. Rev. Mod. Phys., 64:1045-1097, 1992.

[38] Kaxiras, E. Atomic and Electronic Structure of Solids. Cambridge University Press, 2003.

[39] Szabo, A.; Ostlund, N. S. Modern Quantum Chemistry: Introduction to Advanced Electronic Structure Theory. Dover Publications, Inc., 1996.

[40] Hohenberg, P.; Kohn, W. Inhomogeneous electron gas. Phys. Rev., 136:B864-B871, 1964.

[41] Kohn, W.; Sham, L. J. Self-consistent equations including exchange and correlation effects. Phys. Rev., 140:A1133-A1138, 1965.

[42] Jensen, F. Introduction to Computational Chemistry, Second Edition. John Wiley \& Sons Ltd, 2007.

[43] Thijssen, J. M. Computational Physics, Second Edition. Cambridge University Press, 2007.

[44] Zhang, I. Y.; Xu, X. A New-Generation Density Functional: Towards Chemical Accuracy for Chemistry of Main Group Elements. Springer-Verlag Berlin Heidelberg, 2014.

[45] Perdew, J. P.; Burke, K.; Ernzerhof, M. Generalized gradient approximation made simple. Phys. Rev. Lett., 77:3865-3868, 1996.

[46] Perdew, J. P.; Burke, K.; Ernzerhof, M. Generalized gradient approximation made simple [Phys. Rev. Lett. 77, 3865 (1996)]. Phys. Rev. Lett., 78:1396-1396, 1997.

[47] Himmetoglu, B.; Floris, A.; de Gironcoli, S.; Cococcini, M. Hubbard-Corrected DFT energy Functionals: The LDA+U description of correlated systems. Int. J. Quantum Chem., 114:14-49, 2014.

[48] Hüfner, S. Electronic structure of $\mathrm{NiO}$ and related 3d-transition-metal compounds. Adv. Phys., 43:183-356, 1994. 
[49] Hubbard, J. Electron correlations in narrow energy bands. Proc. R. Soc. London A, 276:238-257, 1963.

[50] Hubbard, J. Electron correlations in narrow energy bands. II. The degenerate band case. Proc. R. Soc. London A, 277:237-259, 1964.

[51] Hubbard, J. Electron correlations in narrow energy bands. III. An improved solution. Proc. R. Soc. London A, 281:401-419, 1964.

[52] Hubbard, J. Electron correlations in narrow energy bands. IV. The atomic representation. Proc. R. Soc. London A, 285:542-558, 1965.

[53] Hubbard, J. Electron correlations in narrow energy bands. V. A perturbation expansion about the atomic limit. Proc. R. Soc. London A, 296:82-99, 1967.

[54] Hubbard, J. Electron correlations in narrow energy bands. VI. The connexion with many-body perturbation theory. Proc. R. Soc. London A, 296:100-112, 1967.

[55] Anisimov, V. I.; Gunnarsson, O. Density-functional calculation of effective Coulomb interactions in metals. Phys. Rev. B, 43:7570-7574, 1991.

[56] Anisimov, V. I.; Gunnarsson, O. Band theory and Mott insulators: Hubbard U instead of Stoner I. Phys. Rev. B, 44:943-954, 1991.

[57] Anisimov, V. I.; Solovyev, I. V.; Korotin, M. A. Czyżyk, M. T.; Sawatzky, G. A. Densityfunctional theory and NiO photoemission spectra. Phys. Rev. B, 48:16929-16934, 1993.

[58] Solovyev, I. V.; Dederichs, P. H.; Anisimov, V. I. Corrected atomic limit in the localdensity approximation and the electronic structure of $d$ impurities in Rb. Phys. Rev. B, 50:16861-16871, 1994.

[59] Anisimov, V. I.; Aryasetiawan, F.; Lichtenstein, A. I. First-principles calculations of the electronic structure and spectra of strongly correlated systems: the LDA $+\mathrm{U}$ method. J. Phys.: Condens. Matter, 48:16929-16934, 1997.

[60] Dudarev, S. L.; Botton, G. A.; Savrasov, S. Y.; Humphreys, C. J.; Sutton, A. P. Electron-energy-loss spectra and the structural stability of nickel oxide: An LSDA+U study. Phys. Rev. B, 57:1505-1509, 1998.

[61] Heyd, J.; Scuseria, G. E.; Ernzerhof, M. Hybrid functionals based on a screened Coulomb potential. J. Chem. Phys., 118:8207-8215, 2003.

[62] Adamo, C.; Barone, V. Toward reliable density functional methods without adjustable parameters: The PBE0 model. J. Chem. Phys., 110:6158-6170, 1999. 
[63] Bloch, F. Über die Quantenmechanik der Elektronen in Kristallgittern. Z. Physik, 52:555-600, 1929.

[64] Rostgaard, C. The Projector Augmented-wave Method. ArXiv e-prints, page arXiv:0910.1921, 2009.

[65] Blöchl, P. E.; Först, C. J.; Schimpl, J. Projector augmented wave method: ab initio molecular dynamics with full wave functions. Bull. Mater. Sci., 26:33-41, 2003.

[66] Blöchl, P. E. Projector augmented-wave method. Phys. Rev. B, 50:17953-17979, 1994.

[67] Herring, C. A new method for calculating wave functions in crystals. Phys. Rev., 57:1169-1177, 1940.

[68] Phillips, J. C.; Kleinman, L. New method for calculating wave functions in crystals and molecules. Phys. Rev., 116:287-294, 1959.

[69] Antončík, E. A new formulation of the method of nearly free electrons. Czech. J. Phys., 4:439-451, 1954.

[70] Antončík, E. Approximate formulation of the orthogonalized plane-wave method. J. Phys. Chem. Solids, 10:314-320, 1959.

[71] Kresse, G.; Joubert, D. From ultrasoft pseudopotentials to the projector augmentedwave method. Phys. Rev. B, 59:1758-1775, 1999.

[72] Kresse, G.; Hafner, J. Ab initio molecular dynamics for liquid metals. Phys. Rev. B, 47:558-561, 1993.

[73] Kresse, G.; Hafner, J. Ab initio molecular-dynamics simulation of the liquid-metal amorphous-semiconductor transition in germanium. Phys. Rev. B, 49:14251-14269, 1994.

[74] Kresse, G.; Furthmüller, J. Efficiency of ab-initio total energy calculations for metals and semiconductors using a plane-wave basis set. Comput. Mater. Sci., 6:15-50, 1996.

[75] Kresse, G.; Furthmüller, J. Efficient iterative schemes for $a b$ initio total-energy calculations using a plane-wave basis set. Phys. Rev. B, 54:11169-11186, 1996.

[76] Momma, K.; Izumi, F. VESTA 3 for three-dimensional visualization of crystal, volumetric and morphology data. J. Appl. Crystallogr., 44:1272-1276, 2011.

[77] Chung, E. M. L.; McIntyre, G. J.; Paul, D. McK.; Balakrishnan, G.; Lees, M. R. Oxygen moment formation and canting in $\mathrm{Li}_{2} \mathrm{CuO}_{2}$. Phys. Rev. B, 68:144410, 2003. 
[78] Berger, R.; Tergenius, L.-E. Room temperature synthesis and structural characterization of monoclinic $\mathrm{LiCuO}_{2}$ by X-ray and neutron diffraction. J. Alloys Compd., 203:203-207, 1994.

[79] Xiang, H. J.; Lee, C.; Whangbo, M. H. Absence of a spiral magnetic order in $\mathrm{Li}_{2} \mathrm{CuO}_{2}$ containing one-dimensional $\mathrm{CuO}_{2}$ ribbon chains. Phys. Rev. B, 76:220411, 2007.

[80] Xiao, P.; Deng, Z. Q.; Manthiram, A.; Henkelman, G. Calculations of oxygen stability in lithium-rich layered cathodes. J. Phys. Chem. C, 116:23201-23204, 2012.

[81] Chevrier, V. L.; Ong, S. P.; Armiento, R.; Chan, M. K. Y.; Ceder, G. Hybrid density functional calculations of redox potentials and formation energies of transition metal compounds. Phys. Rev. B, 82:075122, 2010.

[82] Hinuma, Y.; Meng, Y. S.; Kang, K.; Ceder, G. Phase transitions in the $\mathrm{LiNi}_{0.5} \mathrm{Mn}_{0.5} \mathrm{O}_{2}$ system with temperature. Chem. Mater., 19:1790-1800, 2007.

[83] Morgan, B. J.; Watson, G. W. A DFT+U description of oxygen vacancies at the $\mathrm{TiO}_{2}$ rutile (110) surface. Surf. Sci., 601:5034-5041, 2007.

[84] Yang, W.; Parr, R. G. Hardness, softness, and the fukui function in the electronic theory of metals and catalysis. Proc. Natl. Acad. Sci. U.S.A., 82:6723-6726, 1985.

[85] Cohen, M. H.; Ganduglia-Pirovano, M. V.; Kudrnovský, J. Electronic and nuclear chemical reactivity. J. Chem. Phys., 101:8988-8997, 1994.

[86] Geerlings, P.; De Proft, F.; Langenaeker, W. Conceptual density functional theory. Chem. Rev., 103:1793-1874, 2003.

[87] Cohen, M. H.; Ganduglia-Pirovano, M. V.; Kudrnovský, J. Reactivity kernels, the normal modes of chemical reactivity, and the hardness and softness spectra. $J$. Chem. Phys., 103:3543-3551, 1995.

[88] Cohen, M. H.; Ganduglia-Pirovano, M. V.; Kudrnovský, J. Orbital symmetry, reactivity, and transition metal surface chemistry. Phys. Rev. Lett., 72:3222-3225, 1994.

[89] Wilke, S.; Cohen, M. H.; Scheffler, M. Local isoelectronic reactivity of solid surfaces. Phys. Rev. Lett., 77:1560-1563, 1996.

[90] Brommer, K. D.; Galván, M.; Dal Pino, A.; Joannopoulos, J. D. Theory of adsorption of atoms and molecules on $\operatorname{Si}(111)-(7 \times 7)$. Surf. Sci., 314:57-70, 1994.

[91] Pino-Rios, R.; Yañez, O.; Inostroza, D.; Ruiz, L.; Cardenas, C.; Fuentealba, P.; Tiznado, W. Proposal of a simple and effective local reactivity descriptor through a topological analysis of an orbital-weighted fukui function. J. Comput. Chem., 38:481-488, 2017. 
[92] Perdew, J. P.; Ruzsinszky, A.; Csonka, G. I.; Vydrov, O. A.;. Scuseria, G. E.; Constantin, L. A.; Zhou, X.; Burke, K. Restoring the Density-Gradient expansion for exchange in solids and surfaces. Phys. Rev. Lett., 100:136406, 2008.

[93] Sun, J.; Ruzsinszky, A.; Perdew, J. P. Strongly constrained and appropriately normed semilocal density functional. Phys. Rev. Lett., 115:036402, 2015.

[94] Aydinol, M. K.; Kohan, A. F.; Ceder, G. Ab initio calculation of the intercalation voltage of lithium-transition-metal oxide electrodes for rechargeable batteries. $J$. Power Sources, 68:664-668, 1997.

[95] Aydinol, M. K.; Ceder, G. First-Principles prediction of insertion potentials in Li-Mn oxides for secondary Li batteries. J. Electrochem. Soc., 144:3832-3835, 1997.

[96] Mackay, K. M.; Mackay, R. A.; Henderson, W. Introduction to Modern Inorganic Chemistry, 6a Edición. Taylor \& Francis, 2002.

[97] Bader, R. Atoms in molecules : a quantum theory. Clarendon Press, Oxford, 1994.

[98] Tang, W.; Sanville, E.; Henkelman, G. A grid-based bader analysis algorithm without lattice bias. J. Phys. Condens. Matter, 21:084204, 2009.

[99] Bethe, H. Theorie der beugung von elektronen an kristallen. Ann. Phys., 392(17):55-129, 1928.

[100] Müller, U. Inorganic Structural Chemistry, Second Edition. John Wiley \& Sons, Ltd., 2006.

[101] Kotz, J. C.; Treichel, P. M.; Townsend, J. R. Chemistry \& Chemical Reactivity. Thomson Brooks/Cole, 2009.

[102] Sapiña, F.; Rodríguez-Carvajal, J.; Sanchis, M. J.; Ibáñez, R.; Beltrán, A.; Beltrán, D. Crystal and magnetic structure of $\mathrm{Li}_{2} \mathrm{CuO}_{2}$. Solid State Commun., 74:779-784, 1990.

[103] Yang, W.; Mortier, W. J. The use of global and local molecular parameters for the analysis of the gas-phase basicity of amines. J. Am. Chem. Soc., 108:5708-5711, 1986.

[104] Bratsch, S. G. Standard electrode potentials and temperature coefficients in water at 298.15 K. J. Phys. Chem. Ref. Data, 18:1-21, 1989.

[105] Bosecker, K. Bioleaching: metal solubilization by microorganisms. FEMS Microbiol. Rev., 20:591-604, 1997.

[106] Smith, R. W.; Misra, M. Mineral Bioprocessing. TMS, 1991. 
[107] Nowaczyk, K.; Domka, F. Oxidation of pyrite and marcasite by Thiobacillus ferrooxidans bacteria. Pol. J. Environ. Stud., 9, 2000.

[108] Li, Y.; Chen, J.; Zhao, C. Influence of external electric field on the electronic structure and optical properties of pyrite. RSC Adv., 7:56676-56681, 2017.

[109] Dos Santos, E. C.; Lourenço, M. P. Pettersson, L. G. M.; Duarte, H. A. Stability, structure, and electronic properties of the pyrite/arsenopyrite solid-solid interface-a DFT study. J. Phys. Chem. C, 121:8042-8051, 2017.

[110] Conejeros, S.; Alemany, P.; Llunell, M.; Moreira, I. de P. R.; Sánchez, V.; Llanos, J. Electronic structure and magnetic properties of $\mathrm{CuFeS}_{2}$. Inorg. Chem., 54:48404849, 2015.

[111] Dutrizac, J. E. The dissolution of chalcopyrite in ferric sulfate and ferric chloride media. Metall. Mater. Trans. B, 12:371-378, 1981.

[112] Mejía, E. R.; Ospina, J. D.; Márquez, M. A.; Morales, A. L. Oxidation of chalcopyrite $\left(\mathrm{CuFeS}_{2}\right)$ by Acidithiobacillus ferrooxidans and a mixed culture of Acidithiobacillus ferrooxidans and Acidithiobacillus thiooxidans like bacterium in shake flasks. In Biohydrometallurgy 2009, volume 71 of Advanced Materials Research, pages 385388. Trans. Tech. Publications, 2009.

[113] Lara, R. H.; Vazquez-Arenas, J.; Ramos-Sanchez, G.; Galvan, M.; Lartundo-Rojas, L. Experimental and theoretical analysis accounting for differences of pyrite and chalcopyrite oxidative behaviors for prospective environmental and bioleaching applications. J. Phys. Chem. C, 119:18364-18379, 2015.

[114] Engin, T. E.; Powell, A. V.; Hull, S. A high temperature diffraction-resistance study of chalcopyrite, $\mathrm{CuFeS}_{2}$. J. Solid State Chem., 184(8):2272-2277, 2011.

[115] Lara, R. H.; Velázquez, L. J.; Vazquez-Arenas, J.; Mallet, M.; Dossot, M.; Labastida, I.; Sosa-Rodríguez, F. S.; Espinosa-Cristóbal, L. F.; Escobedo-Bretado, M. A.; Cruz, R. Arsenopyrite weathering under conditions of simulated calcareous soil. Environ. Sci. Pollut. Res., 23:3681-3706, 2016.

[116] Ramírez-Aldaba, H.; Vazquez-Arenas, J.; Sosa-Rodríguez, F. S.; Valdez-Pérez, D.; Ruiz-Baca, E.; García-Meza, J. V.; Trejo-Córdova, G.; Lara, R. H. Assessment of biofilm changes and concentration-depth profiles during arsenopyrite oxidation by Acidithiobacillus thiooxidans. Environ. Sci. Pollut. Res., 24:20082-20092, 2017.

[117] Tauson, V. L.; Kravtsova, R. G.; Grebenshchikova, V. I.; Lustenberg, E. E.; Lipko, S. V. Surface typochemistry of hydrothermal pyrite: Electron spectroscopic and scanning probe microscopic data. II. Natural pyrite. Geochem. Int., 47:231, 2009. 
[118] Schippers, A.; Sand, W. Bacterial leaching of metal sulfides proceeds by two indirect mechanisms via thiosulfate or via polysulfides and sulfur. Appl. Environ. Microbiol., 65:319-321, 1999.

[119] Todd, E. C.; Sherman, D. M.; Purton, J. A. Surface oxidation of pyrite under ambient atmospheric and aqueous ( $\mathrm{pH}=2$ to 10$)$ conditions: electronic structure and mineralogy from X-ray absorption spectroscopy. Geochim. Cosmochim. Acta, 67:881 - 893, 2003. Advances in Oxide and Sulfide Mineral Surface Chemistry.

[120] Chandra, A. P.; Gerson, A. R. The mechanisms of pyrite oxidation and leaching: A fundamental perspective. Surf. Sci. Rep., 65:293-315, 2010.

[121] Wang, H.; Bigham, J. M.; Tuovinen, O. H. Oxidation of marcasite and pyrite by iron-oxidizing bacteria and archaea. Hydrometallurgy, 88:127-131, 2007.

[122] Akcil, A.; Koldas, S. Acid Mine Drainage (AMD): causes, treatment and case studies. J. Clean. Prod., 14:1139-1145, 2006. Improving Environmental, Economic and Ethical Performance in the Mining Industry. Part 2. Life cycle and process analysis and technical issues.

[123] Peppas, A.; Komnitsas, K.; Halikia, I. Use of organic covers for acid mine drainage control. Miner. Eng., 13:563-574, 2000.

[124] Sun, R.; Chan, M. K. Y.; Ceder, G. First-principles electronic structure and relative stability of pyrite and marcasite: Implications for photovoltaic performance. Phys. Rev. B, 83:235311, 2011. 
ESTUDIO TEORICO DE CUPRATOS DE LITIO COMO POSIBLES MATERIALES CATÓDICOS PARA BATERIAS.

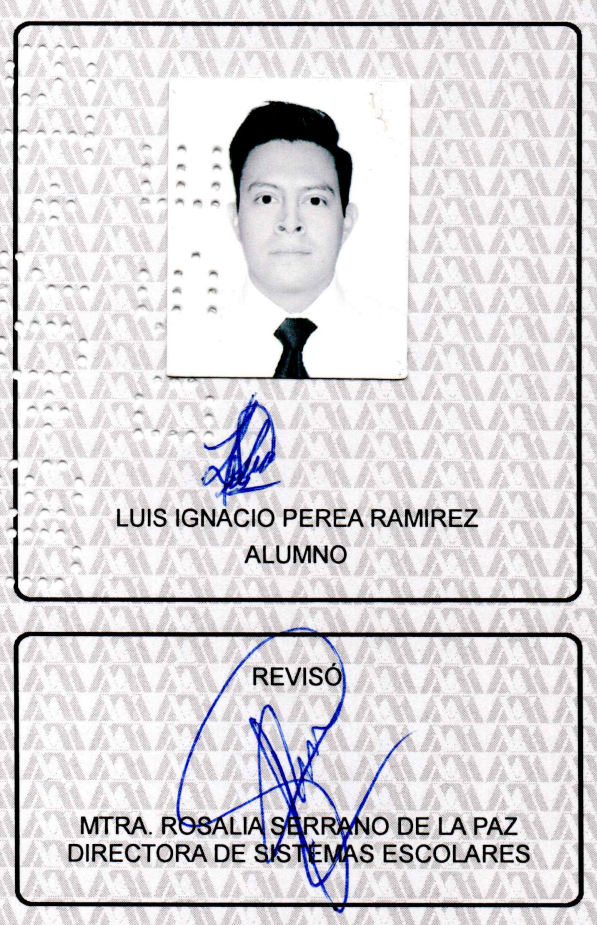

DIRECTOR DE LA DIVISIÓN DE CBI

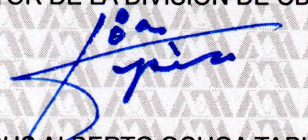

DR. JESUSALBERTO OCHOA TAPIA

\section{suscritos miembros del jurado: \\ DR. IGNACIO GONZALEZ MARTINEZ \\ DRA. ANNIA GALANO JIMENEZ \\ DR. MARIO FIDEL GARCIA SANCHEZ \\ DR. JOAQUIN BARROSO FLORES \\ DR. JOEL IRETA MORENO}

En la Ciudad de México, se presentaron a las 11:00 horas del día 1 del mes de julio del año 2019 en la Unidad Iztapalapa de la Universidad Autónoma Metropolitana, los

Bajo la Presidencia del primero y con carácter de Secretario el último, se reunieron a la presentación de la Disertación pública cuya denominación aparece al margen, para la obtención del grado de:

DOCTOR EN CIENCIAS (QUIMICA)

DE: LUIS IGNACIO PEREA RAMIREZ

y de acuerdo con el artículo 78 fracción IV del Reglamento de Estudios Superiores de la Universidad Autónoma Metropolitana, los miembros del jurado resolvieron:

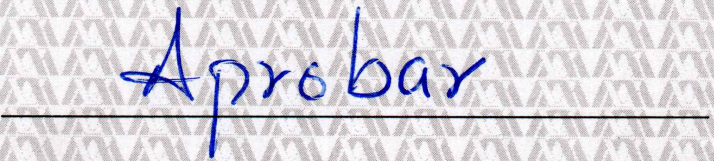

Acto continuo, el presidente del jurado comunicó al interesado el resultado de la evaluación y, en caso aprobatorio, le fue tomada la protesta.
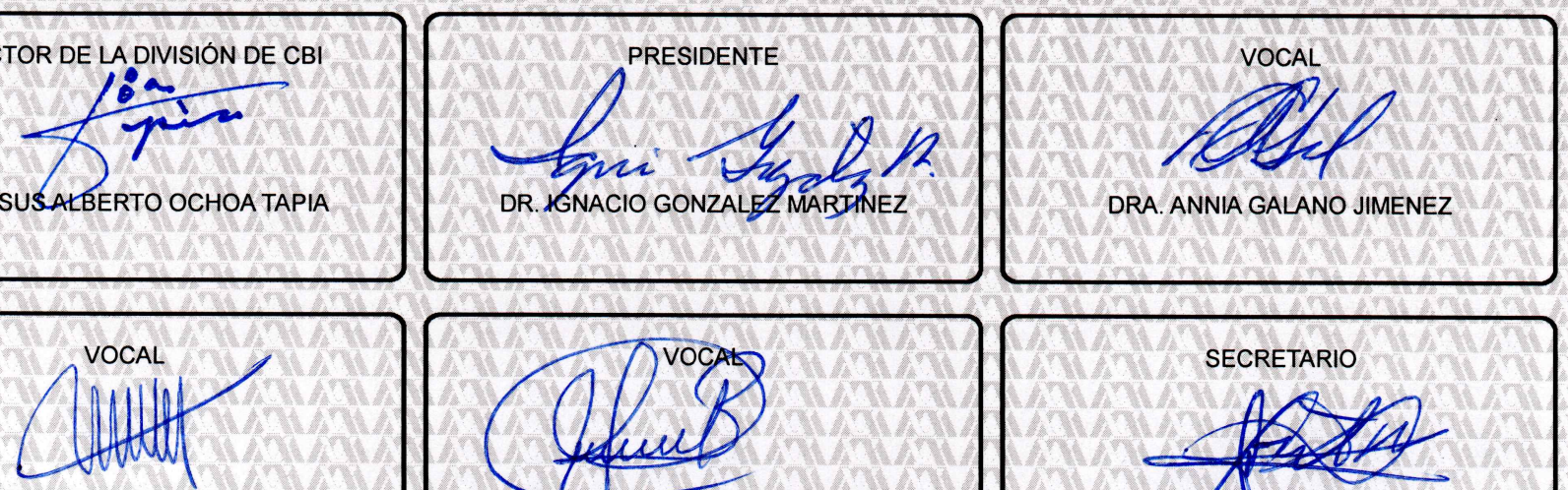

DR. MARIO FIDEL GARCIA SANCHEZ
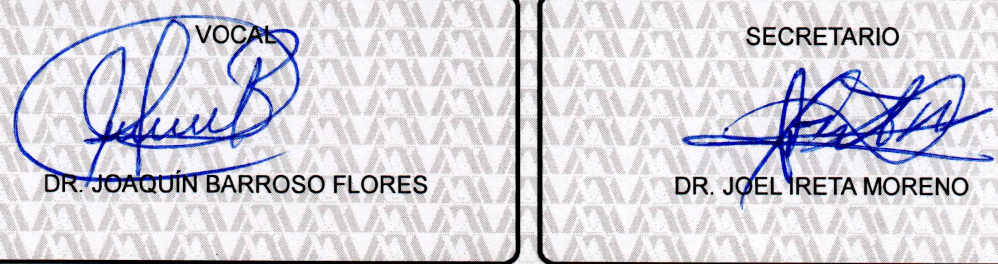